\title{
On chiral extrapolations of charmed meson masses and coupled-channel reaction dynamics
}

\author{
Xiao-Yu Guo, ${ }^{1}$ Yonggoo Heo, ${ }^{1}$ and Matthias F. M. Lutz ${ }^{1,2}$ \\ ${ }^{1}$ GSI Helmholtzzentrum für Schwerionenforschung GmbH, Planckstraße 1, 64291 Darmstadt, Germany \\ ${ }^{2}$ Technische Universität Darmstadt, D-64289 Darmstadt, Germany
}

(Received 30 January 2018; published 18 July 2018)

\begin{abstract}
We perform an analysis of QCD lattice data on charmed-meson masses. The quark-mass dependence of the data set is used to gain information on the size of counterterms of the chiral Lagrangian formulated with opencharm states with $J^{P}=0^{-}$and $J^{P}=1^{-}$quantum numbers. Of particular interest are those counterterms that are active in the exotic flavor sextet channel. A chiral expansion scheme in which physical masses enter the extrapolation formulas is developed and applied to the lattice data set. Good convergence properties are demonstrated, and an accurate reproduction of the lattice data based on ensembles of PACS-CS, MILC, ETMC, and HSC with pion and kaon masses smaller than $600 \mathrm{MeV}$ is achieved. It is argued that a unique set of lowenergy parameters is obtainable only if additional information from HSC on some scattering observables is included in our global fits. The elastic and inelastic s-wave $\pi D$ and $\eta D$ scattering as considered by HSC is reproduced faithfully. Based on such low-energy parameters, we predict 15 phase shifts and inelasticities at physical quark masses but also for an additional HSC ensemble at smaller pion mass. In addition, we find a clear signal for a member of the exotic flavor sextet states in the $\eta D$ channel, below the $\bar{K} D_{s}$ threshold. For the isospin-violating strong decay width of the $D_{s 0}^{*}(2317)$, we obtain the range $(104-116) \mathrm{keV}$.
\end{abstract}

DOI: 10.1103/PhysRevD.98.014510

\section{INTRODUCTION}

Systems with one heavy and one light quark play a particularly important role in the spectroscopy of QCD [1-4]. Two distinct approximate symmetries characterize the spectrum of open-charm mesons. While in the limit of an infinitely heavy charm quark the heavy-quark spin symmetry arises, the opposite limit with vanishing masses for the up, down, and strange quark mass leads to the flavor $\mathrm{SU}(3)$ chiral symmetry. The approximate chiral symmetry of the up, down, and strange quarks guides the construction of effective field theory approaches based on the chiral Lagrangian. There are two complementary approaches feasible. Either one may construct an effective chiral Lagrangian formulated in terms of heavy-quark multiplet fields $[1,2,5]$ or one may start with an effective chiral Lagrangian with fully relativistic fields, wherein the low-energy constants are correlated by constraints from the heavy-quark spin symmetry [6,7]. The former approach may be more economic in applications in which the coupled-channel

Published by the American Physical Society under the terms of the Creative Commons Attribution 4.0 International license. Further distribution of this work must maintain attribution to the author(s) and the published article's title, journal citation, and DOI. Funded by SCOAP ${ }^{3}$. unitarity constraint is implemented by means of partial summation techniques [8-13].

A striking prediction of the leading-order chiral interaction of the Goldstone bosons with the $D$ mesons with either $J^{P}=0^{-}$or $J^{P}=1^{-}$is an attractive short-range force in the exotic flavor sextet channel $[6,8,9]$. The strength of this interaction is somewhat reduced as compared to a corresponding force in the conventional flavor triplet channel that can be successfully used to describe the lowest scalar and axial-vector states in the open-charm meson spectrum $[6,8,9,11-16]$. Whether the chiral force in the flavor sextet sector leads to the formation of exotic open-charm meson states is an open issue. The possible existence of such an exotic flavor sextet multiplet of states depends on the precise form of chiral correction terms [6,9].

In this work, we wish to study the size of such chiral counterterms. First rough studies [6,9] suffer from limited empirical constraints. Additional information from first QCD lattice simulation on a set of s-wave scattering lengths was used in a series of later works [10-13]. Results that in part show unnaturally large counterterms and/or illustrate some residual dependence on how to set up the coupledchannel computation are obtained. Here, we follow a different path and try to use the recent data set on the quark-mass dependence of the $D$-meson ground-state masses [17-23]. This dynamics is driven in part by the counterterms that also have a significant impact on the open-charm coupled-channel systems as discussed above. 
One may hope to obtain results that are less model dependent in this case.

However, it is well known that chiral perturbation theory formulated with three light flavors does not always show a convincing convergence pattern [24-31]. How is this for the case at hand? Only a few studies are available in which this issue is addressed for open-charm meson systems. In a recent work, the authors presented a novel chiral extrapolation scheme for the quark-mass dependence of the baryon octet and decuplet states that is formulated in terms of physical masses [32-35]. It is the purpose of our study to adapt this scheme to the open-charm sector of QCD and apply it to the available lattice data set. This requires in particular considering the $D$ mesons with $J^{P}=0^{-}$and $J^{P}=1^{-}$quantum numbers on equal footing. For a given set of low-energy constants, each set of the four $D$-meson masses has to be determined numerically as a solution of a nonlinear system.

The work is organized as follows. In Sec. II, the part of the chiral Lagrangian that is relevant here is recalled. It follows a section in which the one-loop contributions to the $D$-meson masses are derived in a finite box. We do not consider discretization effects in our study. In Secs. IV and
$\mathrm{V}$, power counting in the presence of physical masses is discussed. The application to available lattice data sets is presented in Secs. VI-VIII. Lattice data taken on ensembles of PACS-CS, MILC, ETMC, and HSC are considered. The results for the $D$-meson masses are considered in Sec. VI. The various sets of low-energy parameters are collected in Sec. VI and Sec. VII. Our results for the s-wave scattering lengths can be found in Sec. VII. In Sec. VIII, we present our predictions for phase shifts and inelasticities based on the parameter sets of Sec. VII as obtained form the considered lattice data. In Sec. IX our values for the isospin violating decay width of the $D_{s 0}^{*}(2317)$ are presented. With a summary and outlook, the paper is closed.

\section{CHIRAL LAGRANGIAN WITH OPEN-CHARM MESON FIELDS}

We recall the chiral Lagrangian formulated in the presence of two antitriplets of $D$ mesons with $J^{P}=0^{-}$ and $J^{P}=1^{-}$quantum numbers $[1,2]$. In the relativistic version, the Lagrangian was developed in Refs. $[6,8,9]$. The kinetic terms read

$$
\begin{aligned}
\mathcal{L}_{\text {kin }}= & \left(\hat{\partial}_{\mu} D\right)\left(\hat{\partial}^{\mu} \bar{D}\right)-M^{2} D \bar{D}-\left(\hat{\partial}_{\mu} D^{\mu \alpha}\right)\left(\hat{\partial}^{\nu} \bar{D}_{\nu \alpha}\right)+\frac{1}{2}(M+\Delta)^{2} D^{\mu \alpha} \bar{D}_{\mu \alpha} \\
& -f^{2} \operatorname{tr}\left\{U_{\mu} U^{\mu}\right\}+\frac{1}{2} f^{2} \operatorname{tr}\left\{\chi_{+}\right\},
\end{aligned}
$$

where

$$
\begin{aligned}
U_{\mu} & =\frac{1}{2} e^{-i \frac{\Phi}{2 f}}\left(\partial_{\mu} e^{i \frac{\Phi}{f}}\right) e^{-i \frac{\Phi}{2 f}}, \quad \Gamma_{\mu}=\frac{1}{2} e^{-i \frac{\Phi}{2 f}} \partial_{\mu} e^{+i \frac{\Phi}{2 f}}+\frac{1}{2} e^{+i \frac{\Phi}{2 f}} \partial_{\mu} e^{-i \frac{\Phi}{2 f}}, \\
\chi_{ \pm} & =\frac{1}{2}\left(e^{+i \frac{\Phi}{2 f}} \chi_{0} e^{+i \frac{\Phi}{2 f}} \pm e^{-i \frac{\Phi}{2 f}} \chi_{0} e^{-i \frac{\Phi}{2 f}}\right), \quad \chi_{0}=2 B_{0} \operatorname{diag}\left(m_{u}, m_{d}, m_{s}\right), \\
\hat{\partial}_{\mu} \bar{D} & =\partial_{\mu} \bar{D}+\Gamma_{\mu} \bar{D}, \quad \hat{\partial}_{\mu} D=\partial_{\mu} D-D \Gamma_{\mu} .
\end{aligned}
$$

Following Ref. [6], we represent the $1^{-}$field in terms of an antisymmetric tensor field $D_{\mu \nu}$. The covariant derivative $\hat{\partial}_{\mu}$ involves the chiral connection $\Gamma_{\mu}$, the quark masses enter via the symmetry-breaking fields $\chi_{ \pm}$, and the octet of the Goldstone boson fields is encoded into the $3 \times 3$ matrix $\Phi$. The parameter $f$ is the chiral limit value of the pion-decay constant. Finally, given our particular renormalization scheme, the parameters $M$ and $M+\Delta$ give the masses of the $D$ and $D^{*}$ mesons in that limit with $m_{u}=m_{d}=m_{s}=0$.

We continue with first-order interaction terms

$$
\begin{aligned}
\mathcal{L}^{(1)}= & 2 g_{P}\left\{D_{\mu \nu} U^{\mu}\left(\hat{\partial}^{\nu} \bar{D}\right)-\left(\hat{\partial}^{\nu} D\right) U^{\mu} \bar{D}_{\mu \nu}\right\} \\
& \left.-\frac{i}{2} \tilde{g}_{P} \epsilon^{\mu \nu \alpha \beta}\left\{D_{\mu \nu} U_{\alpha}\left(\hat{\partial}^{\tau} \bar{D}_{\tau \beta}\right)+\left(\hat{\partial}^{\tau} D_{\tau \beta}\right) U_{\alpha} \bar{D}_{\mu \nu}\right)\right\},
\end{aligned}
$$

which upon an expansion in powers of the Goldstone boson fields provide the three-point coupling constants of the
Goldstone bosons to the $D$ mesons. While the decay of the charged $D^{*}$ mesons [6] implies

$$
\left|g_{P}\right|=0.57 \pm 0.07
$$

the parameter $\tilde{g}_{P}$ in (3) cannot be extracted from empirical data directly. The size of $\tilde{g}_{P}$ can be estimated using the heavy-quark symmetry of QCD $[1,2]$. At leading order, one expects $\tilde{g}_{P}=g_{P}$.

Second-order terms of the chiral Lagrangian were first studied in Refs. [6,9], in which the focus was on counterterms relevant for s-wave scattering of Goldstone bosons with the $D$ mesons. A list of eight terms with dimensionless parameters $c_{i}$ and $\tilde{c}_{i}$ was identified. This list was extended by further terms relevant for $\mathrm{p}$-wave scattering in Ref. [36]. A complete collection of relevant terms is 


$$
\begin{aligned}
\mathcal{L}^{(2)}= & -\left(4 c_{0}-2 c_{1}\right) D \bar{D} \operatorname{tr} \chi_{+}-2 c_{1} D \chi_{+} \bar{D}+4\left(2 c_{2}+c_{3}\right) D \bar{D} \operatorname{tr}\left(U_{\mu} U^{\mu \dagger}\right)-4 c_{3} D U_{\mu} U^{\mu \dagger} \bar{D} \\
& +\frac{1}{M^{2}}\left(4 c_{4}+2 c_{5}\right)\left(\hat{\partial}_{\mu} D\right)\left(\hat{\partial}_{\nu} \bar{D}\right) \operatorname{tr}\left[U^{\mu}, U^{\nu \dagger}\right]_{+}-\frac{1}{M^{2}} 2 c_{5}\left(\hat{\partial}_{\mu} D\right)\left[U^{\mu}, U^{\nu \dagger}\right]_{+}\left(\hat{\partial}_{\nu} \bar{D}\right) \\
& +i c_{6} \epsilon^{\mu \nu \rho \sigma}\left(D\left[U_{\mu}, U_{\nu}^{\dagger}\right]_{-} \bar{D}_{\rho \sigma}-D_{\rho \sigma}\left[U_{\nu}^{\dagger}, U_{\mu}\right]_{-} \bar{D}\right)+\left(2 \tilde{c}_{0}-\tilde{c}_{1}\right) D^{\mu \nu} \bar{D}_{\mu \nu} \operatorname{tr} \chi_{+}+\tilde{c}_{1} D^{\mu \nu} \chi_{+} \bar{D}_{\mu \nu} \\
& -\left(4 \tilde{c}_{2}+2 \tilde{c}_{3}\right) D^{\alpha \beta} \bar{D}_{\alpha \beta} \operatorname{tr}\left(U_{\mu} U^{\mu \dagger}\right)+2 \tilde{c}_{3} D^{\alpha \beta} U_{\mu} U^{\mu \dagger} \bar{D}_{\alpha \beta} \\
& -\frac{1}{(M+\Delta)^{2}}\left(2 \tilde{c}_{4}+\tilde{c}_{5}\right)\left(\hat{\partial}_{\mu} D^{\alpha \beta}\right)\left(\hat{\partial}_{\nu} \bar{D}_{\alpha \beta}\right) \operatorname{tr}\left[U^{\mu}, U^{\nu \dagger}\right]_{+} \\
& +\frac{1}{(M+\Delta)^{2}} \tilde{c}_{5}\left(\hat{\partial}_{\mu} D^{\alpha \beta}\right)\left[U^{\mu}, U^{\nu \dagger}\right]_{+}\left(\hat{\partial}_{\nu} \bar{D}_{\alpha \beta}\right)-4 \tilde{c}_{6} D^{\mu \alpha}\left[U_{\mu}, U^{\nu \dagger}\right]_{-} \bar{D}_{\nu \alpha},
\end{aligned}
$$

where the parameters $M$ and $M+\Delta$ are the $D$ and $D^{*}$ meson masses as evaluated at $m_{u}=m_{d}=m_{s}=0$. In the limit of a very large charm-quark mass, it follows that $M \rightarrow \infty$ but $\Delta \rightarrow 0$. All parameters $c_{i}$ and $\tilde{c}_{i}$ are expected to scale linearly in the parameter $M_{0}$. As illustrated in Ref. [6], it holds $\tilde{c}_{i}=c_{i}$ in the heavy quark-mass limit.

A first estimate of some parameters can be found in Ref. [6] based on large- $N_{c}$ arguments. Since at leading order in a $1 / N_{c}$ expansion single-flavor trace interactions are dominant, the corresponding couplings should go to zero in the $N_{c} \rightarrow \infty$ limit, suggesting

$$
\begin{aligned}
& c_{0} \simeq \frac{c_{1}}{2}, \quad c_{2} \simeq-\frac{c_{3}}{2}, \quad c_{4} \simeq-\frac{c_{5}}{2}, \\
& \tilde{c}_{0} \simeq \frac{\tilde{c}_{1}}{2}, \quad \tilde{c}_{2} \simeq-\frac{\tilde{c}_{3}}{2}, \quad \tilde{c}_{4} \simeq-\frac{\tilde{c}_{5}}{2} \text {. }
\end{aligned}
$$

In the combined heavy-quark and large- $N_{c}$ limit, we are left with four free parameters only, $c_{1}, c_{3}, c_{5}$, and $c_{6}$. For two of them, approximate ranges

$$
c_{1} \simeq 0.44-0.47, \quad c_{3}+c_{5} \simeq 1.0-1.4
$$

were obtained previously in Ref. [6]. While the parameter $c_{1}$ can be estimated from the $D$ meson masses, the parameter $c_{3}$ is constrained by the empirical $\pi D$-invariant mass spectrum [6,9]. A complementary estimate was explored in Ref. [10], in which the parameter $c_{3}+c_{5}$ was adjusted to first QCD lattice computations for s-wave scattering lengths of the Goldstone bosons with the $D$ mesons. It is remarkable that their range for $c_{3}+c_{5} \simeq 1$ is quite consistent with the earlier estimates [6,9] based on the empirical $\pi D$-invariant mass spectrum. The $c_{3}$ parameter is of crucial importance for the physics of two exotic sextets of $J^{P}=0^{+}$and $J^{P}=1^{+}$ resonances. Such multiplets are predicted by the leadingorder chiral Lagrangian (1), which entails in particular the Tomozawa-Weinberg coupled-channel interactions of the Goldstone bosons with the $D$ mesons [8]. The latter predicts weak attraction in the flavor sextet channel. If used as the driving term in a coupled-channel unitarization, exotic signals appear. A reliable estimate of the correction terms proportional to $c_{3}$ and $\tilde{c}_{3}$ is important in order to arrive at a detailed picture of this exotic sector of QCD [6,9].

We close this section with a first construction of the symmetry-breaking counterterms proportional to the product of two quark masses:

$$
\begin{aligned}
\mathcal{L}^{(4)}= & -d_{1} D \chi_{+}^{2} \bar{D}-d_{2} D \chi_{+} \bar{D} \operatorname{tr}\left(\chi_{+}\right)-d_{3} D \bar{D} \operatorname{tr}\left(\chi_{+}^{2}\right)-d_{4} D \bar{D}\left(\operatorname{tr} \chi_{+}\right)^{2} \\
& +\frac{1}{2} \tilde{d}_{1} D^{\mu \nu} \chi_{+}^{2} \bar{D}_{\mu \nu}+\frac{1}{2} \tilde{d}_{2} D^{\mu \nu} \chi_{+} \bar{D}_{\mu \nu} \operatorname{tr}\left(\chi_{+}\right)+\frac{1}{2} \tilde{d}_{3} D^{\mu \nu} \bar{D}_{\mu \nu} \operatorname{tr}\left(\chi_{+}^{2}\right)+\frac{1}{2} \tilde{d}_{4} D^{\mu \nu} \bar{D}_{\mu \nu}\left(\operatorname{tr} \chi_{+}\right)^{2}
\end{aligned}
$$

Such terms are relevant in the chiral extrapolation of the $D$-meson masses. For the pseudoscalar mesons, we provide the tree-level contributions to the polarization $\Pi_{H}^{(2)}$ and $\Pi_{H}^{(4-\chi)}$ of the $D$ and $D_{s}$ mesons. We use a convention with

$$
\begin{aligned}
M_{H \in\left[0^{-}\right]}^{2} & =M^{2}+\Pi_{H}^{(2)}+\Pi_{H}^{(4-\chi)}+\cdots, \quad M_{H \in\left[1^{-}\right]}^{2}=(M+\Delta)^{2}+\Pi_{H}^{(2)}+\Pi_{H}^{(4-\chi)}+\cdots, \\
\Pi_{D}^{(2)} & =2 B_{0}\left(4 c_{0}-2 c_{1}\right)\left(m_{s}+2 m\right)+4 B_{0} c_{1} m, \\
\Pi_{D}^{(4-\chi)} & =4 B_{0}^{2}\left(d_{1}+2 d_{2}+2 d_{3}+4 d_{4}\right) m^{2}+4 B_{0}^{2}\left(d_{3}+d_{4}\right) m_{s}^{2}+4 B_{0}^{2}\left(d_{2}+4 d_{4}\right) m m_{s}, \\
\Pi_{D_{s}}^{(2)} & =2 B_{0}\left(4 c_{0}-2 c_{1}\right)\left(m_{s}+2 m\right)+4 B_{0} c_{1} m_{s}, \\
\Pi_{D_{s}}^{(4-\chi)} & =4 B_{0}^{2}\left(2 d_{3}+4 d_{4}\right) m^{2}+4 B_{0}^{2}\left(d_{1}+d_{2}+d_{3}+d_{4}\right) m_{s}^{2}+4 B_{0}^{2}\left(2 d_{2}+4 d_{4}\right) m m_{s},
\end{aligned}
$$


where we consider the isospin limit with $m_{u}=m_{d}=m$. Analogous expressions hold for the vector mesons polarization $\Pi_{H \in\left[1^{-}\right]}^{(2)}$ and $\Pi_{H \in\left[1^{-}\right]}^{(4-\chi)}$, where the replacements $c_{i} \rightarrow \tilde{c}_{i}$ and $d_{i} \rightarrow \tilde{d}_{i}$ are to be applied to (9). With $\tilde{c}_{i}=c_{i}$ and $\tilde{d}_{i}=$ $d_{i}$ and $\Delta \rightarrow 0$, the heavy-quark spin symmetry is recovered exactly.

We need to mention a technical issue. The propagator $S_{\mu \nu}^{\alpha \beta}(p)$ of our $1^{-}$fields involves four Lorentz indices, which are pairwise antisymmetric. Interchanging either $\alpha \leftrightarrow \beta$ or $\mu \leftrightarrow \nu$ generates a change in sign. A mass renormalization from a loop contribution arises from a particular projection $\Pi\left(p^{2}\right)$ of the polarization tensor $\Pi_{\alpha \beta}^{\mu \nu}(p)$ with

$$
\begin{aligned}
\Pi\left(p^{2}\right)= & \frac{-1}{(d-1) p^{2}}\left(g_{\mu \alpha} p_{\nu} p_{\beta}-g_{\mu \beta} p_{\nu} p_{\alpha}-g_{\nu \alpha} p_{\mu} p_{\beta}\right. \\
& \left.+g_{\nu \beta} p_{\mu} p_{\alpha}\right) \Pi^{\mu \nu, \alpha \beta}(p),
\end{aligned}
$$

where $d$ is the space-time dimension. This is the part that is used in (9) and will be used also in the following.

\section{ONE-LOOP MASS CORRECTIONS IN A FINITE BOX}

The chiral Lagrangian of Sec. II is used to compute the $D$-meson masses at the one-loop level. To prepare for a comparison of QCD lattice data, this computation is done in a finite box of volume $V$. A direct application of the relativistic chiral Lagrangian in the conventional $\overline{\mathrm{MS}}$ scheme does lead to a plethora of powercounting violating contributions. There are various ways to arrive at results that are consistent with the expectations of power-counting rules [32,37-39].

TABLE I. Coefficients $G_{Q R}^{(H)}$.

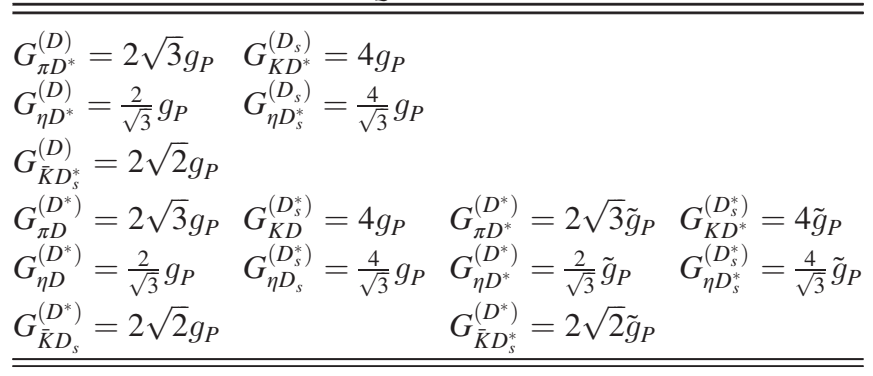

We follow here the $\chi$-MS approach developed previously for the chiral dynamics of baryons [32,40,41], which is based on the Passarino-Veltman reduction scheme [42]. Recently, this scheme was generalized for computations in a finite box [34]. This implies that all finite box effects are exclusively determined by the volume dependence of a set of universal scalar loop functions as discussed and presented in Ref. [34]. Our results will be expressed in terms of Clebsch coefficients $G_{Q R}^{(H)}$ and $G_{H Q}^{(\chi)}, G_{H Q}^{(S)}, G_{H Q}^{(V)}$, and a set of generic loop functions. While the index $H$ or $R$ runs over either the triplet of pseudoscalar or vector $D$ mesons, the index $Q$ runs over the octet of Goldstone bosons (see Tables I and II). In our case, there will be two tadpole integrals $\bar{I}_{Q}^{(0)}$ and $\bar{I}_{Q}^{(2)}$ from the Goldstone bosons and the scalar bubble-loop integral $\bar{I}_{Q R}$. In addition, there may be tadpole contributions $\bar{I}_{R}^{(n)}$ involving an intermediate $D$ meson. To render the power counting manifest, it suffices to supplement the Passarino-Veltman reduction scheme by a minimal and universal subtraction scheme [32]:

(i) Any tadpole integral involving a heavy particle is dropped.

(ii) The scalar bubble-loop integral requires a single subtraction.

The required loop functions have been used and detailed in previous works $[32,34]$ for finite box computations. For the readers' convenience, we recall the loop functions in the infinite box limit $[32,34]$ with

$$
\begin{aligned}
\bar{I}_{Q}^{(0)}= & \bar{I}_{Q}=\frac{m_{Q}^{2}}{(4 \pi)^{2}} \log \left(\frac{m_{Q}^{2}}{\mu^{2}}\right), \quad \bar{I}_{Q}^{(2)}=\frac{1}{4} m_{Q}^{2} \bar{I}_{Q}, \\
\bar{I}_{Q R}= & \frac{1}{16 \pi^{2}}\left\{\gamma_{R}^{H}-\left(\frac{1}{2}+\frac{m_{Q}^{2}-M_{R}^{2}}{2 M_{H}^{2}}\right) \log \left(\frac{m_{Q}^{2}}{M_{R}^{2}}\right)\right. \\
& +\frac{p_{Q R}}{M_{H}}\left(\log \left(1-\frac{M_{H}^{2}-2 p_{Q R} M_{H}}{m_{Q}^{2}+M_{R}^{2}}\right)\right. \\
& \left.\left.-\log \left(1-\frac{M_{H}^{2}+2 p_{Q R} M_{H}}{m_{Q}^{2}+M_{R}^{2}}\right)\right)\right\}, \\
\text { with } \quad p_{Q R}^{2}= & \frac{M_{H}^{2}}{4}-\frac{M_{R}^{2}+m_{Q}^{2}}{2}+\frac{\left(M_{R}^{2}-m_{Q}^{2}\right)^{2}}{4 M_{H}^{2}},
\end{aligned}
$$

TABLE II. Coefficients $G_{H Q}^{(\chi)}, G_{H Q}^{(S)}$, and $G_{H Q}^{(V)}$. The corresponding results for the $D^{*}$ and $D_{s}^{*}$ follow by the replacement $c_{i} \rightarrow \tilde{c}_{i}$ and $M \rightarrow M+\Delta$.

\begin{tabular}{ccccc}
\hline \hline$H$ & $Q$ & $G_{H Q}^{(\chi)} / B_{0}$ & $G_{H Q}^{(S)}$ & $M^{2} G_{H Q}^{(V)}$ \\
\hline$D$ & $\pi$ & $-48\left(2 c_{0}-c_{1}\right) m-24 c_{1} m$ & $24\left(2 c_{2}+c_{3}\right)-12 c_{3}$ & $24\left(2 c_{4}+c_{5}\right)-12 c_{5}$ \\
& $K$ & $-32\left(2 c_{0}-c_{1}\right)\left(m_{s}+m\right)-8 c_{1}\left(m_{s}+m\right)$ & $32\left(2 c_{2}+c_{3}\right)-8 c_{3}$ & $32\left(2 c_{4}+c_{5}\right)-8 c_{5}$ \\
& $\eta$ & $-\frac{16}{3}\left(2 c_{0}-c_{1}\right)\left(2 m_{s}+m\right)-\frac{8}{3} c_{1} m$ & $8\left(2 c_{2}+c_{3}\right)-\frac{4}{3} c_{3}$ & $8\left(2 c_{4}+c_{5}\right)-\frac{4}{3} c_{5}$ \\
$D_{s}$ & $\pi$ & $-48\left(2 c_{0}-c_{1}\right) m$ & $24\left(2 c_{2}+c_{3}\right)$ & $24\left(2 c_{4}+c_{5}\right)$ \\
& $K$ & $-32\left(2 c_{0}-c_{1}\right)\left(m_{s}+m\right)-16 c_{1}\left(m_{s}+m\right)$ & $32\left(2 c_{2}+c_{3}\right)-16 c_{3}$ & $32\left(2 c_{4}+c_{5}\right)-16 c_{5}$ \\
& $\eta$ & $-\frac{16}{3}\left(2 c_{0}-c_{1}\right)\left(2 m_{s}+m\right)-\frac{32}{3} c_{1} m_{s}$ & $8\left(2 c_{2}+c_{3}\right)-\frac{16}{3} c_{3}$ & $8\left(2 c_{4}+c_{5}\right)-\frac{16}{3} c_{5}$ \\
\hline \hline
\end{tabular}


where we note that in the infinite volume limit the two tadpole integrals $\bar{I}_{Q}^{(0)}$ and $\bar{I}_{Q}^{(2)}$ turn dependent and can no longer be discriminated in that case. The finite volume corrections for $\bar{I}_{Q}^{(0)}, \bar{I}_{Q}^{(2)}$, and $\bar{I}_{Q R}$ are detailed in Ref. [34].

We point at the presence of the additional subtraction term $\gamma_{R}^{H}=\gamma_{R}^{H}(M, \Delta)$ with

$$
\gamma_{R}^{H}=-\lim _{m, m_{s} \rightarrow 0} \frac{M_{R}^{2}-M_{H}^{2}}{M_{H}^{2}} \log \left|\frac{M_{R}^{2}-M_{H}^{2}}{M_{R}^{2}}\right|,
$$

as suggested recently in Ref. [34] in the analogous case of a baryon self-energy computation. The subtraction term depends on the chiral limit values $M$ and $M+\Delta$ of the $D$ and $D^{*}$ meson masses only. It was not yet imposed in earlier computations [32-34]. As was discussed in Ref. [34], the request of such a term comes from a study of the chiral regime with

$$
m_{Q} \ll \Delta \text { with } Q \in\{\pi, K, \eta\} .
$$

Within a counting scheme with $m_{Q} \sim \Delta \sim Q$, there is no need for any additional subtractions beyond the ones enforced by the $\chi$-MS approach. However, to arrive at consistent results for $m_{Q} \ll \Delta$, this subtraction is instrumental. While for $\Delta \sim m_{Q} \sim Q$ and $\gamma_{R}^{H}=0$ the scalar bubble scales with $\bar{I}_{Q R} \sim Q$ as expected from dimensional analysis, in the chiral regime, with $m_{Q} \ll \Delta$ and $m_{Q} \sim Q$, one would expect $\bar{I}_{Q R} \sim Q^{2} \sim m_{Q}^{2}$. This expectation turns true only for $\gamma_{R}^{H} \neq 0$ as chosen in (12).

We are now well prepared to collect all contributions to the $D$-meson self-energies at the one-loop level. Consider the bubble- and tadpole-loop contributions. The Passarino-Veltman reduction scheme in combination with the $\chi$-MS approach leads to the expressions

$$
\begin{aligned}
\Pi_{H \in\left[0^{-}\right]}^{\text {bubble }}= & \sum_{Q \in[8]} \sum_{R \in\left[1^{-}\right]}\left(\frac{G_{Q R}^{(H)}}{2 f}\right)^{2}\left\{-\frac{1}{4}\left(M_{H}^{2}-M_{R}^{2}+m_{Q}^{2}\right) \bar{I}_{Q}-M_{H}^{2} p_{Q R}^{2} \bar{I}_{Q R}\right\}, \\
\Pi_{H \in\left[0^{-}\right]}^{\text {tadpole }}= & \frac{1}{4 f^{2}} \sum_{Q \in[8]}\left(G_{\left.H Q^{(}\right)}^{(\chi)} \bar{I}_{Q}-G_{H Q^{(S)}} m_{Q^{2}} \bar{I}_{Q}-G_{H Q}^{(V)} M^{2} \bar{I}_{Q}^{(2)}\right), \\
\Pi_{H \in\left[1^{-}\right]}^{\text {bubble }}= & \sum_{Q \in[8]} \sum_{R \in\left[0^{-}\right]}\left(\frac{G_{Q R}^{(H)}}{2 f}\right)^{2}\left\{-\frac{1}{12}\left(M_{H}^{2}-M_{R}^{2}+m_{Q}^{2}\right) \bar{I}_{Q}-\frac{1}{3} M_{H}^{2} p_{Q R}^{2} \bar{I}_{Q R}\right\} \\
& +\sum_{Q \in[8]} \sum_{R \in\left[1^{-}\right]}\left(\frac{G_{Q R}^{(H)}}{2 f}\right)^{2}\left\{\frac{M_{H}^{2}+2 M_{R}^{2}}{12 M_{R}^{2}} \bar{I}_{Q}^{(2)}-\frac{\left(M_{H}^{2}+M_{R}^{2}\right)^{2}}{6 M_{R}^{2}} p_{Q R}^{2} \bar{I}_{Q R}\right. \\
& \left.-\left(\frac{\left(M_{H}^{2}-M_{R}^{2}\right)\left(M_{H}^{2}+M_{R}^{2}\right)^{2}}{24 M_{H}^{2} M_{R}^{2}}+\frac{M_{R}^{4}+6 M_{R}^{2} M_{H}^{2}-3 M_{H}^{4}}{24 M_{H}^{2} M_{R}^{2}} m_{Q}^{2}\right) \bar{I}_{Q}\right\}, \\
\Pi_{H \in\left[1^{-}\right]}^{\text {tadpole }}= & \frac{1}{4 f^{2}} \sum_{Q \in[8]}\left(G_{H Q}^{(\chi)} \bar{I}_{Q}-G_{H Q}^{(S)} m_{Q}^{2} \bar{I}_{Q}-G_{H Q}^{(V)}(M+\Delta)^{2} \bar{I}_{Q}^{(2)}\right),
\end{aligned}
$$

where the loop functions are expressed in terms of physical meson masses. The sums in (14) and (16) extend over intermediate Goldstone bosons $(Q)$ and pseudoscalar or vector $D$ mesons $(R)$ with either $R \in\left[0^{-}\right]$or $R \in\left[1^{-}\right]$. The Clebsch coefficients $G_{Q R}^{(H)}$ are specified in Table I. In the contributions from the tadpole diagrams, the sums in (15) and (17) extend over the intermediate Goldstone bosons $Q$. The coefficients $G_{H Q}^{(\chi)}, G_{H Q}^{(S)}$, and $G_{H Q}^{(V)}$ are listed in Table II.

The results (14), (16) deserve a detailed discussion. First, let us emphasize that a chiral expansion of the loop function as they are given confirms the leading chiral power as expected from dimensional counting rules. All powercounting violating contributions are subtracted, owing to the $\chi$-MS approach. Here, we adopted the conventional counting rules

$$
m_{Q} \sim Q \quad \text { and } \quad M_{1^{-}}-M_{0^{-}} \sim \Delta \sim Q,
$$

which is expected to be effective for $\Delta \sim m_{Q}$. Our results (14), (16) are model dependent, as there are various subtraction schemes available to obtain loop expressions that are compatible with dimensional counting rules. Most prominently, there is the infrared regularization of Becher and Leutwyler [38] and the minimal subtraction scheme proposed by Gegelia and Japaridze [39]. Following our previous work on the chiral extrapolation of the baryon masses, we will attempt to extract a model-independent part 
of such loop expressions. This goes in a few consecutive steps. The driving strategy behind this attempt is to keep the physical masses inside the loop function.

Consider first the terms that are proportional to the tadpole loop function $\bar{I}_{Q}$. There are two distinct classes of terms. The coefficient in front of any $\bar{I}_{Q}$ is proportional to either $m_{Q}^{2}$ or to $M_{H}^{2}-M_{R}^{2}$. The terms proportional to $m_{Q}^{2} \bar{I}_{Q}$ or also to $\bar{I}_{Q}^{(2)}$ in (14) and (16) have the same form as the corresponding structures in (15) and (17) and therefore renormalize the low-energy parameters $c_{n}$ and $\tilde{c}_{n}$ with

$c_{2}^{r}=c_{2}+\frac{1}{8} g_{P}^{2}, \quad c_{4}^{r}=c_{4}, \quad \tilde{c}_{4}^{r}=\tilde{c}_{4}-\frac{1}{8} \tilde{g}_{P}^{2}$,

$c_{3}^{r}=c_{3}-\frac{1}{4} g_{P}^{2}, \quad c_{5}^{r}=c_{5}, \quad \tilde{c}_{5}^{r}=\tilde{c}_{5}+\frac{1}{4} \tilde{g}_{P}^{2}$,

$\tilde{c}_{2}^{r}=\tilde{c}_{2}+\frac{1}{12} \tilde{g}_{P}^{2}+\frac{1}{24} g_{P}^{2}, \quad \tilde{c}_{3}^{r}=\tilde{c}_{3}-\frac{1}{6} \tilde{g}_{P}^{2}-\frac{1}{12} g_{P}^{2}$.

We conclude that the terms proportional to $m_{Q}^{2} \bar{I}_{Q}$ or $\bar{I}_{Q}^{(2)}$ in (14) and (16) may be dropped if we use the renormalized low-energy parameters $c_{n}^{r}$ and $\tilde{c}_{n}^{r}$ in the tadpole contributions (15), (17) but also in (24). Note, however, that by doing so some higher-order terms proportional to

$$
\left(1-\frac{M_{R}^{2}}{M_{H}^{2}}\right)^{n} m_{Q}^{2} \bar{I}_{Q} \rightarrow 0,
$$

with $n \geq 1$, are neglected in $\Pi_{H \in\left[1^{-}\right]}$. We argue that the latter terms would cause a renormalization scale dependence that cannot be absorbed into the available counterterms at the considered accuracy level. To avoid a model dependence, such terms should be dropped.

We are left with the terms proportional to $\left(M_{R}^{2}-M_{H}^{2}\right) \bar{I}_{Q}$. If the charm meson masses are decomposed into their chiral moments, the leading renormalization scale dependence of such terms can be absorbed into the $Q^{2}$ counterterms $c_{0,1}$ and $\tilde{c}_{0,1}$. Similarly, the components of order $Q^{4}$ can be matched with counterterms $d_{n}$ and $\tilde{d}_{n}$. Most troublesome, however, are the subleading contributions proportional to $m_{Q}^{5} \log \mu$ in such a strict chiral expansion of the vector $D$-meson masses. There is no counterterm available to remove such a scale dependence. In fact, only within a two-loop computation, this issue is resolved in a conventional approach. Instead, we keep the charm meson masses unexpanded in the terms $\left(M_{R}^{2}-M_{H}^{2}\right) \bar{I}_{Q}$ and follow the strategy proposed in Ref. [35]. For those terms, we provide the decomposition

$$
\left(M_{R}^{2}-M_{H}^{2}\right) \bar{I}_{Q}=\left(M_{R}^{2}-M_{H}^{2}\right) \underbrace{\frac{m_{Q}^{2}}{(4 \pi)^{2}} \log \frac{m_{Q}^{2}}{M_{R}^{2}}}_{=\left.\bar{I}_{Q}\right|_{\mu=M_{R}}}+\frac{M_{R}^{2}-M_{H}^{2}}{M_{R}^{2}} m_{Q}^{2} \underbrace{\bar{I}_{R}}_{\rightarrow 0},
$$

where the second term depending on the heavy-meson tadpole $\bar{I}_{R}$ can be systematically dropped without harming the chiral Ward identities. We end up with the renormalized bubble-loop expressions

$$
\begin{aligned}
\bar{\Pi}_{H \in\left[0^{-}\right]}^{\text {bubble }}= & \sum_{Q \in[8]} \sum_{R \in\left[1^{-}\right]}\left(\frac{G_{Q R}^{(H)}}{2 f}\right)^{2}\left\{\alpha_{Q R}^{H}-M_{H}^{2} p_{Q R}^{2} \bar{I}_{Q R}+\frac{1}{4}\left(M_{R}^{2}-M_{H}^{2}\right) \frac{m_{Q}^{2}}{(4 \pi)^{2}} \log \frac{m_{Q}^{2}}{M_{R}^{2}}\right\} \\
\bar{\Pi}_{H \in\left[1^{-}\right]}^{\text {bubble }}= & \sum_{Q \in[8]} \sum_{R \in\left[0^{-}\right]}\left(\frac{G_{Q R}^{(H)}}{2 f}\right)^{2}\left\{\frac{1}{3} \alpha_{Q R}^{H}-\frac{1}{3} M_{H}^{2} p_{Q R}^{2} \bar{I}_{Q R}+\frac{1}{12}\left(M_{R}^{2}-M_{H}^{2}\right) \frac{m_{Q}^{2}}{(4 \pi)^{2}} \log \frac{m_{Q}^{2}}{M_{R}^{2}}\right\} \\
& +\sum_{Q \in[8]} \sum_{R \in\left[1^{-}\right]}\left(\frac{G_{Q R}^{(H)}}{2 f}\right)^{2}\left\{-\frac{\left(M_{H}^{2}+M_{R}^{2}\right)^{2}}{6 M_{R}^{2}} p_{Q R}^{2} \bar{I}_{Q R}+\frac{\left(M_{H}^{2}+M_{R}^{2}\right)^{2}}{24 M_{H}^{2} M_{R}^{2}}\left(M_{R}^{2}-M_{H}^{2}\right) \frac{m_{Q}^{2}}{(4 \pi)^{2}} \log \frac{m_{Q}^{2}}{M_{R}^{2}}\right\},
\end{aligned}
$$

which will be the basis for our following studies. Note yet the additional subtraction terms $\alpha_{Q R}^{H}$ in (22). Such terms were suggested in Ref. [35] for the analogous case of a baryon self-energy computation. To arrive at consistent results for $m_{Q} \ll \Delta$, the terms $\alpha_{Q R}^{H}$ are instrumental,

$$
\begin{aligned}
& \alpha_{Q R}^{H \in\left[0^{-}\right]}=\frac{\alpha_{1} \Delta^{2}}{32 \pi^{2}}\left\{\left(M_{H}^{2}-M^{2}\right)\left(\frac{\Delta \partial}{\partial M}-\frac{\Delta \partial}{\partial \Delta}-\frac{M+\Delta}{M}\right)+\left(M_{R}^{2}-(M+\Delta)^{2}\right) \frac{M}{M+\Delta}\left(\frac{\Delta \partial}{\partial \Delta}+1\right)\right\} \gamma_{1}+\frac{\Delta M m_{Q}^{2}}{16 \pi^{2}} \alpha_{1} \gamma_{2}, \\
& \alpha_{Q R}^{H \in\left[1^{-}\right]}=\frac{\tilde{\alpha}_{1} \Delta^{2}}{32 \pi^{2}}\left\{\left(M_{H}^{2}-(M+\Delta)^{2}\right) \frac{M}{M+\Delta}\left(\frac{\Delta \partial}{\partial \Delta}+1\right)+\left(M_{R}^{2}-M^{2}\right)\left(\frac{\Delta \partial}{\partial M}-\frac{\Delta \partial}{\partial \Delta}-\frac{M+\Delta}{M}\right)\right\} \tilde{\gamma}_{1}+\frac{\Delta M m_{Q}^{2}}{16 \pi^{2}} \tilde{\alpha}_{1} \tilde{\gamma}_{2},
\end{aligned}
$$


where the functions $\alpha_{i}, \tilde{\alpha}_{i}$ and $\gamma_{i}, \tilde{\gamma}_{i}$ depend on the ratio $\Delta / M$ only. They are listed in Appendices A and B. While the rational functions $\alpha_{i}$ and $\tilde{\alpha}_{i}$ all approach the numerical value 1 in the limit $\Delta \rightarrow 0$, the functions $\gamma_{i}$ and $\tilde{\gamma}_{i}$ show a logarithmic divergence in that limit. We summarize the convenient implications of our subtraction scheme:

(i) The chiral limit values of the $D$-meson masses are not renormalized.

(ii) The low-energy parameters $c_{0,1}$ and $\tilde{c}_{0,1}$ are not renormalized.

(iii) The wave-function factor of the $D$ mesons is not renormalized in the chiral limit.

We close this section with a brief discussion on the role of the renormalization scale $\mu$. Given our scheme, a scale dependence arises from the tadpole terms only. Such terms need to be considered in combination with the tree-level contribution $\Pi_{H}^{(4-\chi)}$. This leads to the condition

$$
\begin{aligned}
\mu^{2} \frac{d}{d \mu^{2}} d_{i}= & -\frac{1}{4} \frac{\Gamma_{d_{i}}}{(4 \pi f)^{2}}, \\
\Gamma_{d_{1}}= & \frac{1}{6}\left(4 c_{1}+12 c_{3}+3 c_{5}\right), \\
\Gamma_{d_{2}}= & \frac{1}{9}\left(44 c_{1}-52 c_{3}-13 c_{5}\right), \\
\Gamma_{d_{3}}= & \frac{1}{18}\left(240 c_{0}-84 c_{1}+240 c_{2}+68 c_{3}\right. \\
& \left.+60 c_{4}+17 c_{5}\right), \\
\Gamma_{d_{4}}= & \frac{1}{27}\left(264 c_{0}-132 c_{1}+264 c_{2}+140 c_{3}\right. \\
& \left.+66 c_{4}+35 c_{5}\right),
\end{aligned}
$$

where identical results hold for the $\tilde{c}_{i}$ and $\tilde{d}_{i}$ coupling constants. However, it is evident that scale-invariant results follow with (24) only if the meson masses in the tadpole contributions are approximated by the leading-order GellMann-Oakes-Renner relations with $m_{\pi}^{2}=2 B_{0} m$ and $m_{K}^{2}=B_{0}\left(m+m_{s}\right)$, for instance. This is unfortunate since we wish to use physical masses inside all loop contributions. Recalling our previous work [35], there may be an efficient remedy of this issue. Indeed, the counterterm contributions can be rewritten in terms of physical masses such that scale invariance follows without insisting on the Gell-Mann-Oakes-Renner relations for the meson masses. Such a rewrite is most economically achieved in terms of suitable linear combinations of the low-energy constants

$$
\begin{aligned}
& d_{1}^{c}=-\frac{1}{23}\left(26 d_{1}+9 d_{2}\right), \\
& d_{3}^{c}=\frac{1}{345}\left(43 d_{1}+60 d_{2}+69 d_{3}\right), \\
& d_{2}^{c}=\frac{1}{276}\left(-132 d_{1}+18 d_{2}\right), \\
& d_{4}^{c}=\frac{1}{45}\left(-11 d_{1}+15 d_{2}-33 d_{3}+45 d_{4}\right) .
\end{aligned}
$$

TABLE III. A rewrite of $\Pi_{H}^{(4-\chi)}$ in (9).

\begin{tabular}{lcc}
\hline \hline$\Pi_{H}^{(4-\chi)}$ & $H=D$ & $H=D_{s}$ \\
\hline$m_{\pi}^{4}$ & $-9 d_{2}^{c}+18 d_{3}^{c}$ & $-18 d_{2}^{c}+18 d_{3}^{c}$ \\
$m_{K}^{4}$ & $-18 d_{2}^{c}+24 d_{3}^{c}$ & $-12 d_{2}^{c}+24 d_{3}^{c}$ \\
$m_{\eta}^{4}$ & $-5 d_{2}^{c}+6 d_{3}^{c}$ & $-2 d_{2}^{c}+6 d_{3}^{c}$ \\
$B_{0} m m_{\pi}^{2}$ & $9 d_{1}^{c}$ & $18 d_{1}^{c}$ \\
$B_{0}\left(m+m_{s}\right) m_{K}^{2}$ & $9 d_{1}^{c}$ & $6 d_{1}^{c}$ \\
$B_{0} m m_{\eta}^{2}$ & $d_{1}^{c}$ & $2 d_{1}^{c}$ \\
$B_{0} m_{s} m_{\eta}^{2}$ & $4 d_{1}^{c}$ & 0 \\
$B_{0}^{2}\left(2 m+m_{s}\right)^{2}$ & $4 d_{4}^{c}$ & $4 d_{4}^{c}$ \\
\hline \hline
\end{tabular}

With Table III, our rewrite is specified in detail. We assure that, replacing the meson masses in the table by their leading-order expressions, the original expressions as given in (9) are recovered identically. We note a particularity: at leading order, the effects of $c_{0}$ in $G_{H Q}^{(\chi)}$ cannot be discriminated from $c_{2}$ in $G_{H Q}^{(S)}$. Scale invariance requires considering the particular combinations $c_{2}+c_{0}$ in $G_{H Q}^{(S)}$ and in turn use $c_{0}=0$ in $G_{H Q}^{(\chi)}$.

\section{SELF-CONSISTENT SUMMATION APPROACH}

The renormalized loop functions depend on the physical masses of the $D$ mesons. In a conventional chiral expansion scheme, the meson masses inside the loop would be expanded to a given order so that a self-consistency issue does not arise. This is fine as long as the expansion is rapidly converging. For a slowly converging system, such a summation scheme is of advantage even though this may bring in some model dependence [32-35].

Let us be specific on how the summation scheme is set up in detail. There is a subtle point emphasized recently in Ref. [35] that needs some discussion. The coupling constant $g_{P}$ was determined in Ref. [6] from the pion-decay width of the $D^{*}$ meson using a tree-level decay amplitude. Alternatively, the decay width can be extracted from the $D^{*}$-meson propagator in the presence of the one-loop polarization $\Pi_{D^{*}}^{\text {buble }}$. The latter has imaginary contributions proportional to the same coupling constant $g_{P}^{2}$ that reflect the considered decay process. In the absence of wavefunction renormalization effects, one would identify a Breit-Wigner width by

$$
M_{D^{*}} \Gamma_{D^{*} \rightarrow D \pi}=-\Im \Pi_{D^{*}}^{\text {bubble }},
$$

where the loop function is evaluated at the $D^{*}$ meson mass $M_{D^{*}}$. Both determinations would provide identical results. However, in the presence of a wave-function renormalization effect from the loop function

$$
Z_{H}-1=\frac{\partial}{\partial M_{H}^{2}} \bar{\Pi}_{H},
$$


this would no longer be the case. Following Ref. [35], we will therefore use the form of the Dyson equation

$M_{H}^{2}-\bar{\Pi}_{H}^{(0)}-\bar{\Pi}_{H}^{(2)}-\bar{\Pi}_{H}^{(4-\chi)}-\bar{\Pi}_{H}^{\text {tadpole }}-\bar{\Pi}_{H}^{\text {bubble }} / Z_{H}=0$,

where we take $\bar{\Pi}_{H}^{(0)}=M^{2}$ and $\bar{\Pi}_{H}^{(0)}=(M+\Delta)^{2}$ for the pseudoscalar and vector $D$ mesons, respectively. The second-order terms $\bar{\Pi}_{H}^{(2)}$ are the tree-level contributions (9) proportional to the quark masses as written in terms of the parameters $c_{0}, c_{1}$ and $\tilde{c}_{0}, \tilde{c}_{1}$. The fourth-order terms $\bar{\Pi}_{H}^{(4-\chi)}$ are the tree-level contributions (9) proportional to the product of two quark masses. Here, the parameters $d_{i}$ and $\tilde{d}_{i}$ are probed. We recall that the wave-function renormalization $Z_{H}$ has a quark-mass dependence that cannot be fully moved into the counterterms of the chiral Lagrangian.

We provide a first numerical estimate of the importance of the various terms in (28). We put $\bar{\Pi}_{H}^{(4-\chi)}=\bar{\Pi}_{H}^{\text {tadpole }}=0$ since the associated counterterms are not known reliably. Insisting on the large- $N_{c}$ relations

$$
2 c_{0}=c_{1}, \quad 2 \tilde{c}_{0}=\tilde{c}_{1},
$$

we adjust the four parameters $c_{1}, \tilde{c}_{1}$ and $M, \Delta$ to the four isospin-averaged pseudoscalar and vector $D$-meson masses [43]. The results of this procedure are collected in the second-to-last column of Table IV. In the third column, we show the size of the loop contribution $\bar{\Pi}_{H}^{\text {bubble }}$ and the wave-function renormalization factor $Z_{H}$. From those numbers we conclude that the loop terms are as important as the contributions of the $Q^{2}$ counterterms (shown in the second column). Note also the significant size of the wave-function factor for the strange $D$ mesons. It is instructive to compare the values of the four parameters $c_{1}, \tilde{c}_{1}$ and $M, \Delta$ with their corresponding values that follow in a scenario in which all loop effects are neglected. Such values are shown in the last column of Table IV. A reasonable spread of the parameters as compared to the initial scenario is observed.

While with (28) we arrive at a renormalization scale-invariant and self-consistent approach for a chiral extrapolation of the $D$-meson masses that considers all counterterms relevant at next-to-next-to-next-to-leadingorder $\left(\mathrm{N}^{3} \mathrm{LO}\right)$, there is an important issue remaining. Is it possible to decompose the renormalized loop function $\bar{\Pi}_{H}^{\text {bubble }}$ into its chiral moments and therewith shed more light on the convergence properties of such a chiral expansion. It is known that a conventional chiral expansion has not too convincing convergence properties at physical values of the strange quark mass. Does a resummed scheme that is formulated in terms of physical meson masses show an improved convergence pattern?

\section{POWER-COUNTING DECOMPOSITION OF THE LOOP FUNCTION}

At sufficiently small quark masses, a linear dependence of the $D$-meson masses is expected as recalled in (9). The associated slope parameters $c_{0}, c_{1}$ and $\tilde{c}_{0}, \tilde{c}_{1}$ are scale independent. This is an effect of chiral order $Q^{2}$. With increasing quark masses, additional terms in the chiral expansion turn relevant. While there is no controversy on how to count the $Q^{4}$ contributions $\bar{\Pi}_{H}^{(4-\chi)}$ and $\bar{\Pi}_{H}^{\text {tadpole }}$, it is less obvious how to further decompose the loop contribution $\bar{\Pi}_{H}^{\text {bubble }}$ into its power-counting moments. The loop functions depend on the physical masses $m_{Q}, M_{H}$, and $M_{R}$. In any power-counting ansatz based on chiral dynamics, we would assign

$$
\frac{m_{Q}}{M_{R}} \sim Q \sim \frac{m_{Q}}{M_{H}}
$$

for the ratios of the Goldstone boson masses over the $D$ meson masses. The mass differences of either pseudoscalar or vector mesons

$$
\frac{M_{H}-M_{R}}{m_{Q}} \sim Q, \quad \frac{M_{R}-M_{H}}{M_{H}} \sim Q^{2} \quad \text { for } H \| R,
$$

can also be counted without controversy. In (31), we use a notation $H \| R$ requesting $H, R \in\left[0^{-}\right]$or $H, R \in\left[1^{-}\right]$. Less obvious is how to treat the mass differences of a pseudoscalar and a vector $D$ meson.

There are different schemes possible. Technically, most straightforward is the extreme assumption

$$
\frac{M_{R}-M_{H}}{m_{Q}} \sim Q, \quad \frac{M_{R}-M_{H}}{M_{H}} \sim Q^{2} \quad \text { for } H \perp R,
$$

TABLE IV. The loop functions (22) are evaluated with the coupling constants $g_{P}=\tilde{g}_{P} \simeq 0.57$ and the physical isospin-averaged meson masses. In addition, the large- $N_{c}$ relations (29) are assumed.

\begin{tabular}{lrrcccc}
\hline \hline$H$ & $\bar{\Pi}_{H}^{(2)} /\left(2 M_{H}\right)$ & $\bar{\Pi}_{H}^{\text {bubble }} /\left(2 M_{H}\right)$ & $Z_{H}$ & & With bubble & Tree level \\
\hline$D$ & $4.7 \mathrm{MeV}$ & $-50.2 \mathrm{MeV}$ & 1.108 & $M$ & $1907.4 \mathrm{MeV}$ & $1862.7 \mathrm{MeV}$ \\
$D_{s}$ & $106.2 \mathrm{MeV}$ & $-65.5 \mathrm{MeV}$ & 1.418 & $\Delta$ & $191.7 \mathrm{MeV}$ & $141.3 \mathrm{MeV}$ \\
$D^{*}$ & $5.0 \mathrm{MeV}$ & $-113.4 \mathrm{MeV}$ & 1.163 & $c_{1}$ & 0.440 & 0.426 \\
$D_{s}^{*}$ & $114.1 \mathrm{MeV}$ & $-166.1 \mathrm{MeV}$ & 1.643 & $\tilde{c}_{1}$ & 0.508 & 0.469 \\
\hline \hline
\end{tabular}


which can be motivated in the limit of a large charm-quark mass at which $\Delta \rightarrow 0$ and therewith $\Delta \ll m_{\pi}$. In (32), we use a notation $H \perp R$, implying that either $H \in\left[0^{-}\right]$and $R \in\left[1^{-}\right]$or $H \in\left[1^{-}\right]$and $R \in\left[0^{-}\right]$. While the counting ansatz (32) is expected to be faithful for $m_{Q}=m_{K}$, it is not so useful for $m_{Q}=m_{\pi}$. However, since the loop corrections are typically dominated by contributions involving the kaon and eta meson masses, such an assumption should have some qualitative merits nevertheless. The leading-order terms are readily worked out with

$$
\begin{aligned}
\bar{\Pi}_{H \in\left[0^{-}\right]}^{\text {bubble }}= & \sum_{Q \in[8]} \sum_{R \in\left[1^{-}\right]}\left(\frac{G_{Q R}^{(H)}}{2 f}\right)^{2}\left\{\alpha_{Q R}^{H}+X_{Q R}^{(H)}+\frac{\gamma_{R}^{H}}{16 \pi^{2}} M_{H}^{2} m_{Q}^{2}\left[1-\left(\frac{m_{Q}}{2 M_{H}}-\frac{M_{R}-M_{H}}{m_{Q}}\right)^{2}\right]\right\}+\mathcal{O}\left(Q^{6}\right), \\
\bar{\Pi}_{H \in\left[1^{-}\right]}^{\text {bubble }}= & \frac{2}{3} \sum_{Q \in[8]} \sum_{R \in\left[1^{-}\right]}\left(\frac{G_{Q R}^{(H)}}{2 f}\right)^{2} X_{Q R}^{(H)} \\
& +\frac{1}{3} \sum_{Q \in[8]} \sum_{R \in\left[0^{-}\right]}\left(\frac{G_{Q R}^{(H)}}{2 f}\right)^{2}\left\{\alpha_{Q R}^{H}+X_{Q R}^{(H)}+\frac{\gamma_{R}^{H}}{16 \pi^{2}} M_{H}^{2} m_{Q}^{2}\left[1-\left(\frac{m_{Q}}{2 M_{H}}-\frac{M_{R}-M_{H}}{m_{Q}}\right)^{2}\right]\right\}+\mathcal{O}\left(Q^{6}\right), \\
X_{Q R}^{(H)}= & M_{H}^{2} \frac{m_{Q}^{2}}{16 \pi^{2}}\left(\frac{m_{Q}^{2}}{M_{H}^{2}}+2 \frac{M_{R}-M_{H}}{M_{H}}\right)-M_{H}^{2} \frac{m_{Q}^{2}}{32 \pi^{2}}\left(\frac{m_{Q}^{2}}{M_{H}^{2}}-3 \frac{M_{R}-M_{H}}{M_{H}}\right) \log \frac{m_{Q}^{2}}{M_{R}^{2}} \\
& +M_{H} \frac{m_{Q}^{3}}{16 \pi^{2}}\left[-\pi+\frac{3 \pi}{2}\left(\frac{m_{Q}}{2 M_{H}}-\frac{M_{R}-M_{H}}{m_{Q}}\right)^{2}\right],
\end{aligned}
$$

accurate to order $Q^{5}$. The coefficients $\alpha_{Q R}^{H}$ and $\gamma_{R}^{H}$ were given already in (23) and (11). In Table V, we decompose the loop function into third-, fourth-, and fifth-order numerical values. The results are compared with the exact numbers already shown in Table IV. While we observe a qualitative reproduction of the full loop function, owing to contributions from intermediate pion states, there is no convergence observed, as expected. By construction, the counting rule (32) fails in the chiral regime where all quark masses, in particular, the strange-quark mass, approach zero. This is illustrated by Fig. 1, in which we plot the loop function $\bar{\Pi}_{H}$ in the flavor limit with $m_{\pi}=m_{K}=m_{\eta}$. Here, the $D$-meson masses $M_{D}=M_{D_{s}}$ and $M_{D^{*}}=M_{D_{s}^{*}}$ are obtained as the solution of the set of Dyson equations (28) in which the full loop expression (22) is assumed. The parameter set of Table IV, which is based on the scenario $\bar{\Pi}_{H}^{(4-\chi)}=\bar{\Pi}_{H}^{\text {tadpole }}=0$, is applied. While for large pion masses the hierarchy of dashed and dotted lines systematically approaches the solid line, this is not the case for pion masses smaller than $m_{\pi} \leq \Delta \sim 200 \mathrm{MeV}$.
How can one improve on the counting rule (32)? Before presenting a universal approach, we consider yet two further interim power-counting scenarios. First, we work out the extreme chiral region where all Goldstone boson masses are significantly smaller than $\Delta \sim 200 \mathrm{MeV}$. In this case, the counting rules

$$
\frac{m_{Q}}{\Delta} \sim Q, \quad \frac{\Delta}{M} \sim Q^{0}
$$

are used. Since the extreme chiral region is not realized in nature, such an assumption is not expected to provide any significant results for quantities measurable in experimental laboratories.

Since at some stage lattice QCD simulations may be feasible at such low strange-quark masses, we provide the corresponding expressions for the loop function nevertheless. Here, we decompose all meson masses into their chiral moments in application of a strict chiral expansion. At third order,

TABLE V. The loop functions (22) are evaluated with the coupling constants $g_{P}=\tilde{g}_{P} \simeq 0.57$ and the physical isospin-averaged meson masses. A decomposition according to (30), (31), and (32) is performed. All terms are in units of MeV.

\begin{tabular}{lcccc}
\hline \hline$H$ & $\bar{\Pi}_{H}^{\text {bubble }} /\left(2 M_{H}\right)$ & $\bar{\Pi}_{H}^{\text {bubble-3 }} /\left(2 M_{H}\right)$ & $\bar{\Pi}_{H}^{\text {bubble-4 }} /\left(2 M_{H}\right)$ & $\bar{\Pi}_{H}^{\text {bubble-5 }} /\left(2 M_{H}\right)$ \\
\hline$D$ & -50.2 & -38.7 & -29.4 & 22.8 \\
$D_{s}$ & -65.5 & -93.2 & 27.3 & 2.4 \\
$D^{*}$ & -113.4 & -135.1 & 19.0 & 6.3 \\
$D_{s}^{*}$ & -166.1 & -308.3 & 99.8 & 61.8 \\
\hline \hline
\end{tabular}



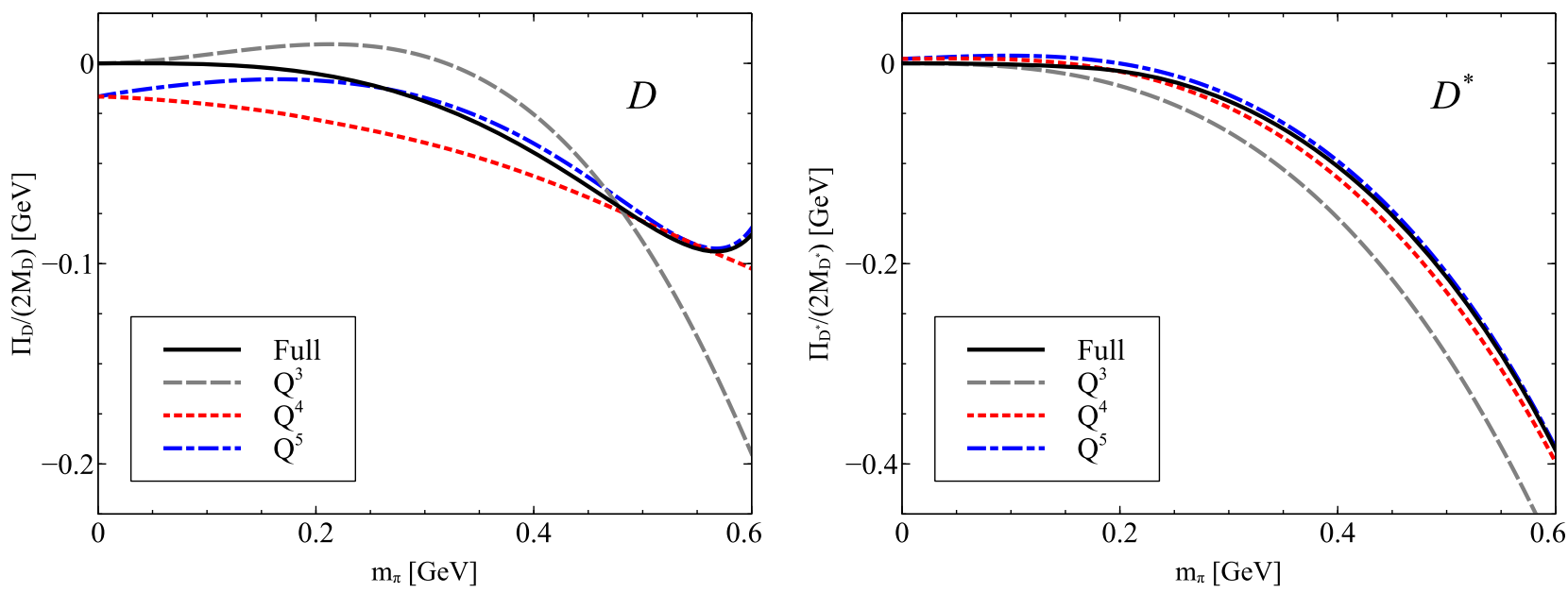

FIG. 1. $D$ - and $D^{*}$-meson masses in the flavor limit as a function of the pion mass. The power-counting decomposed loop functions of (33) are used with the parameter set of Table IV.

$\Pi_{H \in\left[0^{-}\right]}^{\text {bubble-3 }}=0$

$\Pi_{H \in\left[1^{-}\right]}^{\text {bubble-3 }}=-\frac{2}{3} \pi \sum_{Q \in[8]} \sum_{R \in\left[1^{-}\right]}\left(\frac{G_{Q R}^{H}}{8 \pi f}\right)^{2} m_{Q}^{3}(M+\Delta)$,

the vector $D$ mesons pick up a contribution only. At fourth order, the expressions turn more complicated. We do not expand in powers of $\Delta / M$ because there are terms present proportional to $\log \Delta / M$ and also because we do not want to pollute the strict chiral expansion by a further scale assumption. The algebra required is somewhat involved, and we organize it by a series of suitable dimensionless coefficients $\alpha_{n}, \gamma_{n}$ and $\tilde{\alpha}_{n}, \tilde{\gamma}_{n}$ that depend on the ratio $\Delta / M$ only. While the coefficients $\gamma_{n}, \tilde{\gamma}_{n}$ characterize the chiral expansion of the scalar bubble functions, the $\alpha_{n}, \tilde{\alpha}_{n}$ result from a chiral expansion of the coefficients in front of the scalar loop functions. Altogether, we derive the compact expressions

$$
\begin{aligned}
\bar{\Pi}_{H \in\left[0^{-}\right]}^{\text {bubble-4 }}= & \sum_{Q \in[8]} \sum_{R \in\left[1^{-}\right]}\left(\frac{G_{Q R}^{H}}{8 \pi f}\right)^{2}\left\{\gamma_{d}^{(1)} m_{Q}^{2} \Pi_{R}^{(2)}+\gamma_{d}^{(2)} m_{Q}^{2} \Pi_{H}^{(2)}+\gamma_{d}^{(3)} \Pi_{R}^{(2)} \Pi_{R}^{(2)}+\gamma_{d}^{(4)} \Pi_{H}^{(2)} \Pi_{H}^{(2)}\right. \\
& \left.+\gamma_{d}^{(5)} \Pi_{R}^{(2)} \Pi_{H}^{(2)}+\frac{M}{\Delta} m_{Q}^{4}\left[\left(\alpha_{2} \gamma_{2}-\alpha_{1} \gamma_{4}\right)+\left(\alpha_{2} \gamma_{3}-\alpha_{1} \gamma_{5}\right) \log \frac{m_{Q}^{2}}{(M+\Delta)^{2}}\right]\right\}, \\
\bar{\Pi}_{H \in\left[1^{-}\right]}^{\text {bubble-4 }}= & \sum_{Q \in[8]} \sum_{R \in\left[0^{-}\right]}\left(\frac{G_{Q R}^{H}}{8 \pi f}\right)^{2}\left\{\tilde{\gamma}_{d}^{(1)} m_{Q}^{2} \Pi_{R}^{(2)}+\tilde{\gamma}_{d}^{(2)} m_{Q}^{2} \Pi_{H}^{(2)}+\tilde{\gamma}_{d}^{(3)} \Pi_{R}^{(2)} \Pi_{R}^{(2)}+\tilde{\gamma}_{d}^{(4)} \Pi_{H}^{(2)} \Pi_{H}^{(2)}\right. \\
& \left.+\tilde{\gamma}_{d}^{(5)} \Pi_{R}^{(2)} \Pi_{H}^{(2)}+\frac{M}{3 \Delta} m_{Q}^{4}\left[\left(\tilde{\alpha}_{2} \tilde{\gamma}_{2}-\tilde{\alpha}_{1} \tilde{\gamma}_{4}\right)+\left(\tilde{\alpha}_{2} \tilde{\gamma}_{3}-\tilde{\alpha}_{1} \tilde{\gamma}_{5}\right) \log \frac{m_{Q}^{2}}{M^{2}}\right]\right\} \\
& +\sum_{Q \in[8]} \sum_{R \in\left[1^{-}\right]}\left(\frac{G_{Q R}^{H}}{8 \pi f}\right)^{2} \frac{2}{3}\left\{m_{Q}^{2}\left(m_{Q}^{2}-\Pi_{H}^{(2)}+\Pi_{R}^{(2)}\right)-\frac{1}{4}\left(2 m_{Q}^{2}+3 \Pi_{H}^{(2)}-3 \Pi_{R}^{(2)}\right) m_{Q}^{2} \log \frac{m_{Q}^{2}}{(M+\Delta)^{2}}\right\} .
\end{aligned}
$$

The dimensionless coefficients $\gamma_{d}^{(n)}$ and $\tilde{\gamma}_{d}^{(k)}$ are expressed in terms of the basic coefficients $\alpha_{n}, \gamma_{n}$ and $\tilde{\alpha}_{n}, \tilde{\gamma}_{n}$ in Appendices A and B. Again, they depend on the ratio $\Delta / M$ only. We note that the rational functions $\alpha_{n}$ and $\tilde{\alpha}_{n}$ approach 1 in the limit $\Delta / M \rightarrow 0$. In contrast, the $\gamma_{n}$ and $\tilde{\gamma}_{n}$ have contributions proportional to $\log \Delta / M$ and do not approach 1 in the heavy quark-mass limit. All terms in (36) that are proportional to $\gamma_{d}^{(n)}$ or $\tilde{\gamma}_{d}^{(n)}$ can be viewed as a renormalization of the low-energy parameters $d_{n}$ and $\tilde{d}_{n}$. This is illustrated in Appendices A and B, in which explicit expressions are provided. We note that the fifth-order terms can also be readily constructed. For the vector $D$ mesons, we derive 


$$
\begin{aligned}
\bar{\Pi}_{H \in\left[1^{-}\right]}^{\text {bubble-5 }}= & \sum_{Q \in[8]} \sum_{R \in\left[1^{-}\right]}\left(\frac{G_{Q R}^{(H)}}{8 \pi f}\right)^{2} \frac{\pi m_{Q}}{12(M+\Delta)} \\
& \times\left\{3 m_{Q}^{4}+m_{Q}^{2}\left(2 \Pi_{H}^{(2)}-6 \Pi_{R}^{(2)}\right)\right. \\
& \left.+3\left(\Pi_{H}^{(2)}-\Pi_{R}^{(2)}\right)^{2}\right\}+\cdots,
\end{aligned}
$$

where the dots stand for additional terms extracted from (36) with the replacement $\Pi_{H}^{(2)} \rightarrow \Pi_{H}^{(3)}$. For the pseudoscalar $D$ mesons, the corresponding expressions follow from (36) with the replacement $\Pi_{H}^{(2)} \rightarrow \Pi_{H}^{(3)}$ only.

We plot the loop function $\bar{\Pi}_{H}$ in the flavor limit with $m_{\pi}=m_{K}=m_{\eta}$ and $M_{D}=M_{D_{s}}=M$ and $M_{D^{*}}=M_{D_{s}^{*}}=$ $M+\Delta$. Here, we use our first estimate for the low-energy parameters $c_{0,1}$ and $\tilde{c}_{0,1}$ as displayed in the next-to-last column of Table IV. From Fig. 2, we conclude that for pion masses smaller than $\Delta$ (the value of which is indicated by the vertical line in light gray) the successive orders (dashed, dotted, and dash-dotted lines) approach the exact solid line convincingly. Unlike the consequences of the powercounting ansatz (32) as illustrated in the previous Fig. 1, this is clearly not the case for (34) in the large pion-mass domain with $m_{\pi}>\Delta$.

Neither the extreme counting assumptions (32) nor (34) generates an expansion scheme that converges for physical up, down, and strange quark masses. A step forward may be provided by the conventional ansatz

$\Delta \sim m_{Q} \sim Q, \quad \Delta_{Q}=\sqrt{\Delta^{2}-m_{Q}^{2}} \sim Q, \quad \frac{\Delta}{M} \sim Q$,

suggested originally by Banerjee and Milana $[44,45]$ for the chiral expansion of baryon masses. Even though the authors demonstrated in a recent work [35] that such an expansion is not suitable to arrive at a meaningful expansion for the baryon octet and decuplet masses at physical values of the up, down, and strange quark masses, it deserves a closer study of whether it may prove significant for a chiral expansion of the $D$-meson masses. The counting rules (38) lead to somewhat more complicated expressions. Again, we derive the third-, fourth-, and fifthorder terms. We find

$$
\begin{aligned}
\bar{\Pi}_{H \in\left[0^{-}\right]}^{\text {bubble-3 }}= & \sum_{Q \in[8]} \sum_{R \in\left[1^{-}\right]}\left(\frac{G_{Q R}^{(H)}}{8 \pi f}\right)^{2}\left\{-\Delta_{Q}^{3} M\left(\log \left(\Delta+\Delta_{Q}\right)-\log \left(\Delta-\Delta_{Q}\right)\right)-\Delta M\left(\Delta_{Q}^{2}-\frac{1}{2} m_{Q}^{2}\right) \log \frac{m_{Q}^{2}}{4 \Delta^{2}}-\frac{1}{2} \Delta M m_{Q}^{2}\right\}, \\
\bar{\Pi}_{H \in\left[1^{-}\right]}^{\text {bubble-3 }}= & \sum_{Q \in[8]} \sum_{R \in\left[1^{-}\right]}\left(\frac{G_{Q R}^{(H)}}{8 \pi f}\right)^{2} \frac{2}{3}\left\{-\pi M m_{Q}^{3}\right\}+\sum_{Q \in[8]} \sum_{R \in\left[0^{-}\right]}\left(\frac{G_{Q R}^{(H)}}{8 \pi f}\right)^{2} \frac{1}{3}\left\{\Delta_{Q}^{3} M\left(\log \left(-\Delta-\Delta_{Q}\right)-\log \left(-\Delta+\Delta_{Q}\right)\right)\right. \\
& \left.+\Delta M\left(\Delta_{Q}^{2}-\frac{1}{2} m_{Q}^{2}\right) \log \frac{m_{Q}^{2}}{4 \Delta^{2}}+\frac{1}{2} \Delta M m_{Q}^{2}\right\},
\end{aligned}
$$

and
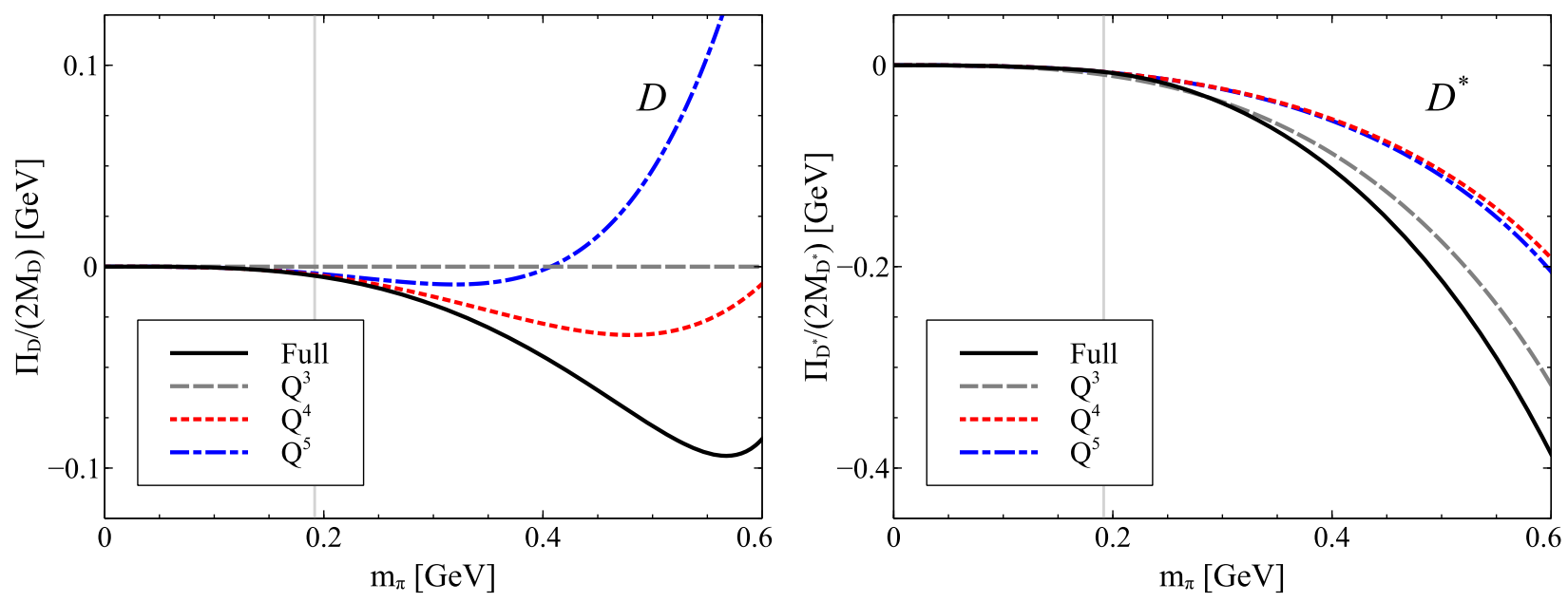

FIG. 2. $D$-meson masses in the flavor limit as a function of the pion mass. The power-counting decomposed loop functions of (35), (36), (37) are used with the parameter set of Table IV. 
TABLE VI. The loop functions (22), (40) are evaluated with the coupling constants $g_{P}=\tilde{g}_{P} \simeq 0.57$ and the physical isospin-averaged meson masses. A decomposition according to (38) is performed. All terms are in units of $\mathrm{MeV}$.

\begin{tabular}{lcccr}
\hline \hline$H$ & $\bar{\Pi}_{H}^{\text {bubble }} /\left(2 M_{H}\right)$ & $\bar{\Pi}_{H}^{\text {bubble-3 }} /\left(2 M_{H}\right)$ & $\bar{\Pi}_{H}^{\text {bubble-4 }} /\left(2 M_{H}\right)$ & $\bar{\Pi}_{H}^{\text {bubble-5 }} /\left(2 M_{H}\right)$ \\
\hline$D$ & -50.2 & -67.7 & 15.0 & -8.9 \\
$D_{s}$ & -65.6 & -152.8 & 27.8 & 26.6 \\
$D^{*}$ & -113.4 & -111.7 & -57.1 & 18.6 \\
$D_{s}^{*}$ & -166.1 & -252.0 & 84.3 & -69.5 \\
\hline \hline
\end{tabular}

$$
\begin{aligned}
\bar{\Pi}_{H \in\left[0^{-}\right]}^{\text {bubble-4 }}= & \sum_{Q \in[8]} \sum_{R \in\left[1^{-}\right]}\left(\frac{G_{Q R}^{(H)}}{8 \pi f}\right)^{2}\left\{\frac{1}{4}\left(-3 \Delta^{2}+4 m_{Q}^{2}-4 \Pi_{H}^{(2)}+4 \Pi_{R}^{(2)}\right) m_{Q}^{2}-\frac{3}{2} \Delta^{2}\left(\Delta_{Q}^{2}-\Pi_{H}^{(2)}+\Pi_{R}^{(2)}-\frac{1}{2} m_{Q}^{2}\right) \log \frac{m_{Q}^{2}}{4 \Delta^{2}}\right. \\
& \left.-\frac{1}{4}\left(2 m_{Q}^{2}+3 \Pi_{H}^{(2)}-3 \Pi_{R}^{(2)}\right) m_{Q}^{2} \log \frac{m_{Q}^{2}}{M^{2}}-\Delta_{Q} M\left(\log \left(\Delta+\Delta_{Q}\right)-\log \left(\Delta-\Delta_{Q}\right)\right) \frac{3 \Delta}{2 M}\left(\Delta_{Q}^{2}-\Pi_{H}^{(2)}+\Pi_{R}^{(2)}\right)\right\} \\
\bar{\Pi}_{H \in\left[1^{-}\right]}^{\text {bubble-4 }}= & \sum_{Q \in[8]} \sum_{R \in\left[1^{-}\right]}\left(\frac{G_{Q R}^{(H)}}{8 \pi f}\right)^{2} \frac{2}{3}\left\{-\pi \Delta m_{Q}^{3}+\left(m_{Q}^{2}-\Pi_{H}^{(2)}+\Pi_{R}^{(2)}\right) m_{Q}^{2}-\frac{1}{4}\left(2 m_{Q}^{2}+3 \Pi_{H}^{(2)}-3 \Pi_{R}^{(2)}\right) m_{Q}^{2} \log \frac{m_{Q}^{2}}{M^{2}}\right\} \\
& +\sum_{Q \in[8]} \sum_{R \in\left[0^{-}\right]}\left(\frac{G_{Q R}^{(H)}}{8 \pi f}\right)^{2} \frac{1}{3}\left\{\frac{1}{4}\left(-\Delta^{2}+4 m_{Q}^{2}-4 \Pi_{H}^{(2)}+4 \Pi_{R}^{(2)}\right) m_{Q}^{2}-\frac{\Delta^{2}}{2}\left(\Delta_{Q}^{2}-3 \Pi_{H}^{(2)}+3 \Pi_{R}^{(2)}-\frac{1}{2} m_{Q}^{2}\right) \log \frac{m_{Q}^{2}}{4 \Delta^{2}}\right. \\
& -\frac{1}{4}\left(2 m_{Q}^{2}+3 \Pi_{H}^{(2)}-3 \Pi_{R}^{(2)}\right) m_{Q}^{2} \log \frac{m_{Q}^{2}}{M^{2}}-\Delta_{Q} M\left(\log \left(-\Delta-\Delta_{Q}\right)-\log \left(-\Delta+\Delta \Delta_{Q}\right) \frac{\Delta}{2 M}\left(\Delta_{Q}^{2}-3 \Pi_{H}^{(2)}+3 \Pi_{R}^{(2)}\right)\right\},
\end{aligned}
$$

with $\Delta_{Q}$ of (38). Since the fifth-order contributions are quite lengthy, they are delegated to Appendices A and B. In Table VI, we decompose the loop function into third-, fourth-, and fifth-order numerical values. The results are compared with the exact numbers already shown in Table IV. The conclusions of that table are unambiguous: the power-counting ansatz (38) is not suitable for a chiral extrapolation of the $D$-meson masses. We note that (38)

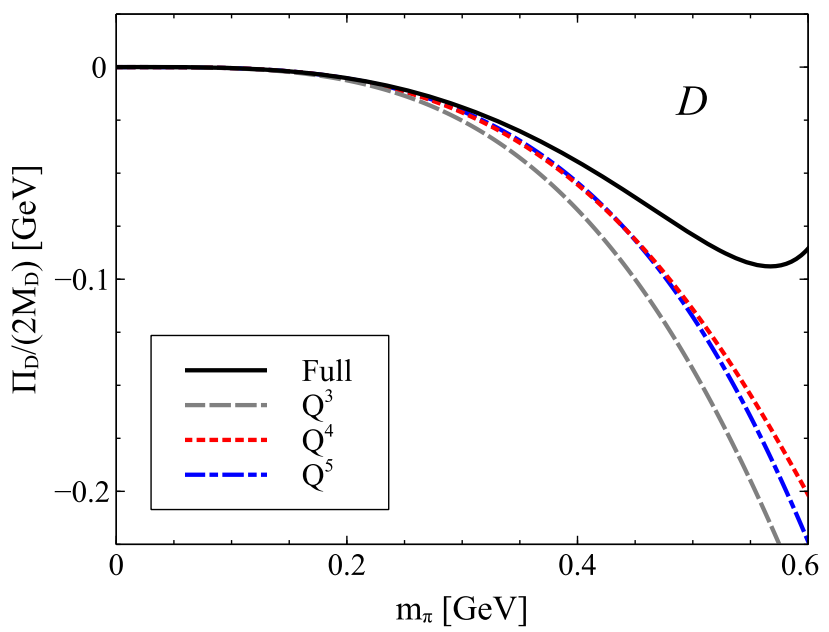

reproduces neither the results of (32) nor those of (34). We further demonstrate our claim by a plot of the loop function $\bar{\Pi}_{H}$ in the flavor limit with $m_{\pi}=m_{K}=m_{\eta}$ as was done in Figs. 1 and 2. Figure 3 demonstrates that for $m_{\pi}>\Delta$ no quantitative reproduction of the solid line is obtained.

We finally present our counting ansatz that is expected to be applicable from small- to medium-size quark masses uniformly. It is an adaptation of the framework developed

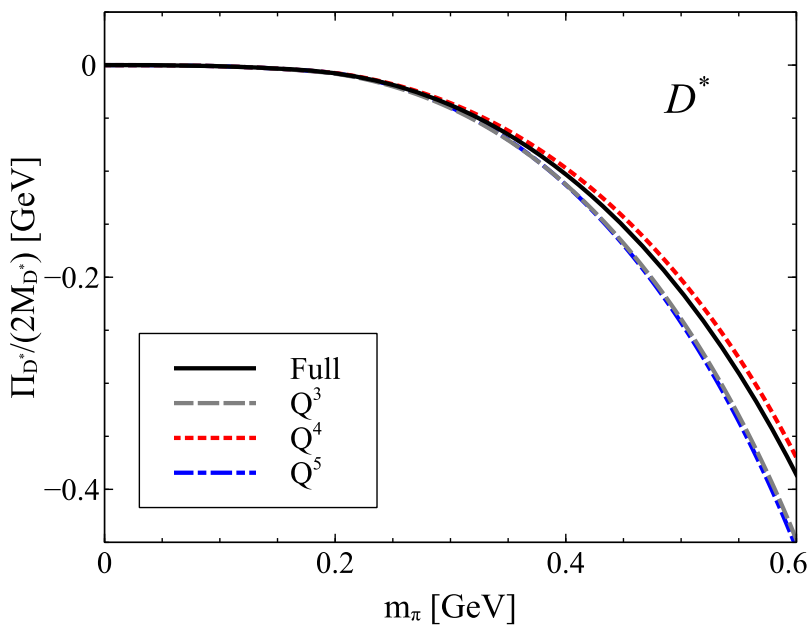

FIG. 3. D-meson masses in the flavor limit as a function of the pion mass. The power-counting decomposed loop functions of (39), (40), (A5), and (B4) are used with the parameter set of Table IV. 
recently for the chiral extrapolation of the baryon octet and decuplet masses [35] and implements the driving idea to formulate the expansion coefficients in terms of physical masses. It is supposed to interpolate the two extreme counting rules (32) and (34). The counting rules are

$$
\begin{aligned}
& \frac{M_{R}-M_{H}}{m_{Q}} \sim Q, \quad \frac{M_{R}-M_{H}}{M_{H}} \sim Q^{2} \quad \text { for } H \| R \\
& \frac{M_{R}-M_{H}}{m_{Q}} \sim Q^{0}, \quad \frac{M_{R}-M_{H} \pm \Delta_{H}}{M_{H}} \sim Q^{2} \quad \text { for } H \perp R \\
& \Delta_{Q}=\sqrt{\left(M_{H}-M_{R}\right)^{2}-m_{Q}^{2}} \sim Q \quad \text { with } \\
& \Delta_{H}=\Delta M_{H} \lim _{m_{u, d, s} \rightarrow 0} \frac{1}{M_{H}},
\end{aligned}
$$

where the sign \pm is chosen such that the last ratio in (41) vanishes in the chiral limit. The implications of (41) are more difficult to work out. The counting rules (41) as they are necessarily imply

$$
Q \sim \frac{\Delta_{H}}{M_{H}}=\left\{\begin{array}{cl}
\frac{\Delta}{M} & \text { for } H \in\left[0^{-}\right] \\
\frac{\Delta}{M+\Delta} & \text { for } H \in\left[1^{-}\right]
\end{array},\right.
$$

which is at odds with the assumption in (34). Therefore, we supplement (41) by the request that the implications of (41) are recovered in the chiral regime. This requires a particular summation of terms proportional to $(\Delta / M)^{n}$ with $n=1,2,3, \ldots$.

There is yet another issue pointed out in Ref. [35]. The chiral expansion of the scalar bubble function is characterized by an alternating feature. We recall from Ref. [35] the approximation hierarchy

$(4 \pi)^{2} \bar{I}_{Q R}=-\left\{1-\frac{1}{8} x^{2}-\frac{1}{128} x^{4}-\frac{1}{1024} x^{6}+\mathcal{O}\left(x^{8}\right)\right\} \pi \sqrt{x^{2}}+\left\{1-\frac{1}{12} x^{2}-\frac{1}{120} x^{4}-\frac{1}{840} x^{6}+\mathcal{O}\left(x^{8}\right)\right\} x^{2}-\frac{1}{2} x^{2} \log x^{2}$,

where we denoted $x=m_{Q} / M_{H}$ and $M_{R}=M_{H}$. As was discussed in Ref. [35], the terms with even and odd powers in $x$ have opposite signs always. This implies a systematic cancellation effect among terms proportional to $x^{n}$ and $x^{1+n}$, where the effect is most striking for $n=1$. Therefore, it is useful to always group such terms together. Even though the need of such a reorganization is not very strong for the $D$-meson systems under consideration, we adapt this strategy in the following. Note that the convergence domain of (43) was proven to be limited by $|x|<2$ only, a surprisingly large convergence circle. Given this scheme, accurate results can be obtained by a few leading-order terms. We construct the third-order contributions from the one-loop diagrams,

$$
\begin{aligned}
\bar{\Pi}_{H \in\left[0^{-}\right]}^{\text {buble- }}= & \sum_{Q \in[8], R \in\left[1^{-}\right]}\left(\frac{G_{Q R}^{(H)}}{4 \pi f}\right)^{2} \frac{\alpha_{1}}{4}\left\{\delta_{7} \Delta M m_{Q}^{2}+\delta_{6} \Delta^{2} M\left(M_{R}-M_{H}-\Delta_{H}\right)+M_{H}\left[\Delta_{Q}^{2}\left(\gamma_{1} \Delta_{H}-\delta_{1}\left(M_{R}-M_{H}\right)\right)\right.\right. \\
& -\frac{2 M+\Delta}{2 M}\left(\left(M_{R}-M_{H}\right)\left(\Delta_{Q}^{2}-\frac{1}{2} m_{Q}^{2}\right) \log \frac{m_{Q}^{2}}{M_{R}^{2}}+\Delta_{Q}^{3}\left[\log \left(M_{R}-M_{H}+\Delta_{Q}\right)-\log \left(M_{R}-M_{H}-\Delta_{Q}\right)\right]\right) \\
& \left.\left.+\frac{m_{Q}^{2}}{\Delta_{H}}\left(-\delta_{2} \Delta_{Q}^{2}+\delta_{3} m_{Q}^{2} \log \frac{m_{Q}^{2}}{M_{R}^{2}}\right)\right]\right\}, \\
\bar{\Pi}_{H \in\left[1^{-}\right]}^{\text {buble-3 }}= & \sum_{Q \in[8], R \in\left[1^{-}\right]}\left(\frac{G_{Q R}^{(H)}}{4 \pi f}\right)^{2} \frac{M_{H}}{6}\left\{\frac{m_{Q}^{2}}{M_{H}}\left(1-\log \frac{m_{Q}}{M_{R}}\right)-\pi m_{Q}\right\}\left(m_{Q}^{2}-\left(M_{H}-M_{R}\right)^{2}\right) \\
& +\sum_{Q \in[8], R \in\left[0^{-}\right]}\left(\frac{G_{Q R}^{(H)}}{4 \pi f}\right)^{2} \frac{\tilde{\alpha}_{1}}{12}\left\{\tilde{\delta}_{7} \Delta m_{Q}^{2}-\tilde{\delta}_{6} \Delta^{2} M\left(M_{R}-M_{H}+\Delta_{H}\right)\right. \\
& +M_{H} \frac{M}{M+\Delta}\left[\Delta_{Q}^{2}\left(\tilde{\gamma}_{1} \Delta_{H}-\tilde{\delta}_{1}\left(M_{H}-M_{R}\right)\right)+\frac{M(2 M+\Delta)}{2(M+\Delta)^{2}}\left(\left(M_{H}-M_{R}\right)\left(\Delta_{Q}^{2}-\frac{1}{2} m_{Q}^{2}\right) \log \frac{m_{Q}^{2}}{M_{R}^{2}}\right.\right. \\
& \left.\left.\left.+\Delta_{Q}^{3}\left[\log \left(M_{R}-M_{H}-\Delta_{Q}\right)-\log \left(M_{R}-M_{H}+\Delta_{Q}\right)\right]\right)+\frac{m_{Q}^{2}}{\Delta_{H}}\left(-\tilde{\delta}_{2} \Delta_{Q}^{2}+\tilde{\delta}_{3} m_{Q}^{2} \log \frac{m_{Q}^{2}}{M_{R}^{2}}\right)\right]\right\},
\end{aligned}
$$

with $\Delta_{Q}$ and $\Delta_{H}$ as introduced in (41). The dimensionless coefficients $\alpha_{i}, \gamma_{i}, \delta_{i}$ and $\tilde{\alpha}_{i}, \tilde{\gamma}_{i}, \tilde{\delta}_{i}$ depend on the ratio $\Delta / M$ only. They are detailed in Appendices A and B. The contributions proportional to $\alpha_{i} \delta_{j}$ and $\tilde{\alpha}_{i} \tilde{\delta}_{j}$ in (44) are constructed to ensure that the terms proportional to $\left(m_{Q}^{4} / \Delta\right)$ and $\left(m_{Q}^{4} / \Delta\right) \log m_{Q}^{2}$ are recovered exactly. 
TABLE VII. The loop functions (22) are evaluated with the coupling constants $g_{P}=\tilde{g}_{P} \simeq 0.57$ and the physical isospin-averaged meson masses. A decomposition according to (41) is performed. This leads to (44), (45), (A6), and (B5). All terms are in units of $\mathrm{MeV}$.

\begin{tabular}{lcccc}
\hline \hline$H$ & $\bar{\Pi}_{H}^{\text {bubble }} /\left(2 M_{H}\right)$ & $\bar{\Pi}_{H}^{\text {bubble-3 }} /\left(2 M_{H}\right)$ & $\bar{\Pi}_{H}^{\text {bubble-4 }} /\left(2 M_{H}\right)$ & $\bar{\Pi}_{H}^{\text {bubble }-5} /\left(2 M_{H}\right)$ \\
\hline$D$ & -50.2 & -48.5 & -2.8 & 1.1 \\
$D_{s}$ & -65.6 & -88.3 & 20.1 & 2.9 \\
$D^{*}$ & -113.4 & -99.5 & -17.1 & 3.1 \\
$D_{s}^{*}$ & -166.1 & -197.5 & 26.3 & 6.6 \\
\hline \hline
\end{tabular}

We advance to the fourth-order terms. The explicit expressions are obtained,

$$
\begin{aligned}
\bar{\Pi}_{H \in\left[0^{-}\right]}^{\text {bubble- }}= & \sum_{Q \in[8], R \in\left[1^{-}\right]}\left(\frac{G_{Q R}^{(H)}}{4 \pi f}\right)^{2}\left\{-\frac{1}{4} \alpha_{1} M^{2} \delta_{6}+\frac{M_{H}}{4}\left[\alpha_{1} \Delta^{2} \frac{\partial \Delta \gamma_{1}}{\partial \Delta}+\Delta_{Q}^{2} \beta_{4}-\Delta_{Q}^{2} \beta_{5} \frac{M_{R}-M_{H}}{\Delta_{H}}\right.\right. \\
& -\frac{\beta_{1}}{\Delta_{H}}\left(\left(M_{R}-M_{H}\right)\left(\Delta_{Q}^{2}-\frac{m_{Q}^{2}}{2}\right) \log \frac{m_{Q}^{2}}{M_{R}^{2}}+\Delta_{Q}^{3}\left[\log \left(M_{R}-M_{H}+\Delta_{Q}\right)-\log \left(M_{R}-M_{H}-\Delta_{Q}\right)\right]\right) \\
& \left.\left.+\frac{m_{Q}^{2}}{\Delta_{H}^{2}}\left(-\beta_{2} \Delta_{Q}^{2}+\beta_{3} m_{Q}^{2} \log \frac{m_{Q}^{2}}{M_{R}^{2}}\right)\right]\right\}\left(M_{R}-M_{H}-\Delta_{H}\right), \\
\bar{\Pi}_{H \in\left[1^{-}\right]}^{\text {buble-4 }}= & \sum_{\substack{Q \in[8] \\
R \in\left[1^{-}\right]}}\left(\frac{G_{Q R}^{(H)}}{4 \pi f}\right)^{2} \frac{M_{H}}{3}\left\{\left(-\frac{3 \pi}{4} \frac{m_{Q}}{M_{H}}+1+\frac{1}{2} \log \frac{m_{Q}^{2}}{M_{R}^{2}}\right)\left(m_{Q}^{2}-\left(M_{R}-M_{H}\right)^{2}\right)+\frac{m_{Q}^{2}}{4} \log \frac{m_{Q}^{2}}{M_{R}^{2}}\right\}\left(M_{R}-M_{H}\right) \\
& +\sum_{\substack{Q \in\left[8 \\
R \in 0^{-}\right]}}\left(\frac{G_{Q R}^{(H)}}{4 \pi f}\right)^{2}\left\{\frac{1}{12} \tilde{\alpha}_{1} M \Delta^{2} \tilde{\delta}_{6}+\frac{M_{H}}{12}\left[-\tilde{\alpha}_{1} \Delta^{2} \frac{\partial \Delta \tilde{\gamma}_{1}}{\partial \Delta}-\tilde{\beta}_{4} \Delta_{Q}^{2}+\tilde{\beta}_{5} \Delta_{Q}^{2} \frac{M_{H}-M_{R}}{\Delta_{H}}\right.\right. \\
& -\frac{\tilde{\beta}_{1}}{\Delta_{H}}\left(\left(M_{H}-M_{R}\right)\left(\Delta_{Q}^{2}-\frac{m_{Q}^{2}}{2}\right) \log \frac{m_{Q}^{2}}{M_{R}^{2}}+\Delta_{Q}^{3}\left[\log \left(M_{R}-M_{H}-\Delta_{Q}\right)-\log \left(M_{R}-M_{H}+\Delta_{Q}\right)\right]\right) \\
& \left.\left.-\frac{m_{Q}^{2}}{\Delta_{H}^{2}}\left(-\tilde{\beta}_{2} \Delta_{Q}^{2}+\tilde{\beta}_{3} m_{Q}^{2} \log \frac{m_{Q}^{2}}{M_{R}^{2}}\right)\right]\right\}\left(M_{R}-M_{H}+\Delta_{H}\right),
\end{aligned}
$$

with $\Delta_{Q}$ and $\Delta_{H}$ already introduced in (44).
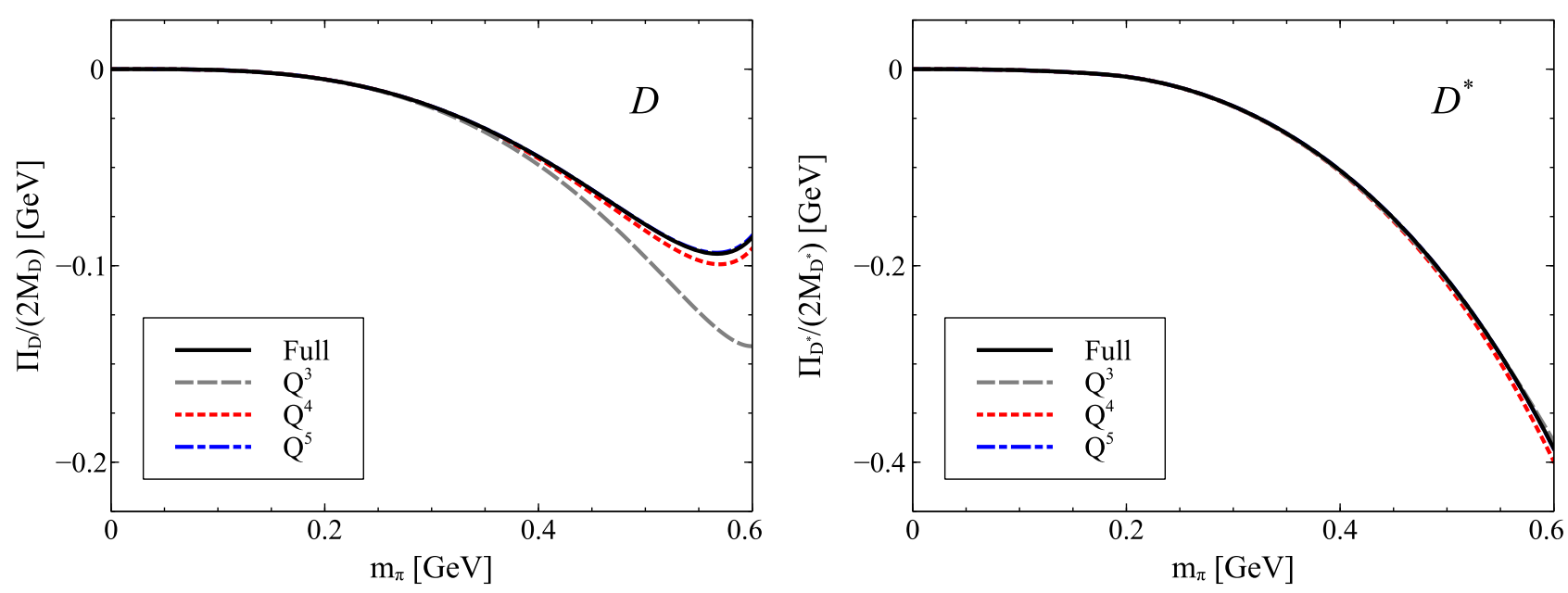

FIG. 4. D-meson masses in the flavor limit as a function of the pion mass. The power-counting decomposed loop functions of (44), (45), (A6), and (B5) are used with the parameter set of Table IV. 
In Table VII, we decompose the loop function into third-, fourth-, and fifth-order numerical values. The results are compared with the exact numbers already shown in Table IV. The conclusions of that table are unambiguous: the power-counting ansatz (41) is well justified for a chiral extrapolation of the $D$-meson masses. We note that the fifth-order contributions to the $D$-meson masses are on average about $3 \mathrm{MeV}$ only. Our novel expansion scheme is characterized by a rapid convergence property. All $D$-meson masses are reproduced at the few $\mathrm{MeV}$ level. We further substantiate our claim by Fig. 4, which shows the loop function $\bar{\Pi}_{H}$ in the flavor limit with $m_{\pi}=m_{K}=m_{\eta}$. The figures are in correspondence to Figs. 1, 2, and 3 and demonstrate that for any reasonable pion mass, say, $0 \leq m_{\pi}<600 \mathrm{MeV}$, a quantitative reproduction of the solid line is obtained. We conclude that it is justified to identify the full loop expressions as the loop function to be used at chiral order $Q^{4}$ without any significant error from the incomplete fifth-order terms.

\section{FIT TO QCD LATTICE DATA}

In this section, we will determine the low-energy constants $c_{i}$ and $d_{i}$ of the chiral Lagrangian from lattice QCD simulations of the $D$-meson masses. Open-charm mesons have been extensively studied on different QCD lattices [10,18, 20-22,46-54]. For a recent review, we refer to Ref. [55]. There exists a significant data set for $D$-meson masses at various unphysical quark masses. We consider data sets in which the pion and kaon masses are smaller than about $600 \mathrm{MeV}$ only. Once we determined the low-energy constants (LECs) in our mass formula, the $D$-meson masses can be computed at any values for the up-, down-, and strange-quark masses, sufficiently small to justify the application of the chiral extrapolation.

Though in principle such an analysis can be done at different chiral orders, we do so using the subtracted loop expressions (22) in (28) with the scalar loop functions as worked out previously for the finite box case in Ref. [34]. It is a matter of convenience to perform our fits using the full one-loop functions rather than any truncated form. Therewith, the finite volume corrections specific to the various chiral moments, the explicit derivation of which would require further tedious algebra, are not required. This strategy is justified since we have demonstrated with Table VII that the full loop function is reproduced quite accurately by its $\mathrm{N}^{3} \mathrm{LO}$ approximation, with a residual uncertainty for the $D$-meson masses of about $3 \mathrm{MeV}$ only. It is emphasized that such a point of view relies heavily on our reorganized chiral expansion approach, which is formulated in terms of physical meson masses.

While for instance in Refs. [18,20] the extrapolation toward the physical point was the focus, the purpose of our study is the extraction of the low-energy constants of the chiral Lagrangian. Therefore, a different strategy is used in our work. We use the empirical $D$-meson masses as an additional constraint in our analysis. For a given pion and kaon mass, we infer the quark masses from the one-loop mass formulas for the pseudo-Goldstone bosons to be used in our expressions for the $D$-meson masses. Assuming that the lattice data can be properly moved to the physical charm-quark mass, the low-energy constants are obtained by a global fit to the QCD lattice data set. Altogether, there are about 80 data points considered in our analysis.

A comprehensive published data set is from Mohler and Woloshyn [18,53] based on the PACS-CS ensembles [17]. The Fermilab approach is employed in implementing the valence charm quark [56,57]. In this approach, heavy quark mass-dependent counterterms are added in the heavy-quark action to systematically reduce discretization effects. The valence charm quark-mass dependence is parametrized by a hopping parameter $\kappa_{c}$, which is tuned to match the average of the physical kinematic $D$-meson masses. In Table VIII, we recall the relevant results, which are the pion, kaon, and the four $D$-meson masses in units of the lattice spacing $a$. The levels for the $D$ mesons as given in Table VIII are not the masses but rather energies measured relative to some fixed reference. In turn, only mass differences of $D$ mesons are constrained by that table in our studies.

Recently, the group of Marc Wagner analyzed a large set of ensembles from the ETMC [20,21]. Our analysis requires the $D$-meson masses evaluated at the physical charm-quark mass. We are grateful to the authors of Ref. [20] for making available unpublished results, which allow us to independently extrapolate their lattice data to the physical charm-quark mass. For each ensemble, the four $D$-meson masses and also the $\eta_{c}$ and $J / \Psi$ masses are computed at two different values of the charm

TABLE VIII. Meson masses and energy levels in units of the lattice spacing $a$ as taken from Refs. [18,53,17]. Statistical errors are given only. The results are based on ensembles from PACS for which their estimate of the lattice spacing is $a=0.0907(13) \mathrm{fm}$.

\begin{tabular}{lllllll}
\hline \hline & \multicolumn{1}{c}{$a m_{\pi}$} & \multicolumn{1}{c}{$a m_{K}$} & \multicolumn{1}{c}{$a E_{D}$} & \multicolumn{1}{c}{$a E_{D_{s}}$} & $a E_{D^{*}}$ & $a E_{D_{s}^{*}}$ \\
\hline $32^{3} \times 64$ & $0.0717(32)$ & $0.2317(6)$ & $0.7765(12)$ & $0.8197(24)$ & $0.8447(27)$ & $0.8850(24)$ \\
$32^{3} \times 64$ & $0.13593(140)$ & $0.27282(103)$ & $0.78798(82)$ & $0.83929(26)$ & $0.85776(122)$ & $0.90429(43)$ \\
$32^{3} \times 64$ & $0.17671(129)$ & $0.26729(110)$ & $\ldots$ & $0.82848(40)$ & $\ldots$ & $0.89015(69)$ \\
$32^{3} \times 64$ & $0.18903(79)$ & $0.29190(67)$ & $0.79580(61)$ & $0.84000(36)$ & $0.86327(99)$ & $0.90429(60)$ \\
\hline \hline
\end{tabular}


TABLE IX. Meson masses in units of the lattice spacing $a$ based on the ensembles of the ETM Collaboration. The values in the table are provided to us by the authors of Ref. [20]. Statistical errors are given only. The data correspond to three different $\beta_{\mathrm{QCD}}=1.90,1.95,2.10$ values for which in Ref. [58] an estimate of the lattice scale is provided with $a=0.0934(37), 0.0820(37), 0.0644(26)$ fm, respectively.

\begin{tabular}{|c|c|c|c|c|c|c|}
\hline & $a(\mathrm{fm})$ & $a m_{\pi}$ & $a m_{K}$ & $a \mu_{c}$ & $a M_{\eta_{c}}$ & $a M_{J / \Psi}$ \\
\hline \multirow[t]{2}{*}{$48^{3} \times 96$} & 0.0619 & $0.0703(4)$ & $0.1697(3)$ & 0.2230 & $1.0595(2)$ & $1.1006(3)$ \\
\hline & & & & 0.1919 & $0.9570(2)$ & $1.0003(4)$ \\
\hline \multirow[t]{2}{*}{$48^{3} \times 96$} & 0.0619 & $0.0806(3)$ & $0.1738(5)$ & 0.2227 & $1.0579(2)$ & $1.0989(4)$ \\
\hline & & & & 0.1727 & $0.8915(2)$ & $0.9364(5)$ \\
\hline \multirow[t]{2}{*}{$48^{3} \times 96$} & 0.0619 & $0.0975(3)$ & $0.1768(3)$ & 0.2230 & $1.0591(1)$ & $1.1002(3)$ \\
\hline & & & & 0.1727 & $0.8919(1)$ & $0.9370(3)$ \\
\hline \multirow[t]{2}{*}{$32^{3} \times 64$} & 0.0815 & $0.1074(5)$ & $0.2133(4)$ & 0.2230 & $1.3194(2)$ & $1.3835(4)$ \\
\hline & & & & 0.1727 & $1.1567(2)$ & $1.2233(4)$ \\
\hline \multirow[t]{2}{*}{$32^{3} \times 64$} & 0.0815 & $0.1549(2)$ & $0.2279(2)$ & 0.2230 & $1.3251(1)$ & $1.3903(2)$ \\
\hline & & & & 0.1727 & $1.1573(1)$ & $1.2253(2)$ \\
\hline \multirow[t]{2}{*}{$24^{3} \times 48$} & 0.0815 & $0.1935(4)$ & $0.2430(4)$ & 0.2230 & $1.3179(3)$ & $1.3837(4)$ \\
\hline & & & & 0.1727 & $1.1582(3)$ & $1.2273(4)$ \\
\hline \multirow[t]{2}{*}{$32^{3} \times 64$} & 0.0885 & $0.1240(4)$ & $0.2512(3)$ & 0.2772 & $1.3869(1)$ & $1.4649(3)$ \\
\hline & & & & 0.2270 & $1.2241(2)$ & $1.3042(4)$ \\
\hline \multirow[t]{2}{*}{$32^{3} \times 64$} & 0.0885 & $0.1412(3)$ & $0.2569(3)$ & 0.2768 & $1.3859(1)$ & $1.4636(3)$ \\
\hline & & & & 0.2389 & $1.2642(1)$ & $1.3430(3)$ \\
\hline \multirow[t]{2}{*}{$24^{3} \times 48$} & 0.0885 & $0.1440(6)$ & $0.2589(4)$ & 0.2768 & $1.3863(2)$ & $1.4645(4)$ \\
\hline & & & & 0.2389 & $1.2645(2)$ & $1.3442(5)$ \\
\hline \multirow[t]{2}{*}{$24^{3} \times 48$} & 0.0885 & $0.1988(3)$ & $0.2764(3)$ & 0.2929 & $1.4273(2)$ & $1.5069(4)$ \\
\hline & & & & 0.2299 & $1.2353(2)$ & $1.3172(5)$ \\
\hline
\end{tabular}

valence-quark mass $\mu_{c}$. As a consequence of the discretization procedure, there are corresponding pairs of meson masses that turn degenerate in the continuum limit. We use the notation $( \pm, \mp)$ and $( \pm, \pm)$ from Refs. [20,21]. In this work, we focus on the $( \pm, \mp)$ states and use the masses of the partner states $( \pm, \pm)$ only as a rough estimate for the size of the discretization error. In the vicinity of the physical charm-quark mass, a linear behavior,

$$
a M_{H}=\alpha_{H}+\beta_{H} a \mu_{c},
$$

is expected to hold for all hadron masses. Since the chosen charm-quark masses are close to the physical one, the ansatz (46) should be justified to sufficient accuracy. The parameters $\alpha_{H}$ and $\beta_{H}$ can be extracted from the data provided to us by Kalinowski and Wagner. In Table IX, we show their results for the $\eta_{c}$ and $J / \Psi$ masses together with their preferred lattice spacing values $a$. Corresponding results for the $D$-meson masses are listed at the end of Appendix B. The task of determining the physical value for $\mu_{c}$ remains. Since one would not expect a significant dependence of the $\eta_{c^{-}}$nor the $J / \psi$-meson mass on the precise value of the up-, down-, and strange-quark masses, one may contemplate using either of the two masses to obtain a good estimate for $\mu_{c}$. Both scenarios are scrutinized in the following based on the data of Kalinowski and Wagner. To fix the charm-quark mass, we always choose the ensemble with the lightest up- and down-quark masses.
In addition, the lattice spacing $a$ as recalled in Table IX is assumed. A typical example for this procedure is shown in Fig. 5, in which a sizable uncertainty for the extracted value of $\mu_{c}$ is observed.

How such an uncertainty propagates into the masses of the $D$ mesons is shown in Tables XI and X, which are based on the charm-quark masses from the $\eta_{c}$ and the $J / \psi$ mesons, respectively. As expected, this uncertainty in the charm-quark mass is reduced for the ensembles that correspond to even smaller lattice spacings with $a=$ $0.0815 \mathrm{fm}$ and $a=0.0619 \mathrm{fm}$. This can be inferred by a

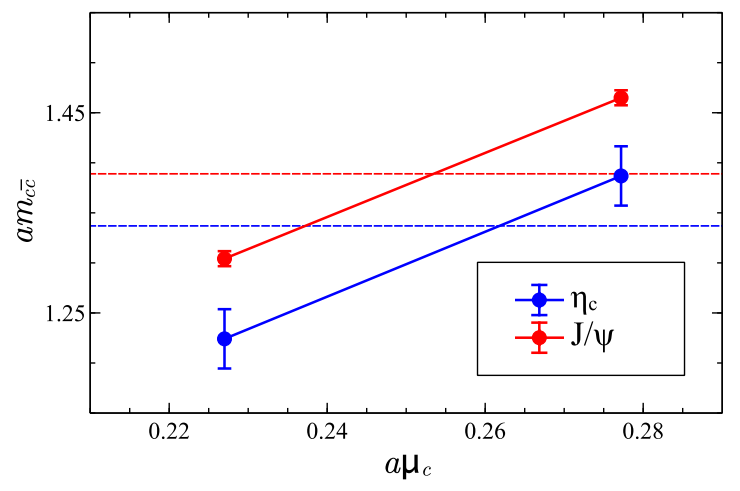

FIG. 5. The interpolation of charmonium masses to determine $\mu_{c}$, at given $a=0.0885 \mathrm{fm}$. The ensemble is chosen with $a m_{\pi}=0.1240$. The physical values of $a M_{\eta_{c}}$ and $a M_{J / \psi}$ are indicated by the dashed lines. 
TABLE X. $D$ - and $J / \psi$-meson masses in units of the lattice scale $a$. The charm-quark mass is determined to reproduce the physical $J / \psi$ mass. This leads to $a \mu_{c}=0.2535,0.1902$, and 0.1829 for the three groups of ensembles. Statistical errors are given only.

\begin{tabular}{lcccccc}
\hline \hline$a m_{\pi}$ & $a m_{K}$ & $a M_{D}$ & $a M_{D_{s}}$ & $a M_{D^{*}}$ & $a M_{D_{s}^{*}}$ & $a M_{J / \psi}$ \\
\hline $0.0703(4)$ & $0.1697(3)$ & $0.5905(52)$ & $0.6236(56)$ & $0.6466(86)$ & $0.6770(28)$ & $0.9715(20)$ \\
$0.0806(3)$ & $0.1738(5)$ & $0.5906(64)$ & $0.6234(57)$ & $0.6506(26)$ & $0.6763(11)$ & $0.9697(21)$ \\
$0.0975(3)$ & $0.1768(3)$ & $0.5913(50)$ & $0.6229(57)$ & $0.6486(28)$ & $0.6764(15)$ & $0.9703(21)$ \\
$0.1074(5)$ & $0.2133(4)$ & $0.7840(122)$ & $0.8159(147)$ & $0.8568(44)$ & $0.8905(34)$ & $1.2791(55)$ \\
$0.1549(2)$ & $0.2279(2)$ & $0.7895(128)$ & $0.8183(144)$ & $0.8678(47)$ & $0.8950(39)$ & $1.2828(55)$ \\
$0.1935(4)$ & $0.2430(4)$ & $0.7934(148)$ & $0.8175(151)$ & $0.8745(38)$ & $0.8965(41)$ & $1.2818(58)$ \\
$0.1240(4)$ & $0.2512(3)$ & $0.8514(181)$ & $0.8953(206)$ & $0.9356(28)$ & $0.9806(45)$ & $1.3890(75)$ \\
$0.1412(3)$ & $0.2569(3)$ & $0.8544(168)$ & $0.8972(208)$ & $0.9363(41)$ & $0.9802(45)$ & $1.3895(75)$ \\
$0.1440(6)$ & $0.2589(4)$ & $0.8552(159)$ & $0.8978(208)$ & $0.9403(23)$ & $0.9844(45)$ & $1.3906(77)$ \\
$0.1988(3)$ & $0.2764(3)$ & $0.8599(184)$ & $0.8950(219)$ & $0.9487(60)$ & $0.9841(66)$ & $1.3882(79)$ \\
\hline \hline
\end{tabular}

TABLE XI. $D$ - and $\eta_{c}$-meson masses in units of the lattice scale $a$. The charm-quark mass was determined to reproduce the physical $\eta_{c}$ meson mass. This leads to $a \mu_{c}=0.2618,0.1957$, and 0.1852 for the three groups of ensembles. Statistical errors are given only.

\begin{tabular}{lcccccc}
\hline \hline$a m_{\pi}$ & $a m_{K}$ & $a M_{D}$ & $a M_{D_{s}}$ & $a M_{D^{*}}$ & $a M_{D_{s}^{*}}$ & $a M_{\eta_{c}}$ \\
\hline $0.0703(4)$ & $0.1697(3)$ & $0.5947(52)$ & $0.6279(56)$ & $0.6506(86)$ & $0.6809(28)$ & $0.9351(85)$ \\
$0.0806(3)$ & $0.1738(5)$ & $0.5949(64)$ & $0.6277(57)$ & $0.6546(26)$ & $0.6803(11)$ & $0.9332(85)$ \\
$0.0975(3)$ & $0.1768(3)$ & $0.5955(50)$ & $0.6271(57)$ & $0.6526(28)$ & $0.6804(15)$ & $0.9335(84)$ \\
$0.1074(5)$ & $0.2133(4)$ & $0.7946(122)$ & $0.8263(147)$ & $0.8664(44)$ & $0.9001(34)$ & $1.2312(212)$ \\
$0.1549(2)$ & $0.2279(2)$ & $0.8004(128)$ & $0.8291(144)$ & $0.8777(47)$ & $0.9049(39)$ & $1.2342(217)$ \\
$0.1935(4)$ & $0.2430(4)$ & $0.8039(148)$ & $0.8278(151)$ & $0.8840(38)$ & $0.9059(41)$ & $1.2314(219)$ \\
$0.1240(4)$ & $0.2512(3)$ & $0.8677(181)$ & $0.9114(206)$ & $0.9506(28)$ & $0.9953(45)$ & $1.3370(296)$ \\
$0.1412(3)$ & $0.2569(3)$ & $0.8708(168)$ & $0.9132(208)$ & $0.9511(41)$ & $0.9949(45)$ & $1.3379(299)$ \\
$0.1440(6)$ & $0.2589(4)$ & $0.8714(159)$ & $0.9137(208)$ & $0.9545(24)$ & $0.9990(45)$ & $1.3382(302)$ \\
$0.1988(3)$ & $0.2764(3)$ & $0.8753(184)$ & $0.9102(219)$ & $0.9627(60)$ & $0.9980(66)$ & $1.3325(310)$ \\
\hline \hline
\end{tabular}

comparison of Tables XI and X. While the center values of the masses in Tables XI and $\mathrm{X}$ are derived from the $( \pm, \mp)$ states of Tables XXI and XXII in Appendix B, the shown error bars entail an estimate for the total error including the statistical error and the uncertainty from the discretization procedure. We take half of the splittings of the two modes, $( \pm, \mp)$ and $( \pm, \pm)$, for the latter.

It is immediate from Tables XI and X that the $D$-meson masses are quite sensitive to the precise charm-quark mass used and also to the lattice scale $a$ assumed. We note that, for instance, there exist two distinct values for the lattice spacing for the coarsest ensembles: the value $a=0.0885(36) \mathrm{fm}$ obtained from the pion decay constant [59] and $a=$ $0.0920(21) \mathrm{fm}$ obtained from the nucleon mass [58]. We conclude that it may advantageous to determine the lattice scale and the charm-quark mass from the $D$-meson masses directly. Such a procedure is expected to minimize the discretization errors for the $D$-meson masses. This is what we will do in the following. All information required for such a strategy is provided with Tables XI and X, from which the parameters $\alpha_{H}$ and $\beta_{H}$ in (46) can be read off.
There are yet three further sources of QCD lattice data on the $D$-meson masses, which we will discuss briefly $[10,19,22]$. The two data sources $[10,19]$ are partial to the extent that not all four $D$-meson masses are provided. Only the pseudoscalar masses are computed. The results of Ref. [10] rely on previous studies by the LHP Collaboration [60], which uses a mixed action framework with domainwall valence quarks but staggered sea-quark ensembles generated by MILC [61-65]. For the charm quark, it uses a relativistic heavy-quark action motivated by the Fermilab approach [56,57]. In Table XII, we summarize the relevant masses that are considered in our study.

The results of the HPQCD Collaboration [19] are based on MILC ensembles together with a highly improved staggered valence quark (HISQ) action. The HISQ action has since been used very successfully in simulations involving the charm quark such as for charmonium and for $D$ - and $D_{s}$-meson decay constants. In Table XIII, we collect the relevant masses in units of the lattice spacing for the configurations on three coarse and two fine lattices. 
TABLE XII. $D$-meson masses based on ensembles of MILC [63] as used by LHPC [60]. The results are recalled from Ref. [10] in units of the lattice spacing $a$. The lattice spacing is $a \simeq 0.12 \mathrm{fm}$.

\begin{tabular}{ccccc}
\hline \hline & $a m_{\pi}$ & $a m_{K}$ & $a M_{D}$ & $a M_{D_{s}}$ \\
\hline $20^{3} \times 64$ & $0.1842(7)$ & $0.3682(5)$ & $1.2081(13)$ & $1.2637(10)$ \\
$20^{3} \times 64$ & $0.2238(5)$ & $0.3791(5)$ & $1.2083(11)$ & $1.2635(10)$ \\
$20^{3} \times 64$ & $0.3113(4)$ & $0.4058(4)$ & $1.2226(13)$ & $1.2614(12)$ \\
$20^{3} \times 64$ & $0.3752(5)$ & $0.4311(5)$ & $1.2320(11)$ & $1.2599(12)$ \\
\hline \hline
\end{tabular}

TABLE XIII. $D$-meson masses from the HPQCD Collaboration in units of the lattice spacing $a$ as taken from Refs. [19,47]. The studies are based on ensembles of MILC [65]. The lattice spacings are $a=0.119(2) \mathrm{fm}$ and $a=0.0846(7) \mathrm{fm}$ for the two sets of data, respectively.

\begin{tabular}{ccccc}
\hline \hline & $a m_{\pi}$ & $a m_{K}$ & $a M_{D}$ & $a M_{D_{s}}$ \\
\hline $24^{3} \times 64$ & $0.1599(2)$ & $0.3122(2)$ & $1.1395(7)$ & $1.1878(3)$ \\
$20^{3} \times 64$ & $0.2108(2)$ & $0.3285(3)$ & $1.1591(7)$ & $1.2014(4)$ \\
$20^{3} \times 64$ & $0.2931(2)$ & $0.3572(2)$ & $1.1618(5)$ & $1.1897(3)$ \\
$28^{3} \times 96$ & $0.1344(2)$ & $0.2286(2)$ & $0.8130(3)$ & $0.8471(2)$ \\
$28^{3} \times 96$ & $0.1873(1)$ & $0.2458(2)$ & $0.8189(3)$ & $0.8434(2)$ \\
\hline \hline
\end{tabular}

Most recently, the HSC computed the excited open-charm meson spectrum in a finite QCD box [22,23]. Results for the $D$-meson masses based on an ensemble with a pion mass of about $390 \mathrm{MeV}$ are published in Ref. [23] and recalled in Table XIV. For an additional ensemble at smaller pion masses, studies are ongoing [22].

We note that the charm-quark mass in Refs. [10,19,23] was not adjusted to the $D$-meson masses. While in Ref. [10] the spin average of the physical $J / \Psi$ - and $\eta_{c}$-meson mass was used, and in Ref. [19], the charm-quark mass was tuned to the physical $\eta_{c}$ mass. In both cases, we cannot exclude uncertainties significant to our analysis. To minimize any bias from a possibly imprecise charm quarkmass determination, we consider only mass differences from Tables XII-XIV in our fits. In addition, we fine tune the lattice scales. As we have seen in the case of the ETMC results, such a procedure reduces any possible bias significantly.

We introduce a universal parameter $\Delta_{c}$ of the form

$$
a M_{H} \rightarrow a M_{H}+\left(1+\epsilon_{H}\right) a \Delta_{c}, \quad \text { with } \quad \epsilon_{H} \simeq 0,
$$

which is supposed to fine tune the choice of the charmquark mass. In principle, the values of $\epsilon_{H}$ depend on the type of the $D$ meson considered and also the $\beta_{\mathrm{QCD}}$ value of the ensemble considered. The value $\left(1+\epsilon_{H}\right) a \Delta_{c}$ is to be added to $a M_{H}$ as collected in Tables XI-XIV.

For the ETMC masses, the magnitude of $\epsilon_{H}$ can be extracted from Tables X and XI, in which we insist on the normalization condition that $\epsilon_{H}=0$ for the $D$ meson on the ensemble with the lightest pion mass. Then, values for $\left|\epsilon_{H}\right|$ of about 0.1 arise in some cases at most. Such an estimate is not available for the other collaborations. For these other cases, we put $\epsilon_{H}=0$, which would arise in the heavy quark-mass limit. We would argue that a precise determination of $a \Delta_{c}$ and therewith the physical charm-quark mass for a given ensemble requires the quantitative control of the chiral extrapolation formulas for the $D$-meson masses.

We do not implement discretization effects in our chiral extrapolation approach since this would introduce a significant number of further unknown parameters into the game. For each lattice group, such effects have to be worked out in the context of our chiral extrapolation scheme. As a consequence, a fully systematic error analysis is not possible yet in our present study. Here, we follow the strategy suggested in Refs [34,35], in which the statistical error given by the lattice groups is supplemented by a systematic error in mean quadrature. We perform fits at different ad hoc values for the systematic error. Once this error is sufficiently large, the $\chi^{2}$ per data point should be close to 1 . In our current studies, we arrive at the estimate of 5-10 MeV. In anticipation of our analysis of the lattice data set, we collect the result of four representative fits. Their characteristics and defining assumptions will be discussed in more detail in the next sections.

For a given ensemble, the statistical errors in the lattice data are correlated. However, since the statistical error for any meson mass considered here is typically much smaller than our estimate for the systematic error, such a correlation is of no relevance in our study. In contrast, the choice of the charm-quark mass and the lattice scale setting, both of which we treat in detail, is a significant effect.

Our fit procedure goes as follows. For a given lattice ensemble, we take the pion and kaon masses as given in lattice units and then determine from the one-loop expressions (28) in Ref. [35] the quark masses for that ensemble. They depend on the three particular linear combinations of the low-energy constants of Gasser and Leutwyler [67]. One combination can be fixed by the request that the

TABLE XIV. $D$-meson masses from HSC in units of the temporal lattice spacing $[23,66]$. The lattice spacing is $3.5 a_{t}=0.123(4) \mathrm{fm}$. It holds that $a=a_{s} \simeq 3.5 a_{t}$.

\begin{tabular}{llllllc}
\hline \hline & $a_{t} m_{\pi}$ & $a_{t} m_{K}$ & $a_{t} M_{D}$ & $a_{t} M_{D_{s}}$ & $a_{t} M_{D^{*}}$ & $a_{t} M_{D_{s}^{*}}$ \\
\hline $24^{3} \times 128$ & $0.06906(13)$ & $0.09698(9)$ & $0.33265(7)$ & $0.34426(6)$ & $0.35415(17)$ & $0.36508(88)$ \\
$32^{3} \times 256$ & $0.03928(18)$ & $0.08344(7)$ & $\cdots$ & $\cdots$ & $\cdots$ & $\cdots$ \\
\hline \hline
\end{tabular}


TABLE XV. Results for fit 1-fit 4. The low-energy constants $L_{n}$ are at the renormalization scale $\mu=0.77 \mathrm{GeV}$. The offset parameters $a \Delta_{c}$ are introduced in (47). We use $f=92.4 \mathrm{MeV}$ throughout this work. A more detailed discussion of the four fit scenarios is given in Secs. VII and VIII.

\begin{tabular}{|c|c|c|c|c|}
\hline & Fit 1 & Fit 2 & Fit 3 & Fit 4 \\
\hline$a_{\text {PACS-CS}}(\mathrm{fm})$ & 0.0934 & 0.0940 & 0.0935 & 0.0928 \\
\hline$a \Delta_{c, \mathrm{PACS}-\mathrm{CS}}$ & 0.1067 & 0.1110 & 0.1119 & 0.1023 \\
\hline$a_{\text {LHPC }}(\mathrm{fm})$ & 0.1291 & 0.1267 & 0.1291 & 0.1291 \\
\hline$a \Delta_{c, \mathrm{LHPC}}$ & 0.0359 & 0.0087 & 0.0443 & 0.0381 \\
\hline$a_{c, \mathrm{HPOCD}}^{\beta \simeq 6.76}(\mathrm{fm})$ & 0.1367 & 0.1359 & 0.1336 & 0.1367 \\
\hline$a \Delta_{c . \mathrm{HPOCD}}^{\beta \simeq 6.76}$ & 0.1500 & 0.1494 & 0.1184 & 0.1500 \\
\hline$a_{c, \mathrm{HPQCD}}^{\beta \simeq 7.09}(\mathrm{fm})$ & 0.0953 & 0.0991 & 0.0970 & 0.0992 \\
\hline$a \Delta_{c, \mathrm{HPQCD}}^{\beta \simeq 7.09}$ & 0.0936 & 0.1336 & 0.1049 & 0.1282 \\
\hline$a_{c, \text { ETMC }}^{\beta=1.90}(\mathrm{fm})$ & 0.1018 & 0.0996 & 0.1025 & 0.1027 \\
\hline$a \Delta_{c . \mathrm{ETMC}}^{\beta=1.90}$ & 0.0983 & 0.0747 & 0.1041 & 0.1086 \\
\hline$a_{\mathrm{ETMC}}^{\beta=1.95}(\mathrm{fm})$ & 0.0934 & 0.0925 & 0.0928 & 0.0943 \\
\hline$a \Delta_{c, \mathrm{ETMC}}^{\beta=1.95}$ & 0.0908 & 0.0817 & 0.0817 & 0.1005 \\
\hline$a_{\mathrm{ETMC}}^{\beta=2.10}(\mathrm{fm})$ & 0.0695 & 0.0704 & 0.0695 & 0.0699 \\
\hline$a \Delta_{c, \mathrm{ETMC}}^{\beta=2.10}$ & 0.0629 & 0.0728 & 0.0608 & 0.0659 \\
\hline$a_{\mathrm{HSC}}(\mathrm{fm})$ & 0.1211 & 0.1243 & 0.1242 & 0.1242 \\
\hline$a \Delta_{c, \mathrm{HSC}}$ & 0.0050 & 0.0337 & 0.0328 & 0.0343 \\
\hline $10^{3}\left(L_{4}-2 L_{6}\right)$ & -0.1395 & -0.1112 & -0.1102 & -0.1575 \\
\hline $10^{3}\left(L_{5}-2 L_{8}\right)$ & 0.0406 & -0.0940 & -0.0235 & -0.0370 \\
\hline $10^{3}\left(L_{8}+3 L_{7}\right)$ & -0.5130 & -0.5127 & -0.4950 & -0.5207 \\
\hline$m_{s} / m$ & 26.547 & 26.187 & 26.596 & 26.600 \\
\hline
\end{tabular}

$\eta$-meson mass is reproduced at physical quark masses. The other two are determined by our fit to lattice data. With those, the quark-mass ratio $m_{s} / m$ is determined. This is analogous to Ref. [35], in which those low-energy constants are determined from a fit to the lattice data on baryon masses. In Table XV, we show our results for four distinct fit scenarios, which are reasonably close to the results of
Ref. [35]. The quark-mass ratio $m_{s} / m$ as given in the last row of the table is compatible with the latest result of ETMC [59] with $m_{s} / m=26.66(32)$. In Table XV, also, the lattice scale parameters $a$ together with the offset charm quark-mass parameters $\Delta_{c}$ are presented. All fits reproduce the $D$-meson masses of all ensembles recalled in this work quite well. The table illustrates that the offset parameters are almost always non-negligible. Our values for the lattice scale can be compared with the ones advocated by the various lattice groups as recalled in the tables of this section. Any deviation from such values may be viewed as a reflection of significant discretization effects. Those depend on the specifics of the scale setting. The aim of our work is to minimize such discretization effects in the open-charm meson sector of QCD. We find it interesting that, in particular, our values for ETMC are amazingly close to those lattice scales obtained in our previous analysis of the baryon masses from the identical lattice ensembles [35].

The quality of the data description is illustrated with fit 1 , for which we offer a comparison with the lattice data in Figs. 6-8. A more quantitative comparison with $\chi^{2}$ values will be provided in the next section. In all figures, open symbols correspond to results from our chiral extrapolation approach. They lie always on top of the lattice points, which are shown with either green, blue, or red filled symbols. In the case in which for a considered lattice ensemble there is no lattice result for the considered $D$ meson mass available, our theory prediction is presented with a yellow filled symbol.

In Fig. 6, we scrutinize the lattice results of Refs. $[10,18,53]$ as recalled in Tables VIII and XII. Note that the strange-quark mass varies along the different pion masses of the figure. The $D$-meson masses are shown in units of $\mathrm{GeV}$, where the lattice scales for the two groups are taken from Table XV. In addition, the effect of the finetuned charm-quark mass in terms of the appropriate $\Delta_{c}$ values in Table XV is considered. From Fig. 6, we conclude that all masses from Refs. $[10,18,53]$ are recovered well

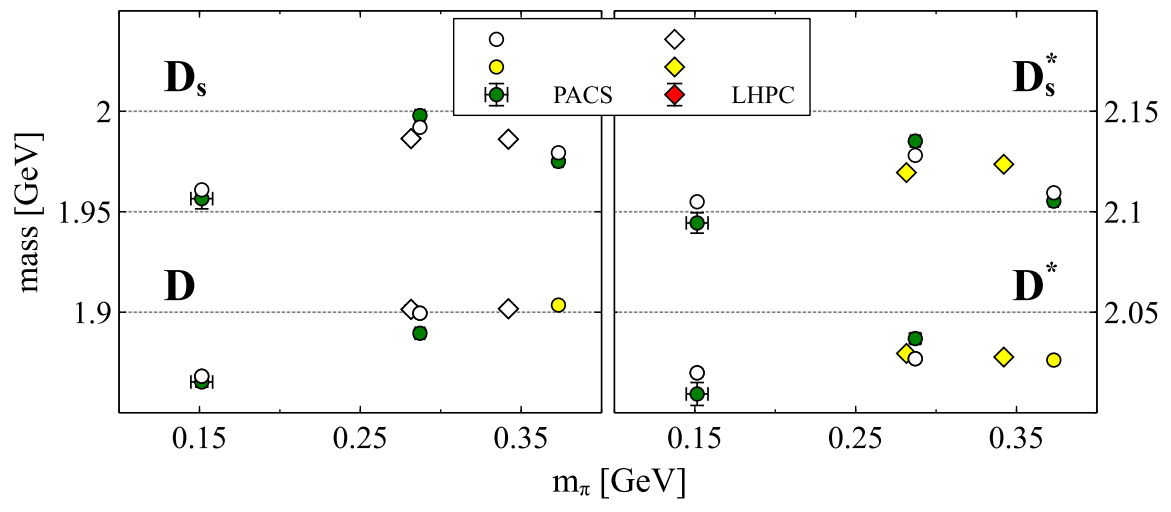

FIG. 6. D-meson masses from fit 1 compared to results based on lattice ensembles from PACS-CS and LHPC [10,18,53]. The yellow symbols present our predictions for the case in which no lattice values are available yet. 


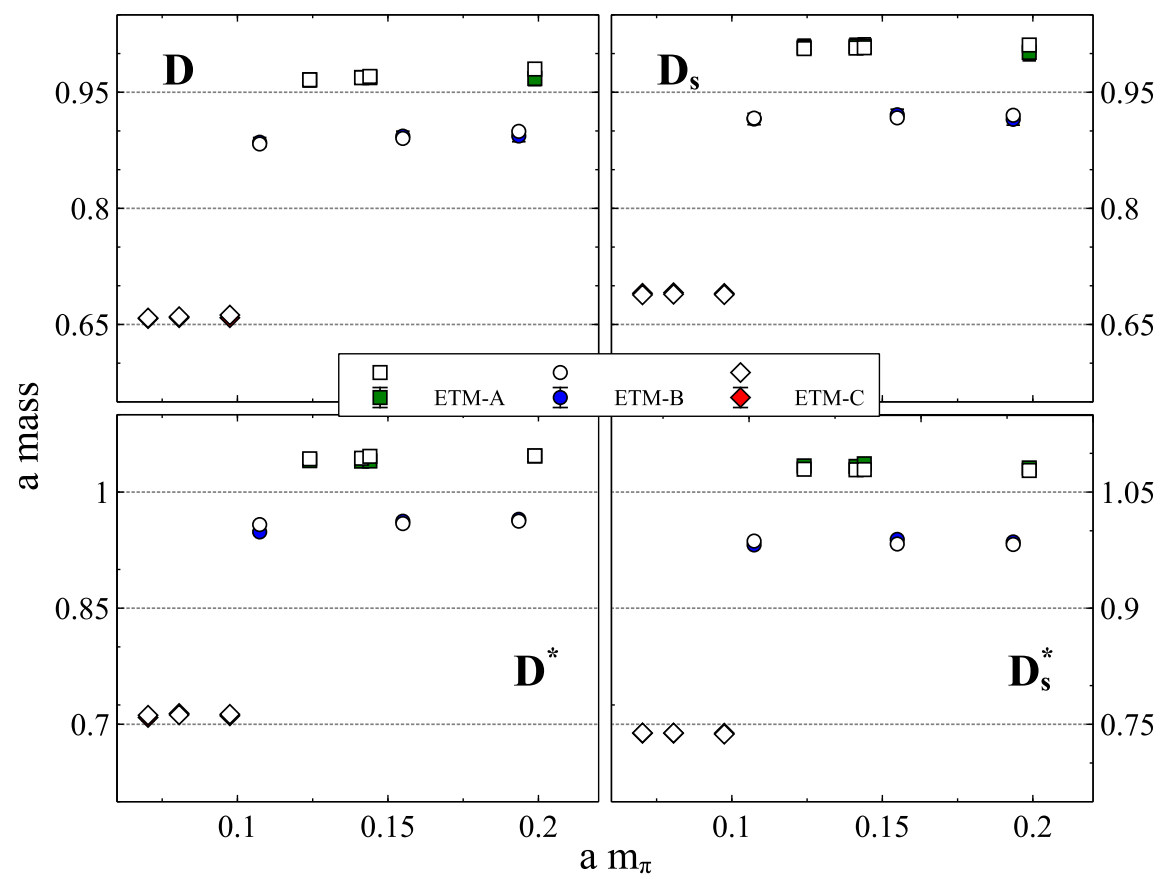

FIG. 7. $D$-meson masses from fit 1 in lattice units as compared to results from ETMC [20].

with an uncertainty of less than $10 \mathrm{MeV}$. The figures include predictions of five meson masses shown with yellow symbols for which there do not exist so far corresponding values from the lattice collaborations. Note that in some cases the lattice data point is fully covered with our chiral extrapolation symbol. This signals an almost perfect reproduction of the lattice point.

We continue with Fig. 7, in which the predictions of ETMC are compared to our results. Here, the meson masses are shown in lattice units. This permits an efficient

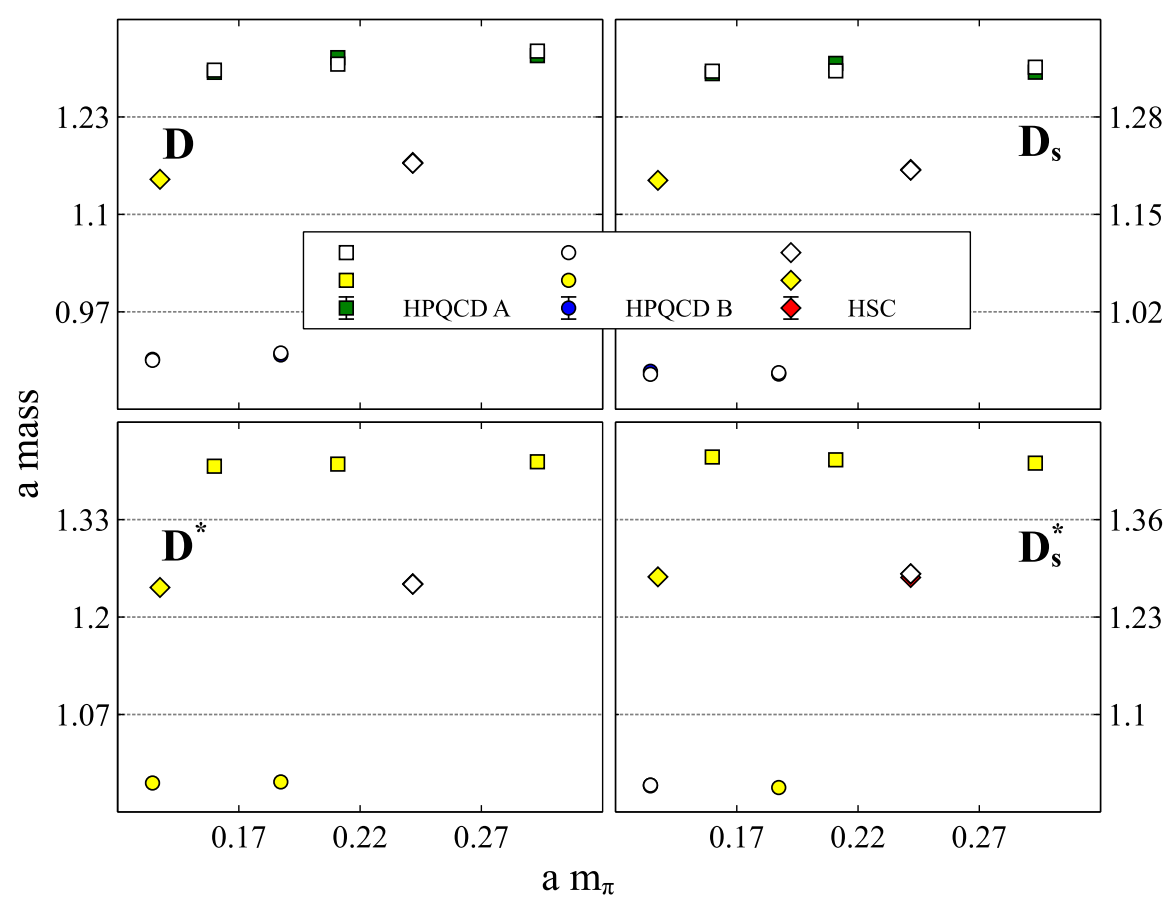

FIG. 8. $D$-meson masses from fit 1 in lattice units as compared to results from HPQCD and HSC $[19,23,47]$. The yellow symbols present our predictions for the case in which no lattice values are available yet. Note that we show the HPQCD data in units of their spatial lattice spacing but the HSC data in units of 3.5 times their temporal lattice spacing. 
presentation of the results at three distinct $\beta_{\mathrm{QCD}}$ values. The data set of ETMC is of particular importance for the chiral extrapolation since it offers masses for the $J^{P}=0^{-}$and $J^{P}=1^{-}$states consistently. The figure illustrates that such data can be reproduced accurately for all $\beta_{\mathrm{QCD}}$ values. Note that the effect of a fine-tuned charm-quark mass is considered again in terms of the parameter $\Delta_{c}$ properly taken from Table XV.

A discussion of Fig. 8, which combines results from HPQCD and HSC [19,23,47], remains. Again, the meson masses are shown in lattice units with $\Delta_{c}$ from Table XV. The reproduction of the lattice data is again impressive. The reader is pointed to the fact that we predict 13 masses with yellow symbols for which there are not yet values available from the lattice groups. Of particular interest are the mass predictions for the second ensemble of HSC as recalled in Table XIV. For this ensemble, we have been informed that the HSC is currently computing various scattering observables. We will return to this issue below.

The section is closed with a brief discussion of the quark masses. Given the different fit scenarios of Table XV, their values can be computed for any lattice ensemble for which the pion and kaon masses are measured on a specified lattice volume, where again, here, we ignore discretization effects. Within a chiral Lagrangian approach, only ratios of the quark masses can be determined. This is so because only products of $B_{0} m$ or $B_{0} m_{s}$ occur. In Fig. 9, such ratios are confronted with corresponding ratios from the various lattice groups. While our values are given by open symbols, the lattice results are given by closed symbols. We follow here our convention that the open symbols are always on top of the closed symbols. An amazingly consistent pattern occurs. We note that the determination of the quark-mass ratios depends on the action used and may be quite involved due to nontrivial renormalization effects. Most straightforward are the results from HPQCD and ETMC $[47,59,68]$, in which it is stated that the quark-mass ratio remains unrenormalized. The PACS and LHPC collaborations made significant efforts to control their nontrivial renormalization effects in the quark masses $[10,17]$. As shown in our figure, all quark-mass ratios appear consistent with a universal set of chiral low-energy parameters as given in Table XV. All four fit scenarios lead to almost indistinguishable results for the quark masses. The small spread in the low-energy constants is not significant.

\section{LOW-ENERGY CONSTANTS FROM QCD LATTICE DATA}

We report on our efforts to adjust the low-energy parameters to the $D$-meson masses as evaluated by the various lattice groups. Our first observation is that the available data set is not able to determine a unique parameter set without additional constraints. Therefore, it would be highly desirable to evaluate the $D$-meson masses with $J^{P}=0^{-}$and $J^{P}=1^{-}$quantum numbers on further QCD lattice ensembles with unphysical pion and kaon masses.

Typically, solutions can be found with similar quality in the lattice data reproduction but quite different values for the low-energy parameters. This problem is amplified by

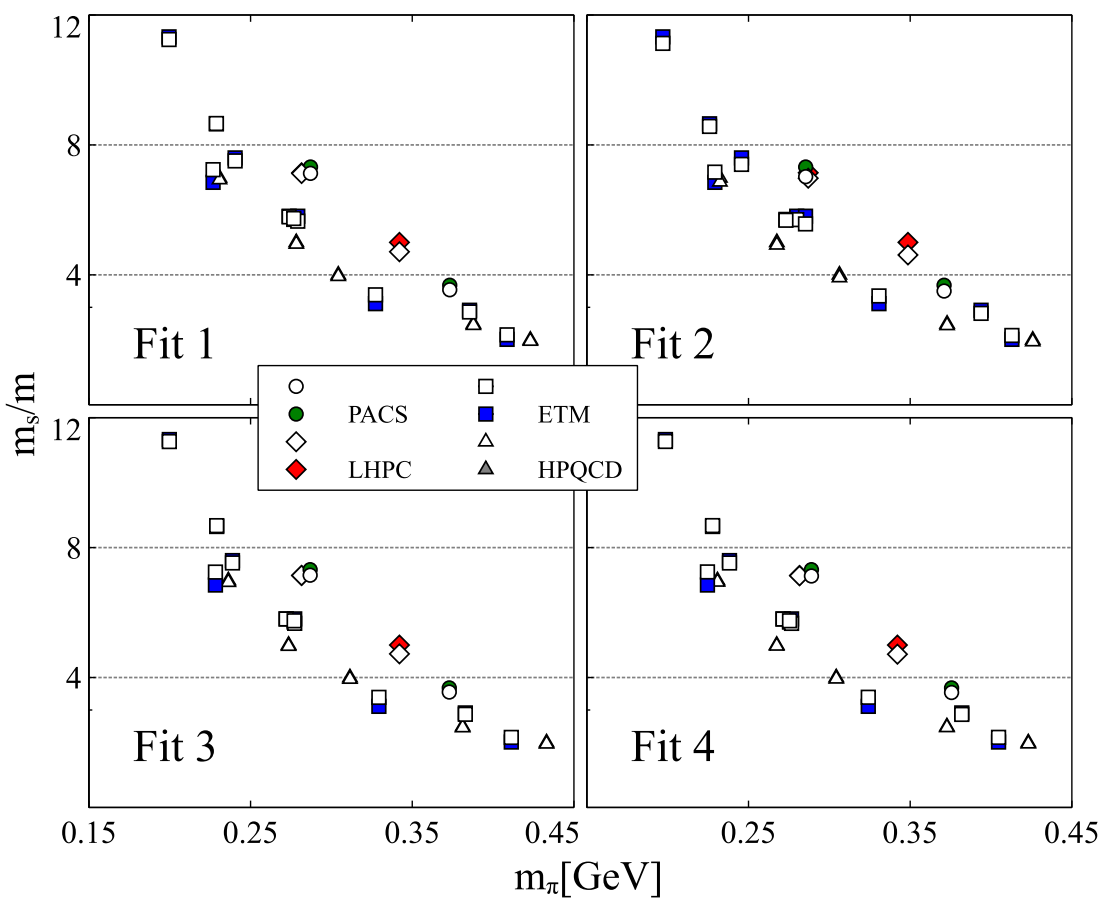

FIG. 9. The quark-mass ratios $m_{s} / m$ are shown for the various lattice ensembles considered. Closed symbols show the values from the lattice collaborations, and open symbols show our results. 
the unknown size of the underlying systematic error from discretization effects. Almost always, the size of the statistical errors given by the lattice groups is negligible, and it is expected that the systematic error is dominating the total error budget. In turn, it is unclear whether a parameter set with a better $\chi^{2}$ value is more realistic than a solution with a worse $\chi^{2}$. The $D$-meson masses may be overfitted.

To actually perform the fits is a computational challenge. For any set of the low-energy parameters, four coupled nonlinear equations are to be solved on each lattice ensemble considered. We apply the evolutionary algorithm of GENEVA 1.9.0-GSI [69] with runs of a population size 4000 on 100 parallel CPU cores.

In Table XVI, we collect four distinct fit scenarios that are constrained by additional input from first lattice results on some scattering observable. All four fit scenarios incorporate the s-wave scattering lengths of Ref. [10] into their $\chi^{2}$ functions. In addition, fits $2-4$ are adjusted to the scattering phases shifts of Ref. [23]. In fits 3 and 4, the subleading counterterms (49) are activated. All parameter sets reproduce the $D$-meson masses with a $\chi^{2} / N$ close to 1 , given an estimate for the systematic error in the range 5$10 \mathrm{MeV}$. In all fit scenarios, the four low-energy constants $c_{0,1}$ and $\tilde{c}_{0,1}$ are adjusted to recover the isospin-averaged physical $D$-meson masses with $J^{P}=0^{-}$and $J^{P}=1^{-}$ quantum numbers from the PDG [43]. This implies that deviations from leading-order large- $N_{c}$ or heavy-quark symmetry sum rules are considered for $c_{0,1}$ and $\tilde{c}_{0,1}$. In turn, we must not impose the heavy quark-symmetry relations $d_{n}=\tilde{d}_{n}$ for all $n=1, \ldots, 4$. Scale-invariant

TABLE XVI. The low-energy constants from a fit to the pseudoscalar and vector $D$-meson masses based on QCD lattice ensembles of the PACS-CS, MILC, ETMC, and HSC as described in the text. Each parameter set reproduces the isospin average of the empirical $D$-meson masses from the PDG.

\begin{tabular}{lrrrr}
\hline \hline & Fit 1 & Fit 2 & Fit 3 & Fit 4 \\
\hline$M(\mathrm{GeV})$ & 1.8762 & 1.9382 & 1.9089 & 1.8846 \\
$\Delta(\mathrm{GeV})$ & 0.1873 & 0.1876 & 0.1834 & 0.1882 \\
$c_{0}$ & 0.2270 & 0.3457 & 0.2957 & 0.3002 \\
$\tilde{c}_{0}$ & 0.2089 & 0.3080 & 0.2737 & 0.2790 \\
$c_{1}$ & 0.6703 & 0.9076 & 0.8765 & 0.8880 \\
$\tilde{c}_{1}$ & 0.6406 & 0.9473 & 0.8420 & 0.8583 \\
$c_{2}^{r}=\tilde{c}_{2}^{r}$ & -0.5625 & -2.1893 & -1.6224 & -1.3046 \\
$c_{3}^{r}=\tilde{c}_{3}^{r}$ & 1.1250 & 4.4956 & 3.2448 & 2.9394 \\
$c_{4}^{r}=\tilde{c}_{4}^{r}$ & 0.3644 & 2.0012 & 1.2436 & 0.9122 \\
$c_{5}^{r}=\tilde{c}_{5}^{r}$ & -0.7287 & -4.1445 & -2.4873 & -2.1393 \\
$d_{1}^{c}\left(\mathrm{GeV}^{-2}\right)$ & 1.8331 & 1.6937 & 1.6700 & 1.9425 \\
$\tilde{d}_{1}^{c}\left(\mathrm{GeV}^{-2}\right)$ & 1.6356 & 1.6586 & 1.4701 & 1.7426 \\
$d_{2}^{c}=\tilde{d}_{2}^{c}\left(\mathrm{GeV}^{-2}\right)$ & 1.0111 & 0.9954 & 0.8684 & 1.0032 \\
$d_{3}^{c}\left(\mathrm{GeV}^{-2}\right)$ & 0.1556 & 0.0679 & 0.1531 & 0.1109 \\
$\tilde{d}_{3}^{c}\left(\mathrm{GeV}^{-2}\right)$ & 0.2571 & 0.1640 & 0.2597 & 0.2143 \\
$d_{4}^{c}=\tilde{d}_{4}^{c}\left(\mathrm{GeV}^{-2}\right)$ & 0.8072 & 1.6392 & 0.8607 & 1.1255 \\
\hline \hline
\end{tabular}

expressions request $d_{1}^{c} \neq \tilde{d}_{1}^{c}$ and $d_{3}^{c} \neq \tilde{d}_{3}^{c}$ but permit the assumptions $d_{2}^{c}=\tilde{d}_{2}^{c}$ and $d_{4}^{c}=\tilde{d}_{4}^{c}$ [see (25)]. All four fit scenarios are based on the latter. In addition, we note that while fits 1 and 3 impose the leading-order large- $N_{c}$ relations

$c_{2}^{r}=-\frac{c_{3}^{r}}{2}, \quad c_{4}^{r}=-\frac{c_{5}^{r}}{2}, \quad \tilde{c}_{2}^{r}=-\frac{\tilde{c}_{3}^{r}}{2}, \quad \tilde{c}_{4}^{r}=-\frac{\tilde{c}_{5}^{r}}{2}$,

the remaining scenarios of fits 2 and 4 keep those parameters unrelated.

The quality with which the four scenarios reproduce the $D$-meson masses from the lattice ensembles is summarized in Table XVII. From the fact that all chi-square values are close to 1 for an ad hoc systematic error in between 5 and $10 \mathrm{MeV}$, we arrive at our estimate of an intrinsic systematic error of 5-10 MeV for the $D$-meson masses. All lowenergy parameters are in qualitative agreement with the first rough estimates in (7). On the other hand, we find significant tension with the low-energy parameters as obtained in Refs. [11,36,70,71]. The parameters of fit 2 are reasonably close to the two sets claimed in Ref. [10] with the notable exception of $c_{1}$, which differs by about a factor 2. Despite the considerable variations in the lowenergy constants, we deem all four parameter sets acceptable from the perspective of describing the $D$-meson

TABLE XVII. The table shows the impact of an ad hoc systematic error (that is added to the statistical error in mean quadrature) on the chi-square values of the various lattice data sets. The set of lattice data fitted is described in the text. The corresponding low-energy parameters of fits 1-4 are given in Table XVI.

\begin{tabular}{lccccc}
\hline \hline & Fit 1 & Fit 2 & Fit 3 & Fit 4 & $\begin{array}{c}\text { Systematic } \\
\text { error }(\mathrm{MeV})\end{array}$ \\
\hline$\chi_{\text {PACS-CS }}^{2} / N$ & 0.5054 & 0.8721 & 0.5329 & 0.4824 & 10 \\
& 1.6153 & 2.6456 & 1.9222 & 1.6726 & 5 \\
$\chi_{\text {LHPC }}^{2} / N$ & 0.0999 & 1.6006 & 0.3911 & 0.1574 & 10 \\
& 0.3659 & 5.9049 & 1.4524 & 0.5851 & 5 \\
$\chi_{\text {HPQCD }}^{2} / N$ & 0.9430 & 0.9131 & 1.2962 & 1.0606 & 10 \\
$\beta \simeq 6.76$ & 3.7132 & 3.5877 & 5.1052 & 4.1814 & 5 \\
$\chi_{\text {HPQCD }}^{2} / N$ & 0.2468 & 0.2688 & 0.3393 & 0.4172 & 10 \\
$\beta \simeq 7.09$ & 0.9798 & 1.0662 & 1.3459 & 1.6495 & 5 \\
$\chi_{\text {ETMC }}^{2} / N$ & 0.4584 & 1.2096 & 0.9919 & 0.8367 & 10 \\
$\beta=1.90$ & 1.1053 & 2.8710 & 2.5727 & 2.1517 & 5 \\
$\chi_{\text {ETMC }}^{2} / N$ & 0.6546 & 1.5087 & 1.0253 & 0.8279 & 10 \\
$\beta=1.95$ & 1.6217 & 3.6038 & 2.5556 & 2.0590 & 5 \\
$\chi_{\text {ETMC }}^{2} / N$ & 0.1860 & 0.4915 & 0.4431 & 0.3572 & 10 \\
$\beta=2.10$ & 0.4061 & 1.1424 & 0.9964 & 0.7943 & 5 \\
$\chi_{\text {HSC }}^{2} / N$ & 0.1425 & 0.1710 & 0.4735 & 0.2622 & 10 \\
& 0.3757 & 0.5893 & 1.8550 & 0.9965 & 5 \\
\hline \hline
\end{tabular}


TABLE XVIII. Chi-square values from fits 1-4 for the s-wave scattering length of Ref. [10]. The first two ensembles of Table XII with a kaon mass smaller than $600 \mathrm{MeV}$ are considered in the chi-square function. The corresponding low-energy parameters of fits 1-4 are given in Table XVI.

\begin{tabular}{lcccc}
\hline \hline & Fit 1 & Fit 2 & Fit 3 & Fit 4 \\
\hline$\chi_{\text {s-wave scattering lengths }}^{2} / N$ & 0.9184 & 1.3849 & 2.2596 & 2.0597 \\
\hline \hline
\end{tabular}

masses. We repeat that it is unclear whether fit 1 should be trusted more, only because it would be compatible with a discretization error slightly smaller than the one for fit 4 . After all, a $5 \mathrm{MeV}$ systematic error would be an astonishingly small value.

We take up the additional constraints considered. In Ref. [10], a set of s-wave pion and kaon scattering lengths was computed on four different lattice ensembles as recalled in Table XII. Since only for the first two ensembles the kaon mass is smaller than our cutoff choice of $600 \mathrm{MeV}$, we include into our $\chi^{2}$ function only the scattering lengths from the first two ensembles of that table. The scattering lengths are computed in the infinite volume limit based on the parameter sets collected in Table XVI.

We apply the coupled-channel framework pioneered in Refs. $[6,8,9]$, which is based on the flavor $\mathrm{SU}(3)$ chiral Lagrangian. It relies on the on-shell reduction scheme developed in Refs. [41,72], which can be justified if the interaction is of short-range nature or the long-range part is negligible $[73,74]$. Fortunately, this appears to be the case for the s-wave interactions of the Goldstone bosons off any of the $D$ mesons. In these and the current work, the coupled-channel interaction is approximated by tree-level expressions. Coupled-channel unitarity is implied by a particular summation scheme formulated in terms of scalar loop functions evaluated with physical meson masses and relativistic kinematics.

An alternative chain of works based on a somewhat different treatment of the coupled-channel effects is Refs. [10-13,36,71]. We did a careful comparison of the three available sources for the flavor structure of the coupled-channel interaction [9-11]. We find two discrepancies among the original works $[9,10]$ in which we do take into account the different phase conventions used in the two works for the isospin states. The two discrepancies are in the $(I, S)=(1 / 2,0)$ sector. One is traced as a misprint, in $C_{W T}$ of Table 2 of Ref. [9], in which the two entries 13 and 22 need to be interchanged (see Ref. [8]). The second one we attribute to a misprint in Ref. [10]. Unfortunately, we were not able to relate to the flavor coefficients shown in Ref. [11]. As compared to Refs. [9,10], there are more than ten unresolved contradictions.

In Table XVIII, we collect the $\chi^{2} / N$ values that characterize how well we reproduce the s-wave scattering length of Ref. [10] in our four fit scenarios. Note that we use here our estimates for the lattice scales $a_{\mathrm{LHPC}}$ as shown in Table XV. The table is complemented by Fig. 10,
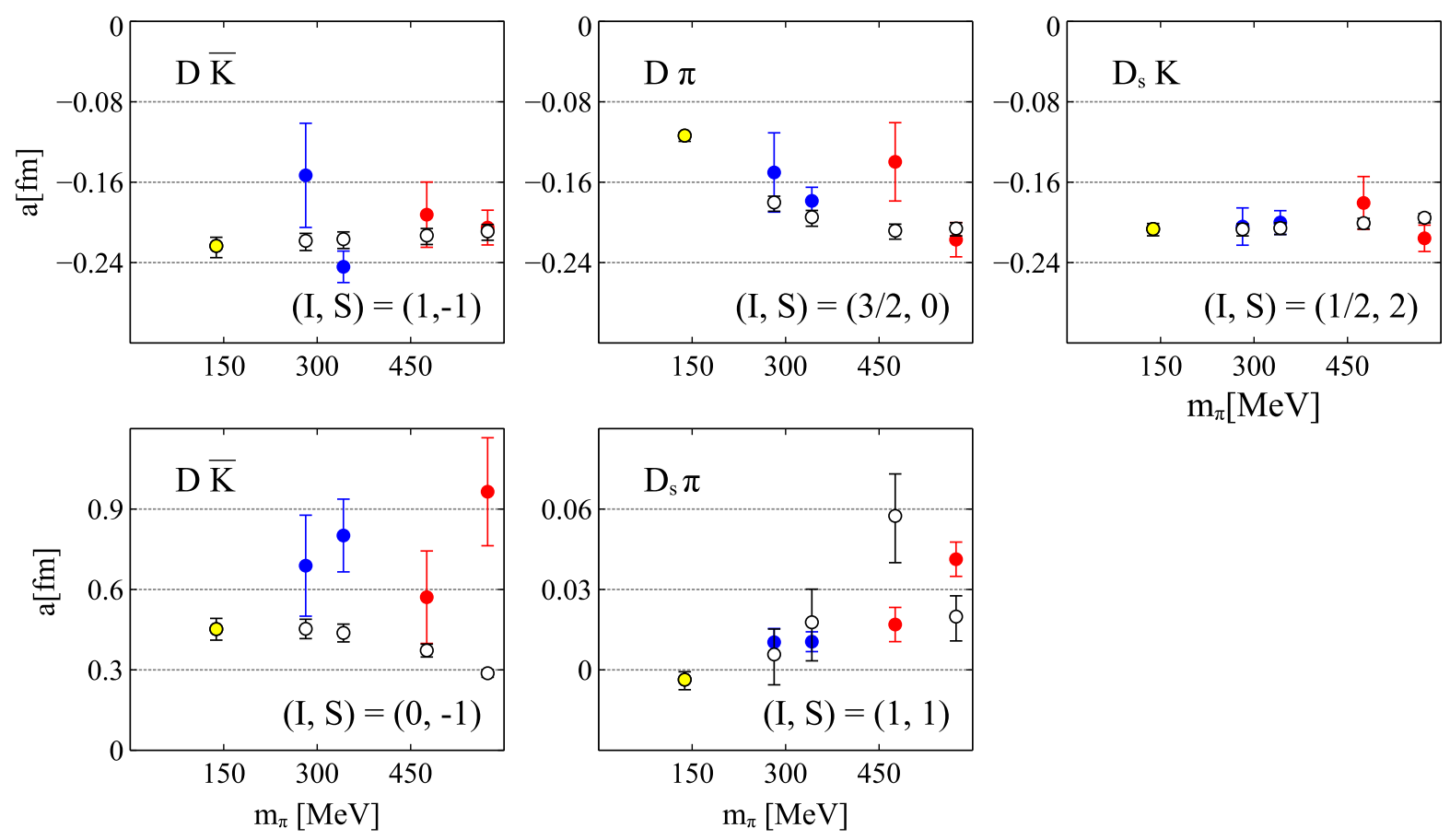

FIG. 10. s-wave scattering length from fit 4 as compared to predictions from Ref. [10]. The blue (red) data points show the scattering lengths for the ensembles in which the kaon mass is smaller (larger) than $600 \mathrm{MeV}$. The yellow points provide the physical value for the scattering lengths. 
in which a direct comparison of our results with the lattice data is provided for fit 4 . In the figure, the lattice data points, shown by filled symbols, are confronted with open symbols that represent our results. The error bars in the latter points reflect an estimate of the systematic uncertainty in our computation of the scattering lengths, in which we should state that the $\chi^{2}$ values in Table XVIII are computed always in terms of the center value of our prediction. Our systematic error estimate is implied by a variation of the matching scale $\mu_{M}$ around its natural value $[6,8,9]$. The error bars are implied by $\Delta \mu_{M}= \pm 100 \mathrm{MeV}$ with $\mu_{M} \rightarrow \mu_{M}+\Delta \mu_{M}$. For a detailed discussion of why $\Delta \mu_{M}$ cannot be chosen much larger without jeopardizing the approximate implementation of crossing symmetry, we refer to the original works [41,72]. It is important to recall that dialing the matching scale slightly off its natural value does not affect our self-consistent determination of the $D$-meson masses. The latter is a convenient tool to estimate the uncertainties of the unitarization process.

In the upper panels of Fig. 10, we show the channels that are dominated by a repulsive Tomozawa-Weinberg interaction term [8]. In terms of a flavor SU(3) multiplet classification, they belong to a flavor 15-plet that cannot be reached within the traditional quark-model picture. A minimal four-quark state configuration is required. In contrast, in the lower panels, channels that belong to the exotic flavor sextet sector, in which the leading TomozawaWeinberg interaction shows a weak attraction, are presented [8]. As pointed out in Refs. [3,6,8,9], depending on the size of chiral correction terms, exotic resonance states may be formed by the chiral dynamics. Final-state interactions distort the driving leading-order term and ultimately generate the more complicated quark-mass dependence as seen in the figure. We discriminate results based on ensembles with a kaon mass larger or smaller than $600 \mathrm{MeV}$ by distinct colored symbols. With red symbols, we indicate that the kaon mass is larger than our cutoff value, and therefore chiral dynamics is not expected to be reliable. A fair reproduction of all relevant scattering lengths is seen in Fig. 10. Our predictions for the scattering lengths at the physical point are also included by the additional yellow filled points farthest to the left.

We conclude that with the constraints set by scattering lengths of Ref. [10] we cannot rule out any of our four fit scenarios in Table XVI.

\section{SCATTERING PHASE SHIFTS FROM QCD LATTICE DATA}

In this section, we finally present an additional constraint on the low-energy parameters that provide a clear criterion that of the four fit scenarios is most reliable and should be used in applications. Recently, HSC computed $\pi D$ phase shifts in both isospin channels. The results are based on the ensemble recalled in Table XIV. Given our four parameter sets, we can compute those observable at the given unphysical pion and kaon masses. We do this for all four parameter sets.

It is necessary to explain how we compare with those lattice results. Ultimately, one should compute the various discrete levels the collaboration computed and then apply the Lüscher method $[75,76]$ to extract the coupled-channel scattering amplitudes. This requires an ansatz for the form of the reaction amplitudes. In the case of a single-channel problem, this can be analyzed in a model-independent manner. In turn, for $\pi D$ scattering in the $I=3 / 2$ channel, we can compare our results with the single-energy phase shifts as taken from Fig. 20 of Ref. [23] at different center-of-momentum energies $E=\sqrt{s}-m_{\pi}-M_{D}$. They are to be confronted with the four lines from our four fit scenarios. In the figure of Table XIX, we see that the two red lines are significantly off the lattice data points, where with those lines fit 1 and 2 are presented. This is the case even though in fit 2 an attempt was made to reproduce the $\pi D$ phase shifts from Ref. [23]. Note that in fit 1 we ignored any of the latter. We assure that our conclusions are stable against a reasonable variation of the matching scale in this sector.

Based on this observation, we made our ansatz for the scattering amplitudes more quantitative by the consideration of an additional set of low-energy constants relevant at chiral order 3. Such terms were constructed in Refs. $[77,78]$ to take the form

$$
\begin{aligned}
\mathcal{L}_{3}= & 4 g_{1} D\left[\chi_{-}, U_{\nu}\right]_{-} \hat{\partial}^{\nu} \bar{D} \\
& -4 g_{2} D\left(\left[U_{\mu},\left[\hat{\partial}_{\nu}, U^{\mu}\right]_{-}\right]_{-}+\left[U_{\mu},\left[\hat{\partial}^{\mu}, U_{\nu}\right]_{-}\right]_{-}\right) \hat{\partial}^{\nu} \bar{D} \\
& -4 g_{3} D\left[U_{\mu},\left[\hat{\partial}_{\nu}, U_{\rho}\right]_{-}\right]_{-}\left[\hat{\partial}^{\mu},\left[\hat{\partial}^{\nu}, \hat{\partial}^{\rho}\right]_{+}\right]_{+} \bar{D}+\text { H.c. }
\end{aligned}
$$

Our motivation to consider such terms is slightly distinct from the one followed in Refs. [77,78]. From the previous work [6], we expect the light vector meson degrees of freedom (d.o.f.) to play a crucial role for the considered physics. Ultimately, we would like to consider them as active d.o.f. This is beyond the scope of the current work. Here, we consider the low-energy constants as a phenomenological tool to more accurately integrate out the light 
TABLE XIX. While the solid lines are from fits 2 and 4 , the dashed lines are with respect to fits 1 and 3. The lattice data are from Ref. [23].

\begin{tabular}{lcccc}
\hline \hline & Fit 1 & Fit 2 & Fit 3 & Fit 4 \\
\hline$g_{1}\left(\mathrm{GeV}^{-1}\right)$ & 0 & 0 & 0.2240 & 0.2338 \\
$g_{2}\left(\mathrm{GeV}^{-2}\right)$ & 0 & 0 & 0.5405 & 0.4663 \\
$g_{3}\left(\mathrm{GeV}^{-4}\right)$ & 0 & 0 & 0.0399 & 0.0299 \\
\hline \hline
\end{tabular}

vector meson d.o.f. In the scenarios of fits 3 and 4, the contributions of the $g_{n}$ are worked into the coupled-channel interaction. Their values are displayed in Table XIX, which consecutively lead to a significantly improved reproduction of the scattering phase shift.

We proceed with the coupled-channel $\pi D$ system with $I=1 / 2$ for which its determination of the three phase shifts and inelasticities is more involved. Some model dependence may enter the analysis. In Ref. [23], an estimate of the latter was accessed by allowing a quite large set of different forms of the ansatz for the coupledchannel amplitudes. That then led to two error bands in their plotted phase shifts and inelasticity parameters. The smaller one shows the statistical uncertainty, and the larger

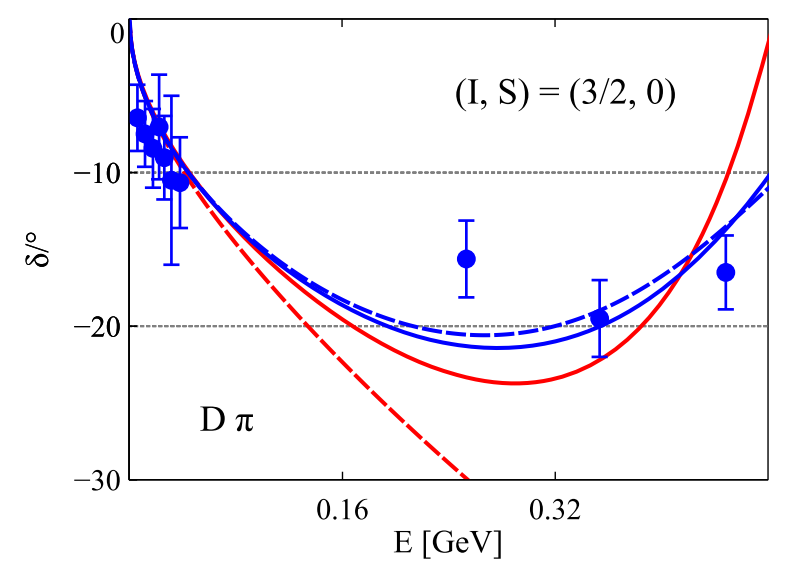

one includes also the systematic error. In Figs. 9 and 10 of Ref. [23], it is shown, in addition, on how many levels their results are based on in a given energy bin. Above the $\pi D$ and below the $\bar{K} D_{s}$ thresholds, there are three clusters of levels. We take their center and translate those into singleenergy phase shifts and inelasticities with error bars taken from the estimated uncertainties. In Fig. 11, those "lattice data" points are shown and confronted with our results from the four fit scenarios. In addition, a fourth lattice data point at energies above the $\bar{K} D_{s}$ threshold is also included in the figure but shown in red symbols. We do have some reservation toward those points, since the number of closeby energy levels is quite scarce. This is particularly troublesome since here it is a true three-channel system
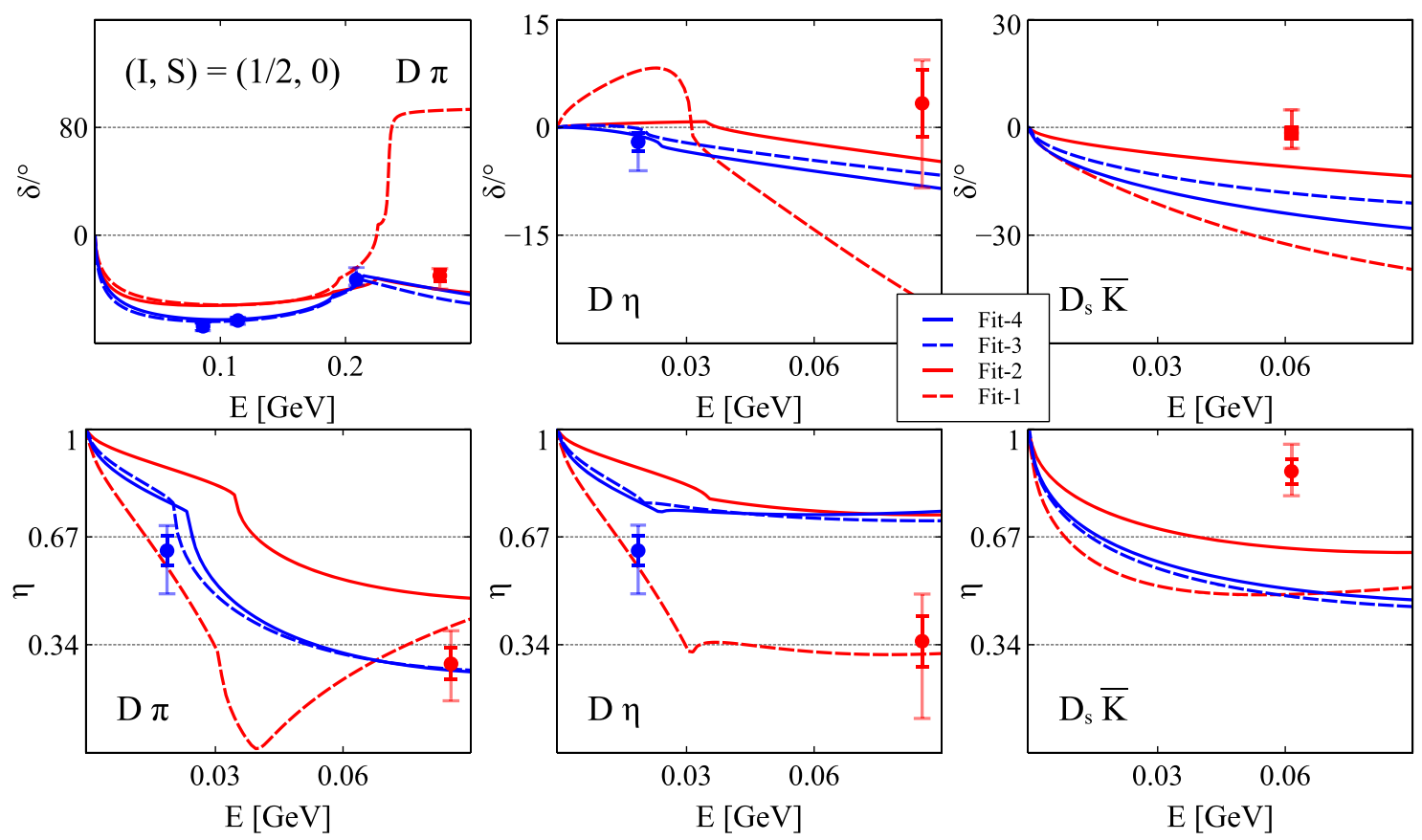

FIG. 11. Phase shifts with $(I, S)=(1 / 2,0)$ from fits $1-4$ as compared to lattice data from Ref. [23]. While the solid lines are from fits 2 and 4, the dashed lines are with respect to fits 1 and 3 . The two red lines present the disfavored scenarios from fits 1 and 2 . We apply the somewhat unusal convention of the lattice group in which the phase shift at threshold is normalized to zero even in the presence of a bound state. 
that would need more rather than a fewer number of levels to unambiguously determine the scattering amplitude. In turn, the particular choice of ansatz is expected to play a much more significant role in the determination of the red lattice data points. We conclude that the error bars must be significantly underestimated for those points.

Figure 11 confirms our conclusions from the previous Table XIX that only fits 3 and 4 may be expected to be faithful. The $\pi D$ and $\eta D$ phase shift points are highly discriminative among the four fit scenarios. Fits 3 and 4 describe the lattice data in Fig. 11 significantly better than fits 1 and 2. Since fit 4 is doing better in the $D$-meson masses, also in the s-wave scattering lengths, one may identify fit 4 to be the most promising candidate for making reliable predictions.

There is a further piece of information provided by HSC in the given ensemble. The mass $M_{B}$ of a bound state just below the $\pi D$ threshold is predicted. It is a member of the conventional flavor antitriplet, the formation of which was predicted by chiral dynamics unambiguously $[8,9]$. Within the given error, it is not distinguishable from the $\pi D$ threshold value. The following bound is derived from data published by HSC,

$$
\epsilon_{B}=\frac{m_{\pi}+M_{D}}{M_{B}}-1<0.001
$$

at the one sigma level. We compute this value in the four fit scenarios with

$$
10^{3} \epsilon_{B}=\left\{\begin{array}{cccc}
\text { Fit } 1 & \text { Fit } 2 & \text { Fit } 3 & \text { Fit } 4 \\
8.0 & 5.4 & 4.3 & 5.7
\end{array}\right.
$$

where we find discrepancies for the bound-state mass of the order of our resolution of 5-10 MeV. For a consistency check, we exploit the uncertainties in the unitarization process, by tuning the matching scale to meet the condition (50) for fits 1-4. This is achieved, for instance, with $\Delta \mu_{M} \simeq$ $69 \mathrm{MeV}$ and $\Delta \mu_{M} \simeq 86 \mathrm{MeV}$ in fits 3 and 4, respectively, where we emphasize that with $\Delta \mu_{M}$ the determination of the $D$-meson masses is not affected. Then, we reconsider the phase shifts and inelasticities and find that altogether the impact of such a change of the matching scale is quite moderate. While now fit 1 goes almost perfectly through the three blue lattice data points for the $\pi D$ phase shift, the lines of fits 3 and 4 are slightly below those points. The crucial observation is that the significant disagreement with the single blue $\eta D$ phase shift is persistent in the fit 1 scenario, and therefore fit 4 must remain our favorite choice.

We wish to make one comment on fit 1 since it is particularly interesting despite its deficiencies: a clear signal of a member of the exotic sextet state is visible in the $\pi D$ phase shift. It shows a significant variation a little right of the last blue lattice point. We deem it unfortunate that exactly in this region there are not yet sufficient consolidated lattice points that may rule out our first fit scenario unambiguously available. Note, furthermore, that our fit 1 scenario, which did not take any of the scattering observables from HSC into account, is disfavored mainly by one feature of the HSC results in the $(I, S)=(1 / 2,0)$ sector. The single blue value for $\eta D$ phase shift is significantly off the line of Fit 1 . It would be interesting to make the ansatz used by HSC for the coupled-channel amplitude more flexible and allow for an exotic-state coupling dominantly to the $\eta D$ channel. One
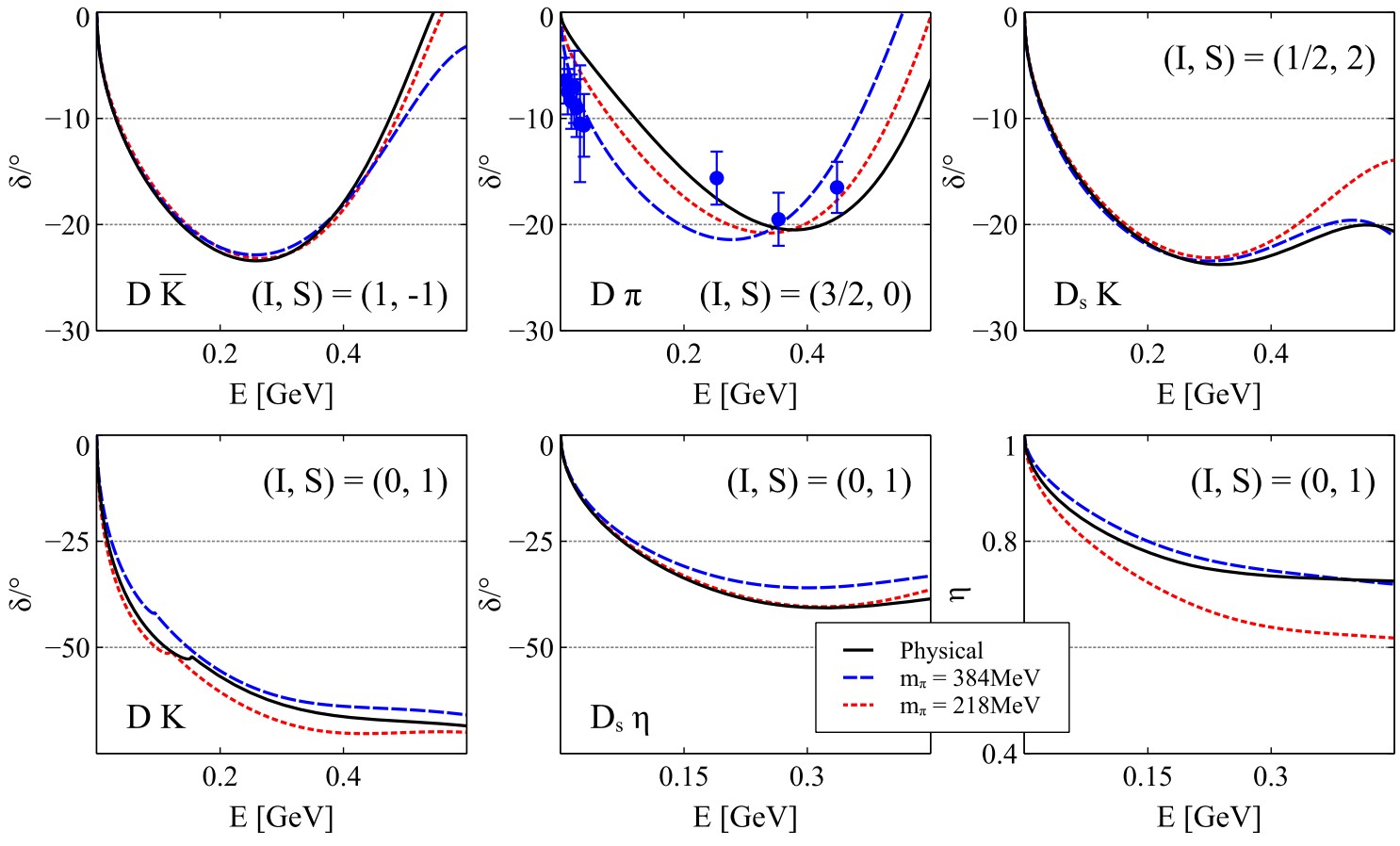

FIG. 12. Predictions for phase shifts from fit 4 for the physical point but also for pion and kaon masses as shown in Table XIV. 
may speculate that this exercise could show that the claimed uncertainty for this lattice point is underestimated significantly. If this happens, our fit 1 scenario may come into the game again. This may be so even though HSC appears to reject our fit 1 scenario based on their results in the $(I, S)=$ $(3 / 2,0)$ sector. Here, the reader should be cautioned that we cannot fully rule out that the phenomenological treatment of the third-order effects is fooling us. More detailed studies are required to substantiate our conclusions.

In the following, we take our best-fit scenario of fit 4 and provide a thorough documentation of its consequences. In Figs. 12 and 13, all phase shift and inelasticity parameters are shown for all possible combinations of $(I, S)$. In Fig. 12, we present the channels in which no exotic signals
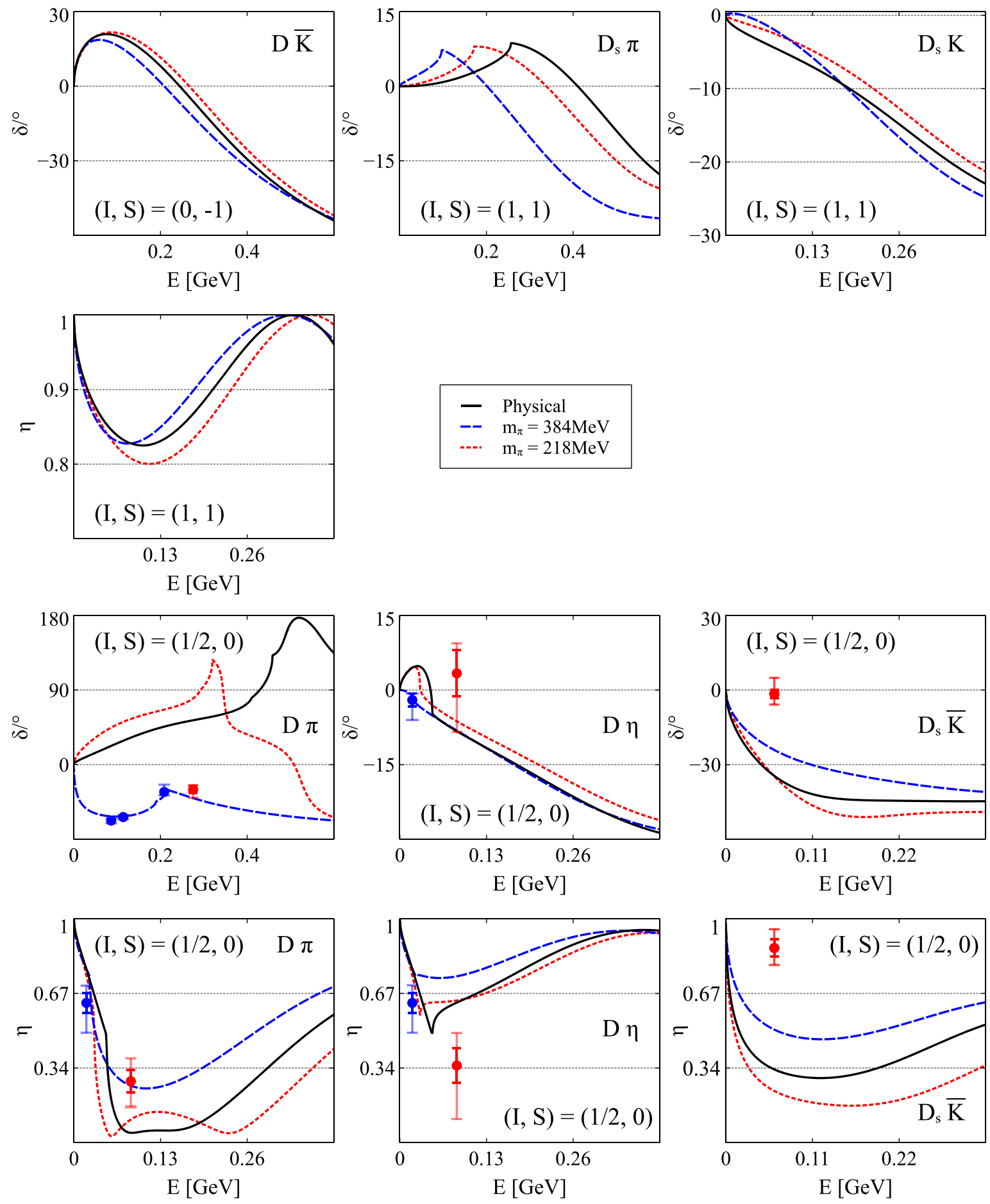

FIG. 13. Predictions for phase shifts from fit 4 for the physical point but also for pion and kaon masses as shown in Table XIV. 
are expected. Indeed, the evolution from the two HSC ensembles of Table XIV with unphysical quark masses to the physical point is smooth and unspectacular. While the solid black lines correspond to the physical point, the dashed and dotted lines correspond to the two HSC cases, in which the dashed lines are with respect to the upper ensemble of Table XIV. We refrain from including our estimate of the systematic uncertainty from a variation of the matching scale, because, first of all, it is a small effect and, second, it obscures the clarity of the figures.

We advance to the exotic sectors with $(I, S)=(0,-1)$ and $(1,1)$. With the upper two panels of Fig. 13, we demonstrate that, here, the evolution from the two HSC ensembles to the physical point is still smooth but quantitatively more significant, particularly in the two-channel system with $(I, S)=(1,1)$. The corresponding amplitudes are characterized by strong cusp effects at threshold. The latter reflect some weak attraction present in those channels being members of the flavor sextet.

Most striking are our predictions for the quark-mass dependence of the $(I, S)=(1 / 2,0)$ sector, which we present with the lower two panels of Fig. 13. The line conventions are identical to the ones used in the previous figures. The largest effect is seen in the $\pi D$ phase shift. Going from the HSC ensembles to the physical point, it even changes sign. Here, we see a clear signal for a member of the exotic flavor sextet state. The $\pi D$ phase shift passes through $90^{\circ}$ in between the $\eta D$ and $\bar{K} D_{s}$ thresholds. We checked that the amplitudes $\eta D \rightarrow \eta D$ and also $\bar{K} D_{s} \rightarrow \bar{K} D_{s}$ show a well-defined resonance structure, with a width significantly smaller than the $300-400 \mathrm{MeV}$ of the flavor antitriplet partner at lower masses. We find this to be a spectacular confirmation of the leading-order prediction of this state advocated since 15 years ago by one of the authors (see Ref. [3]). It is amusing to see that the clear signature of this state at the physical point may not be seen at the studied HSC ensemble with unphysically large pion masses. Most exciting is the most recent claim in Ref. [79] that this state can be seen in data from LHCb $[80,81]$.

\section{ISOSPIN-VIOLATING DECAY OF $D_{s 0}^{*}(2317)$ FROM QCD LATTICE DATA}

A most striking prediction of chiral dynamics is the formation of the $D_{s 0}^{*}(2317)$ as a coupled-channel hadronic molecule with significant components in the $K D$ and $\eta D_{s}$ two-body states [8]. At leading order in a chiral expansion, the coupled-channel interaction is predicted by the Tomozwa-Weinberg term that is parametrized only by the pion-decay or kaon-decay constants, $f_{\pi}$ or $f_{K}$, driven into their chiral flavor $S U(3)$ limit with $f_{\pi, K} \rightarrow f$.

This term dominates the s-wave coupled-channel force of the Goldstone bosons with the pseudoscalar and vector
$D$ mesons. The force is short ranged; it may be visualized in terms of a vector meson $t$-channel exchange process with properly adjusted coupling constants. In contrast to a widespread confusion in the field, there are hadronic molecular states that are not driven by a long-range force as provided by an exchange process involving the pion. The challenge is to control and predict such short-range forces.

The original work [8] was taken up by many authors $[6,9,11-14,71,79,82,83]$ who confirm this universal picture. The challenge is to make this approach more quantitative by controlling chiral correction terms. A first attempt was made in Refs. [6,9] based on rough assumptions on the $\pi D$ invariant mass distributions. A more sophisticated approach was pursued in Refs. [10,12], in which first QCD lattice data on some s-wave scattering lengths were used. With the significantly improved and extended lattice data set, the determination of the low-energy constants, as achieved in our work, is expected to be more controlled and reliable.

In this section, we focus on a particular property of the $D_{s 0}^{*}(2317)$, its isospin-violating hadronic decay width. Since its mass is below the $K D$ threshold and it carries isospin 0 , it can decay into the $\pi D$ channel only via isospin-violating processes. Estimates of that width within typical quark-model approaches predict such a width of less than $10 \mathrm{keV}$ [84]. This is contrasted by estimates from chiral-coupled-channel approaches. Here, already, the leading-order Tomozawa-Weinberg predicts a width of about $75 \mathrm{keV}$ as demonstrated first in Ref. [6]. A corresponding computation with similar physics input but less stringent framework arrived at a similar value [82]. This is to be compared to the significantly larger values of about $140 \mathrm{keV}$ in Ref. [6] and later with even an error estimate of $(133 \pm 22) \mathrm{keV}$ [10]. The latter two works implemented chiral correction terms, in which the more sophisticated approach [10] was based on additional constraints from some early lattice data.

The results of our study for the decay width are collected in Table XX for all four fit scenarios. They are based on the framework as detailed in Ref. [6]. Since the mass of the $D_{s 0}^{*}(2317)$ was not tuned in any of our fits, we again use the uncertainty in the unitarization and adjust the matching scale to recover the precise mass of the $D_{s 0}^{*}(2317)$. This is achieved with $50 \mathrm{MeV}<\Delta \mu_{M}<100 \mathrm{MeV}$ in the four scenarios. Besides the low-energy constants determined in our work, the computation of the width parameter depends crucially on the mixing angle $\epsilon$ of the $\pi_{0}-\eta$ system.

TABLE XX. Prediction for the isospin-violating decay width of the $D_{s 0}^{*}(2317)$ in the four fit scenarios of Table XVI.

\begin{tabular}{lcrrrr}
\hline \hline & Fit 1 & Fit 2 & Fit 3 & Fit 4 & $\epsilon$ \\
\hline$\Gamma_{D_{s 0}^{*}(2317) \rightarrow \pi_{0} D_{s}}(\mathrm{keV})$ & 61.1 & 54.1 & 88.6 & 80.1 & 0.0100 \\
& 74.6 & 68.4 & 115.8 & 104.4 & 0.0122 \\
\hline \hline
\end{tabular}


According to Ref. [67], it is determined by the quark masses as follows:

$$
\frac{\sin (2 \epsilon)}{\cos (2 \epsilon)}=\sqrt{3} \frac{m_{d}-m_{u}}{2 m_{s}-m_{u}-m_{d}} .
$$

While in Ref. [6] the value $\epsilon=0.010$ (1) was taken from Ref. [67], an updated estimate $\epsilon=0.0129(7)$ was used in Ref. [10]. Here, we consider the impact of a recent and more precise lattice determination of the quark masses by ETMC [59]. This leads to a significantly lower estimate $\epsilon=0.0122$ (18) on which our faithful results in Table XX are based.

Since we argued that the lattice data of HSC rule out fits 1 and 2, we estimate the isospin-violating hadronic width of the $D_{s 0}^{*}(2317)$ with (104-116) keV, somewhat lower than the previous claimed value of $(133 \pm 22) \mathrm{keV}$ [10].

\section{SUMMARY AND CONCLUSIONS}

We studied the chiral extrapolation of charmed-meson masses based on the three-flavor chiral Lagrangian formulated with pseudoscalar and vector charmed fields. Here, the recent approach by the authors constructed for the chiral extrapolation of the baryon ground-state masses was adapted to the charm sector successfully, and good convergence properties for the chiral extrapolation were observed. Within the framework, the chiral expansion was formulated in terms of physical masses. While an attempt was made to remove all model dependence, a residual scheme dependence cannot be ruled out at this stage. All $D$-meson masses arise in a manifest scaleinvariant manner. The framework was applied to lattice data such that an almost unique set of low-energy constants was established. While we considered finite volume effects systematically, we did not implement discretization effects. In turn, a fully systematic error analysis was outside the realm of our present study.

The low-energy parameters were adjusted to QCD lattice data at $\mathrm{N}^{3} \mathrm{LO}$, where large- $N_{c}$ sum rules or relations that followed in the heavy charm quark-mass limit were used systematically. We considered lattice data based on ensembles of PACS-CS, MILC, ETMC, and HSC with pion and kaon masses smaller than $600 \mathrm{MeV}$. Besides taking into account constraints from the $D$-meson masses from the various lattice groups, we also considered first results on scattering observables in particular from HSC. Only with the latter, in particular, HSC's estimate of the $\eta D$ phase shift, we arrived at a rather well-defined parameter set, in terms of which we made predictions. The data set on the $D$ meson masses together with constraints from s-wave scattering lengths is not sufficient to nail down the set of low-energy constants.

We computed 15 phase shifts and inelasticities at physical quark masses and also for an additional HSC ensemble. Such results can be scrutinized by lattice QCD with available computing resources and technology. In addition, we predict the isospin-violating strong decay width of the $D_{s 0}^{*}(2317)$ to be (104-116) keV. Given our favorite set of low-energy parameters, we find a clear signal for a member of the exotic flavor sextet states in the $\eta D$ channel, below the $\bar{K} D_{s}$ threshold.

To further substantiate the claimed chiral low-energy parameters, it is necessary to take additional data on QCD lattices, in particular, at unphysical quark masses. Our predictions are relevant for the PANDA experiment at FAIR, in which the width of the $D_{s 0}^{*}(2317)$ may be accessible by a scan experiment [85]. Also, the invariant $\eta D$ mass distribution, in which we expect a signal from an exotic flavor sextet state, may be accessed by the efficient detection of neutral particles with the available calorimeter.

\section{ACKNOWLEDGMENTS}

M. F. M. L. thanks Kilian Schwarz and Jan Knedlik for significant support on distributed computing issues. Particular thanks go to Walter Schön, who is operating the high performance computing (HPC) cluster at GSI with his department in an outstanding manner. We are grateful to Rüdiger Berlich of Gemfony scientific UG for help with their optimization library Geneva. Marc Wagner and Sinead Ryan are acknowledged for stimulating discussions. We thank Feng-Kun Guo for help in correctly using Ref. [59].

\section{APPENDIX A}

In this appendix, we collect all dimensionless coefficients that are needed in the various power-counting decompositions of the renormalized loop function (14). Here, we focus on the pseudoscalar $D$ mesons for which we find

$$
\begin{aligned}
& \alpha_{1}=\frac{(2 M+\Delta)^{2}}{4 M^{2}}, \quad \alpha_{2}=\frac{2 M^{2}+2 \Delta M+\Delta^{2}}{2 M^{2}}, \quad \alpha_{3}=1, \\
& \gamma_{1}=\frac{2 M+\Delta}{M} \log \frac{\Delta(2 M+\Delta)}{(M+\Delta)^{2}}, \quad \gamma_{2}=-\frac{2 M^{2}+2 \Delta M+\Delta^{2}}{M(2 M+\Delta)} \log \frac{\Delta(2 M+\Delta)}{(M+\Delta)^{2}}-\frac{M}{2 M+\Delta}, \\
& \gamma_{3}=\frac{M}{2 M+\Delta}, \quad \gamma_{4}=-2 \frac{M(M+\Delta)^{2}}{(2 M+\Delta)^{3}} \log \frac{\Delta(2 M+\Delta)}{(M+\Delta)^{2}}+\frac{M^{3}}{2(2 M+\Delta)^{3}}, \quad \gamma_{5}=\frac{M(M+\Delta)^{2}}{(2 M+\Delta)^{3}},
\end{aligned}
$$




$$
\begin{aligned}
& \delta_{1}=\gamma_{1}-\frac{2 M+\Delta}{M} \log \frac{2 \Delta}{(M+\Delta)}, \quad \delta_{2}=\gamma_{2}+\frac{2 M^{2}+2 \Delta M+\Delta^{2}}{M(2 M+\Delta)} \log \frac{2 \Delta}{M}+\frac{2 M+\Delta}{4 M}+2\left(\delta_{3}-\gamma_{3}\right) \log \frac{M+\Delta}{M}, \\
& \delta_{3}=\gamma_{3}-\frac{2 M^{2}+2 \Delta M+\Delta^{2}}{2 M(2 M+\Delta)}, \quad \delta_{5}=0, \\
& \delta_{4}=\gamma_{4}+\frac{2 M(M+\Delta)^{2}}{(2 M+\Delta)^{3}} \log \frac{2 \Delta}{M}-\frac{4 M^{2}+\Delta(4 M+5 \Delta)}{32 M(2 M+\Delta)}+2\left(\delta_{5}-\gamma_{5}\right) \log \frac{M+\Delta}{M}, \\
& \delta_{6}=\frac{2 M+\Delta}{2 M} \frac{\partial}{\partial \Delta} \frac{2 M \Delta}{2 M+\Delta}\left(\gamma_{1}-\delta_{1}\right)+\delta_{1}, \quad \delta_{7}=\gamma_{2}+\frac{1}{2}\left(\gamma_{1}-\delta_{1}\right) \frac{\Delta^{2}}{(2 M+\Delta)^{2}}, \\
& \beta_{1}=\Delta \frac{\partial}{\partial \Delta} \alpha_{1} \frac{2 M+\Delta}{2 M}, \quad \beta_{2}=\Delta^{2} \frac{\partial}{\partial \Delta} \frac{\alpha_{1} \delta_{2}}{\Delta}, \quad \beta_{3}=\Delta^{2} \frac{\partial}{\partial \Delta} \frac{\alpha_{1} \delta_{3}}{\Delta}, \quad \beta_{4}=\Delta \gamma_{1} \frac{\partial}{\partial \Delta} \alpha_{1}, \quad \beta_{5}=\Delta \frac{\partial}{\partial \Delta} \alpha_{1} \delta_{1}, \\
& \beta_{6}=\frac{\Delta^{2} \partial^{2}}{\partial \Delta \partial \Delta}\left(\alpha_{1} \frac{2 M+\Delta}{2 M}\right), \quad \beta_{7}=\Delta \frac{\Delta^{2} \partial^{2}}{\partial \Delta \partial \Delta} \frac{\alpha_{1} \delta_{2}}{\Delta} \quad \beta_{8}=\Delta \frac{\Delta^{2} \partial^{2}}{\partial \Delta \partial \Delta} \frac{\alpha_{1} \delta_{3}}{\Delta}, \quad \beta_{9}=\gamma_{1} \frac{\Delta^{2} \partial^{2}}{\partial \Delta \partial \Delta} \alpha_{1}, \\
& \beta_{10}=\frac{\Delta^{2} \partial^{2}}{\partial \Delta \partial \Delta} \alpha_{1} \delta_{1}, \quad \beta_{11}=-\frac{1}{4} \alpha_{1} \frac{M}{2 M+\Delta}+\left(\alpha_{1}-\alpha_{2}\right) \frac{(2 M+\Delta) M}{2 \Delta^{2}} .
\end{aligned}
$$

While the $\alpha_{i}$ characterize the chiral expansion of the coefficients in front of $\bar{I}_{Q R}$ and $\bar{I}_{Q}$ in (16), the $\gamma_{i}$ and $\delta_{i}$ follow from a chiral expansion of $\bar{I}_{Q R}$ with $M_{H}=M$ and $M_{R}=M+\Delta$ and $m_{Q}<\Delta$. The coefficients $\beta_{i}$ are required in (44), (45), (A6).

We turn to the chiral domain (34), in which the bubble-loop contributions to the $D$-meson masses generate a renormalization of the low-energy parameters $d_{i}$. Such terms are proportional to the product of two quark masses (9). We provide detailed results with

$$
\begin{aligned}
\Pi_{H}^{(4-\chi)} & \rightarrow \sum_{Q \in[8]} \sum_{R \in\left[1^{-}\right]}\left(\frac{G_{Q R}^{H}}{8 \pi f}\right)^{2}\left\{\gamma_{d}^{(1)} m_{Q}^{2} \Pi_{R}^{(2)}+\gamma_{d}^{(2)} m_{Q}^{2} \Pi_{H}^{(2)}+\gamma_{d}^{(3)} \Pi_{R}^{(2)} \Pi_{R}^{(2)}+\gamma_{d}^{(4)} \Pi_{H}^{(2)} \Pi_{H}^{(2)}+\gamma_{d}^{(5)} \Pi_{R}^{(2)} \Pi_{H}^{(2)}\right\}, \\
d_{i} & \rightarrow \frac{1}{4} g_{P}^{2} \sum_{k=1}^{5} \frac{\Gamma_{d_{i}}^{(k)}}{(4 \pi f)^{2}} \gamma_{d}^{(k)},
\end{aligned}
$$

and

$$
\begin{aligned}
\gamma_{d}^{(1)}= & \frac{M}{2(M+\Delta)}\left[\frac{\partial}{\partial \Delta}\left(\alpha_{2} \Delta \gamma_{1}-\alpha_{1} \Delta \gamma_{2}\right)-\Delta \gamma_{1} \frac{\partial \alpha_{2}}{\partial \Delta}\right], \\
\gamma_{d}^{(2)}= & \frac{\Delta}{2 M}\left[\frac{\partial}{\partial M}\left(\alpha_{2} M \gamma_{1}-\alpha_{1} M \gamma_{2}\right)-\frac{1}{M} \gamma_{1} \frac{\partial}{\partial M}\left(\alpha_{2} M^{2}\right)\right]-\frac{M+\Delta}{M} \gamma_{d}^{(1)}, \\
\gamma_{d}^{(3)}= & -\frac{M}{4(M+\Delta)^{2}}\left(\frac{\partial \alpha_{1} \Delta^{2}}{\partial \Delta}\right)\left(\frac{\partial \gamma_{1} \Delta}{\partial \Delta}\right)-\frac{\alpha_{1} \Delta^{2} M}{4(M+\Delta)} \frac{\partial}{\partial \Delta}\left[\frac{1}{2(M+\Delta)}\left(\frac{\partial \gamma_{1} \Delta}{\partial \Delta}\right)\right], \\
\gamma_{d}^{(4)}= & -\frac{1}{8 M^{2}}\left(\frac{\partial}{\partial M}-\frac{\partial}{\partial \Delta}\right)^{2}\left(\alpha_{1} M \Delta^{3} \gamma_{1}\right)+\frac{\gamma_{1} \Delta}{8 M^{3}}\left(\frac{\partial}{\partial M}-\frac{\partial}{\partial \Delta}\right)^{2}\left(\alpha_{1} M^{2} \Delta^{2}\right)+\frac{1}{8 M^{3}}\left(\frac{\partial}{\partial M}-\frac{\partial}{\partial \Delta}\right)\left(\alpha_{1} M \Delta^{3} \gamma_{1}\right) \\
& -\frac{\gamma_{1} \Delta}{8 M^{4}}\left(\frac{\partial}{\partial M}-\frac{\partial}{\partial \Delta}\right)\left(\alpha_{1} M^{2} \Delta^{2}\right), \\
\gamma_{d}^{(5)}= & -\frac{1}{2 M} \frac{\partial}{\partial M} \frac{1}{2(M+\Delta)} \frac{\partial}{\partial \Delta}\left(\alpha_{1} M \Delta^{3} \gamma_{1}\right)+\frac{\gamma_{1} \Delta}{2 M^{2}} \frac{\partial}{\partial M} \frac{1}{2(M+\Delta)} \frac{\partial}{\partial \Delta}\left(\alpha_{1} M^{2} \Delta^{2}\right)-2 \frac{M+\Delta}{M} \gamma_{d}^{(3)},
\end{aligned}
$$

and 


$$
\begin{aligned}
& \Gamma_{d_{1}}^{(1)}=-\frac{32}{3} \tilde{c}_{1}, \quad \Gamma_{d_{2}}^{(1)}=\frac{16}{9}\left(15 \tilde{c}_{0}-2 \tilde{c}_{1}\right), \quad \Gamma_{d_{3}}^{(1)}=8 \tilde{c}_{1}, \quad \Gamma_{d_{4}}^{(1)}=\frac{88}{9}\left(2 \tilde{c}_{0}-\tilde{c}_{1}\right), \\
& \Gamma_{d_{1}}^{(2)}=\frac{40}{3} c_{1}, \quad \Gamma_{d_{2}}^{(2)}=\frac{16}{9}\left(15 c_{0}-2 c_{1}\right), \quad \Gamma_{d_{3}}^{(2)}=0, \quad \Gamma_{d_{4}}^{(2)}=\frac{88}{9}\left(2 c_{0}-c_{1}\right), \\
& \Gamma_{d_{1}}^{(3)}=-\frac{32}{3} \tilde{c}_{1}^{2}, \quad \Gamma_{d_{2}}^{(3)}=-\frac{64}{3}\left(2 \tilde{c}_{0}-\tilde{c}_{1}\right) \tilde{c}_{1}, \quad \Gamma_{d_{3}}^{(3)}=32 \tilde{c}_{1}^{2}, \quad \Gamma_{d_{4}}^{(3)}=\frac{64}{3}\left(16 \tilde{c}_{0}^{2}-10 \tilde{c}_{1} \tilde{c}_{0}+\tilde{c}_{1}^{2}\right), \\
& \Gamma_{d_{1}}^{(4)}=\frac{256}{3} c_{1}^{2}, \quad \Gamma_{d_{2}}^{(4)}=\frac{512}{3}\left(2 c_{0}-c_{1}\right) c_{1}, \quad \Gamma_{d_{3}}^{(4)}=0, \quad \Gamma_{d_{4}}^{(4)}=\frac{256}{3}\left(2 c_{0}-c_{1}\right)^{2}, \\
& \Gamma_{d_{1}}^{(5)}=-\frac{32}{3} c_{1} \tilde{c}_{1}, \quad \Gamma_{d_{2}}^{(5)}=-\frac{64}{3}\left(-8 c_{1} \tilde{c}_{0}+\left(c_{0}+2 c_{1}\right) \tilde{c}_{1}\right), \quad \Gamma_{d_{3}}^{(5)}=0, \quad \Gamma_{d_{4}}^{(5)}=\frac{32}{3}\left(2 c_{0}-c_{1}\right)\left(16 \tilde{c}_{0}-5 \tilde{c}_{1}\right) .
\end{aligned}
$$

We turn to the conventional counting ansatz (38), for which the third- and fourth-order contributions to the $D$-meson polarization tensor are already given with (39), (40). The fifth-order term is

$$
\begin{aligned}
\bar{\Pi}_{H \in\left[0^{-}\right]}^{\text {bubble-5 }}= & \sum_{Q \in[8]} \sum_{R \in\left[1^{-}\right]}\left(\frac{G_{Q R}^{(H)}}{8 \pi f}\right)^{2}\left\{\left[-\frac{3}{4}\left(\Pi_{H}^{(3)}-\Pi_{R}^{(3)}\right) m_{Q}^{2}-\frac{\Delta}{2 M}\left(m_{Q}^{4}+2 m_{Q}^{2}\left(2 \Pi_{H}^{(2)}-\Pi_{R}^{(2)}\right)+\left(\Pi_{H}^{(2)}-\Pi_{R}^{(2)}\right)^{2}\right)\right] \log \frac{m_{Q}^{2}}{M^{2}}\right. \\
& -\left(\Pi_{H}^{(3)}-\Pi_{R}^{(3)}\right) m_{Q}^{2}+\frac{\Delta}{8 M}\left(14 m_{Q}^{4}-m_{Q}^{2}\left(3 \Delta^{2}-16 \Pi_{H}^{(2)}+36 \Pi_{R}^{(2)}\right)-10\left(\Pi_{H}^{(2)}-\Pi_{R}^{(2)}\right)^{2}\right)-\frac{\Delta_{Q}}{M}\left(\log \left(\Delta+\Delta_{Q}\right)\right. \\
& \left.-\log \left(\Delta-\Delta_{Q}\right)\right)\left[-\frac{3}{2} \Delta M\left(\Pi_{H}^{(3)}-\Pi_{R}^{(3)}\right)+\frac{3}{4} \Delta_{Q}^{4}+\frac{1}{2} \Delta_{Q}^{2}\left(3 \Pi_{R}^{(2)}-5 \Pi_{H}^{(2)}\right)+\frac{3}{4}\left(\Pi_{H}^{(2)}-\Pi_{R}^{(2)}\right)^{2}\right. \\
& \left.+\frac{3}{8} m_{Q}^{2}\left(\Delta_{Q}^{2}+2\left(\Pi_{R}^{(2)}-3 \Pi_{H}^{(2)}\right)+\frac{\left(\Pi_{H}^{(2)}-\Pi_{R}^{(2)}\right)^{2}}{\Delta_{Q}^{2}}\right)\right] \\
& +\left[\frac{3 \Delta^{2}}{2}\left(\Pi_{H}^{(3)}-\Pi_{R}^{(3)}\right)-\frac{\Delta}{4 M}\left(3 \Delta_{Q}^{4}-2 m_{Q}^{4}-2\left(\Delta^{2}+m_{Q}^{2}\right)\left(\Pi_{H}^{(2)}+\Pi_{R}^{(2)}\right)\right.\right. \\
& \left.\left.\left.+\left(8 \Delta^{2}+\Pi_{R}^{(2)}-\Pi_{H}^{(2)}\right)\left(\Pi_{R}^{(2)}-\Pi_{H}^{(2)}\right)\right)\right] \log \frac{m_{Q}^{2}}{4 \Delta^{2}}\right\}
\end{aligned}
$$

It remains to specify the fifth-order term with respect to the novel counting ansatz (41). We find

$$
\begin{aligned}
\bar{\Pi}_{H \in\left[0^{-}\right]}^{\text {bubble-5 }}= & \sum_{\substack{Q \in[8] \\
R \in\left[\left[^{3}\right]\right.}}\left(\frac{m_{Q}}{4 \pi f} G_{Q R}^{(H)}\right)^{2}\left\{-\frac{\alpha_{1}}{4} \frac{M^{2}}{2 M+\Delta} \frac{\Delta^{3}}{4 M^{2}} \log \frac{4 \Delta^{2}}{(M+\Delta)^{2}}+\frac{\alpha_{1}}{8} \frac{\Delta^{2}}{m_{Q}^{2}}\left(M_{R}-M_{H}-\Delta_{H}\right)^{2} \frac{M}{M+\Delta} \frac{\partial}{\partial \Delta}\left(\gamma_{1} \Delta\right)\right. \\
& +\frac{M_{H}}{4}\left[\left(\alpha_{1}-\alpha_{2}\right)\left(\frac{2 M+\Delta}{2 M} \frac{m_{Q}^{2}}{\Delta_{H}^{2}}\left(M_{R}-M_{H}\right) \log \frac{m_{Q}^{2}}{M_{R}^{2}}-\left(\delta_{1}-\gamma_{1}\right) \frac{\Delta_{Q}^{2}}{\Delta_{H}}-\delta_{1} \frac{\Delta_{Q}^{2}}{\Delta_{H}^{2}}\left(M_{R}-M_{H}-\Delta_{H}\right)\right)\right. \\
& -\frac{\beta_{11}}{M_{H}^{2}}\left(\left(M_{R}-M_{H}\right)^{3} \log \frac{m_{Q}^{2}}{M_{R}^{2}}+\Delta_{Q}^{3}\left[\log \left(M_{R}-M_{H}+\Delta_{Q}\right)-\log \left(M_{R}-M_{H}-\Delta_{Q}\right)\right]\right) \\
& \left.+\frac{m_{Q}^{2} \Delta_{Q}^{2}}{\Delta_{H}^{3}}\left(\left(\alpha_{2}-\alpha_{1}\right)\left(\delta_{2}+\delta_{3} \log \frac{m_{Q}^{2}}{M_{R}^{2}}\right)-\alpha_{1}\left(\delta_{4}+\delta_{5} \log \frac{m_{Q}^{2}}{M_{R}^{2}}\right)\right)\right] \\
& +\frac{M_{H}}{8}\left(M_{R}-M_{H}-\Delta_{H}\right)^{2}\left[\beta_{9} \frac{\Delta_{Q}^{2}}{m_{Q}^{2} \Delta_{H}}-\beta_{10} \frac{\Delta_{Q}^{2}}{m_{Q}^{2} \Delta_{H}^{2}}\left(M_{R}-M_{B}\right)-\frac{\beta_{6}}{m_{Q}^{2} \Delta_{H}^{2}}\left(\left(M_{R}-M_{H}\right)\left(\Delta_{Q}^{2}-\frac{m_{Q}^{2}}{2}\right) \log \frac{m_{Q}^{2}}{M_{R}^{2}}\right.\right. \\
& \left.\left.\left.+\Delta_{Q}^{3}\left[\log \left(M_{R}-M_{H}+\Delta_{Q}\right)-\log \left(M_{R}-M_{H}-\Delta_{Q}\right)\right]\right)+\left(-\beta_{7} \frac{\Delta_{Q}^{2}}{\Delta_{H}^{3}}+\beta_{8} \frac{m_{Q}^{2}}{\Delta_{H}^{3}} \log \frac{m_{Q}^{2}}{M_{R}^{2}}\right)\right]\right\} .
\end{aligned}
$$

\section{APPENDIX B}

In this appendix, we collect all dimensionless coefficients that are needed in the various power-counting decompositions of the renormalized loop function (16). Here, we focus on the vector $D$ mesons for which we find 


$$
\begin{aligned}
& \tilde{\alpha}_{1}=\frac{(2 M+\Delta)^{2}}{4 M^{2}}, \quad \tilde{\alpha}_{2}=\frac{2 M^{2}+2 \Delta M+\Delta^{2}}{2 M^{2}}, \quad \tilde{\alpha}_{3}=1, \\
& \tilde{\gamma}_{1}=-\frac{M(2 M+\Delta)}{(M+\Delta)^{2}} \log \frac{\Delta(2 M+\Delta)}{M^{2}}, \\
& \tilde{\gamma}_{2}=\frac{M}{2 M+\Delta}+M \frac{2 M^{2}+2 \Delta M+\Delta^{2}}{(2 M+\Delta)(M+\Delta)^{2}} \log \frac{\Delta(2 M+\Delta)}{M^{2}}, \quad \tilde{\gamma}_{3}=-\frac{M}{2 M+\Delta}, \\
& \tilde{\gamma}_{4}=-\frac{M(M+\Delta)^{2}}{2(2 M+\Delta)^{3}}+\frac{2 M^{3}}{(2 M+\Delta)^{3}} \log \frac{\Delta(2 M+\Delta)}{M^{2}}, \quad \tilde{\gamma}_{5}=-\frac{M^{3}}{(2 M+\Delta)^{3}}, \\
& \tilde{\delta}_{1}=\tilde{\gamma}_{1}+\frac{M(2 M+\Delta)}{(M+\Delta)^{2}} \log \frac{2 \Delta}{M} \\
& \tilde{\delta}_{2}=\tilde{\gamma}_{2}-\frac{M\left(2 M^{2}+2 \Delta M+\Delta^{2}\right)}{(2 M+\Delta)(M+\Delta)^{2}} \log \frac{2 \Delta}{M+\Delta}-\frac{M(2 M+\Delta)}{4(M+\Delta)^{2}}-2\left(\tilde{\delta}_{3}-\tilde{\gamma}_{3}\right) \log \frac{M+\Delta}{M}, \\
& \tilde{\delta}_{3}=\tilde{\gamma}_{3}+\frac{M\left(2 M^{2}+2 \Delta M+\Delta^{2}\right)}{2(M+\Delta)^{2}(2 M+\Delta)}, \quad \tilde{\delta}_{5}=0, \\
& \tilde{\delta}_{4}=\tilde{\gamma}_{4}-\frac{2 M^{3}}{(2 M+\Delta)^{3}} \log \frac{2 \Delta}{M+\Delta}+\frac{M\left(4 M^{2}+4 \Delta M+5 \Delta^{2}\right)}{32(M+\Delta)^{2}(2 M+\Delta)}-2\left(\tilde{\delta}_{5}-\tilde{\gamma}_{5}\right) \log \frac{M+\Delta}{M} \text {, } \\
& \tilde{\delta}_{6}=\frac{2 M+\Delta}{2 M} \frac{\partial}{\partial \Delta} \frac{2(M+\Delta)}{2 M+\Delta} \Delta\left(\tilde{\gamma}_{1}-\tilde{\delta}_{1}\right)+\tilde{\delta}_{1}, \quad \tilde{\delta}_{7}=\tilde{\gamma}_{2}+\frac{1}{2}\left(\tilde{\gamma}_{1}-\tilde{\delta}_{1}\right) \frac{\Delta^{2}}{(2 M+\Delta)^{2}}, \\
& \tilde{\beta}_{1}=\frac{M+\Delta}{M} \frac{\Delta \partial}{\partial \Delta} \tilde{\alpha}_{1} \frac{(2 M+\Delta) M^{2}}{2(M+\Delta)^{3}}, \quad \tilde{\beta}_{2}=\Delta^{2} \frac{\partial}{\partial \Delta} \frac{\tilde{\alpha}_{1} \tilde{\delta}_{2}}{\Delta}, \quad \tilde{\beta}_{3}=\Delta^{2} \frac{\partial}{\partial \Delta} \frac{\tilde{\alpha}_{1} \tilde{\delta}_{3}}{\Delta},
\end{aligned}
$$

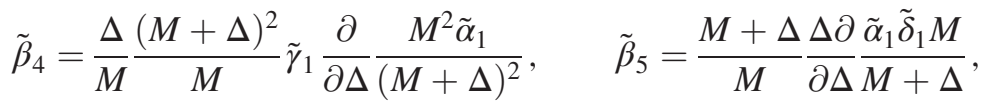

$$
\begin{aligned}
& \tilde{\beta}_{6}=D_{\Delta \Delta} \frac{(2 M+\Delta) M^{2}}{2(M+\Delta)^{3}} \tilde{\alpha}_{1}, \quad \tilde{\beta}_{7}=\frac{\Delta}{M+\Delta} D_{\Delta \Delta} \frac{M}{\Delta} \tilde{\alpha}_{1} \tilde{\delta}_{2}, \\
& \tilde{\beta}_{8}=\frac{\Delta}{M+\Delta} D_{\Delta \Delta} \frac{M}{\Delta} \tilde{\alpha}_{1} \tilde{\delta}_{3}, \quad \tilde{\beta}_{9}=\tilde{\gamma}_{1} \frac{M+\Delta}{M} D_{\Delta \Delta} \frac{M^{2}}{(M+\Delta)^{2}} \tilde{\alpha}_{1}, \\
& \tilde{\beta}_{10}=D_{\Delta \Delta} \frac{M}{M+\Delta} \tilde{\alpha}_{1} \tilde{\delta}_{1}, \quad \tilde{\beta}_{11}=-\frac{1}{4} \tilde{\alpha}_{1} \frac{M}{2 M+\Delta}+\left(\tilde{\alpha}_{1}-\tilde{\alpha}_{2}\right) \frac{(2 M+\Delta) M}{2 \Delta^{2}}, \\
& \text { with } \quad D_{\Delta \Delta}=\frac{(M+\Delta)^{2}}{M^{2}}\left(\frac{\Delta^{2} \partial^{2}}{\partial \Delta \partial \Delta}+\frac{2 \Delta}{M+\Delta} \frac{\Delta \partial}{\partial \Delta}\right) \text {. }
\end{aligned}
$$

While the $\tilde{\alpha}_{i}$ characterize the chiral expansion of the coefficients in front of $\bar{I}_{Q R}$ and $\bar{I}_{Q}$ in (16), the $\tilde{\gamma}_{i}$ and $\tilde{\delta}_{i}$ follow from a chiral expansion of $\bar{I}_{Q R}$ with $M_{H}=M+\Delta$ and $M_{R}=M$ and $m_{Q}<\Delta$. The coefficients $\tilde{\beta}_{i}$ are required in (44), (45), (B5).

We turn to the chiral domain (34), in which the bubble-loop contributions to the $D^{*}$-meson masses generate a renormalization of the low-energy parameters $\tilde{d}_{i}$. Such terms are proportional to the product of two quark masses (9). We provide detailed results with

$$
\begin{aligned}
\Pi_{H}^{(4-\chi)} & \rightarrow \sum_{Q \in[8]} \sum_{R \in\left[0^{-}\right]}\left(\frac{G_{Q R}^{H}}{8 \pi f}\right)^{2}\left\{\tilde{\gamma}_{d}^{(1)} m_{Q}^{2} \Pi_{R}^{(2)}+\tilde{\gamma}_{d}^{(2)} m_{Q}^{2} \Pi_{H}^{(2)}+\tilde{\gamma}_{d}^{(3)} \Pi_{R}^{(2)} \Pi_{R}^{(2)}+\tilde{\gamma}_{d}^{(4)} \Pi_{H}^{(2)} \Pi_{H}^{(2)}+\tilde{\gamma}_{d}^{(5)} \Pi_{R}^{(2)} \Pi_{H}^{(2)}\right\}, \\
\tilde{d}_{i} & \rightarrow \frac{1}{4} g_{P}^{2} \sum_{k=1}^{5} \frac{\tilde{\Gamma}_{d_{i}}^{(k)}}{(4 \pi f)^{2}} \tilde{\gamma}_{d}^{(k)},
\end{aligned}
$$

with 


$$
\begin{aligned}
\tilde{\gamma}_{d}^{(1)}= & \frac{\Delta}{6 M}\left[\frac{\partial}{\partial M}\left(\tilde{\alpha}_{2} M \tilde{\gamma}_{1}-\tilde{\alpha}_{1} M \tilde{\gamma}_{2}\right)-\frac{1}{M} \tilde{\gamma}_{1} \frac{\partial}{\partial M}\left(\tilde{\alpha}_{2} M^{2}\right)\right]-\frac{M+\Delta}{M} \tilde{\gamma}_{d}^{(2)}, \\
\tilde{\gamma}_{d}^{(2)}= & \frac{M}{6(M+\Delta)}\left[\frac{\partial}{\partial \Delta}\left(\tilde{\alpha}_{2} \Delta \tilde{\gamma}_{1}-\tilde{\alpha}_{1} \Delta \tilde{\gamma}_{2}\right)-\Delta \tilde{\gamma}_{1} \frac{\partial}{\partial \Delta} \tilde{\alpha}_{2}\right], \\
\tilde{\gamma}_{d}^{(3)}= & -\frac{1}{24 M^{2}}\left(\frac{\partial}{\partial M}-\frac{\partial}{\partial \Delta}\right)^{2}\left(\tilde{\alpha}_{1} M \Delta^{3} \tilde{\gamma}_{1}\right)+\frac{\tilde{\gamma}_{1} \Delta}{24 M^{3}}\left(\frac{\partial}{\partial M}-\frac{\partial}{\partial \Delta}\right)^{2}\left(\tilde{\alpha}_{1} M^{2} \Delta^{2}\right) \\
& +\frac{1}{24 M^{3}}\left(\frac{\partial}{\partial M}-\frac{\partial}{\partial \Delta}\right)\left(\tilde{\alpha}_{1} M \Delta^{3} \tilde{\gamma}_{1}\right)-\frac{\tilde{\gamma}_{1} \Delta}{24 M^{4}}\left(\frac{\partial}{\partial M}-\frac{\partial}{\partial \Delta}\right)\left(\tilde{\alpha}_{1} M^{2} \Delta^{2}\right), \\
\tilde{\gamma}_{d}^{(4)}= & -\frac{M}{12(M+\Delta)^{2}}\left(\frac{\partial \tilde{\alpha}_{1} \Delta^{2}}{\partial \Delta}\right)\left(\frac{\partial \tilde{\gamma}_{1} \Delta}{\partial \Delta}\right)-\frac{\tilde{\alpha}_{1} \Delta^{2} M}{12(M+\Delta)} \frac{\partial}{\partial \Delta}\left[\frac{1}{2(M+\Delta)}\left(\frac{\partial \tilde{\gamma}_{1} \Delta}{\partial \Delta}\right)\right], \\
\tilde{\gamma}_{d}^{(5)}= & -\frac{1}{6 M} \frac{\partial}{\partial M} \frac{1}{2(M+\Delta)} \frac{\partial}{\partial \Delta}\left(\tilde{\alpha}_{1} M \Delta^{3} \tilde{\gamma}_{1}\right)+\frac{\tilde{\gamma}_{1} \Delta}{6 M^{2}} \frac{\partial}{\partial M} \frac{\partial}{2(M+\Delta)}\left(\tilde{\alpha}_{1} M^{2} \Delta^{2}\right)-2 \frac{M+\Delta}{\partial \tilde{\gamma}_{d}^{(4)}},
\end{aligned}
$$

where the other $\Gamma_{\tilde{d}_{i}}^{(k)}$ with $k=1,2, \ldots, 5$ follow from the corresponding $\Gamma_{d_{i}}^{(k)}$ in (A4) upon the interchange $c_{i} \leftrightarrow \tilde{c}_{i}$.

We turn to the conventional counting ansatz (38), for which the third- and fourth-order contributions to the $D^{*}$-meson polarization tensor are already given with (39), (40). The fifth-order term is

$$
\begin{aligned}
\bar{\Pi}_{H \in\left[1^{-}\right]}^{\text {bubble- }}= & \sum_{Q \in[8]} \sum_{R \in\left[1^{-}\right]}\left(\frac{G_{Q R}^{(H)}}{8 \pi f}\right)^{2} \frac{1}{12}\left\{-8\left(\Pi_{H}^{(3)}-\Pi_{R}^{(3)}\right) m_{Q}^{2}+\frac{4 \Delta}{M} m_{Q}^{2}\left(2 m_{Q}^{2}-3 \Pi_{R}^{(2)}+3 \Pi_{H}^{(2)}\right)-6\left(\Pi_{H}^{(3)}-\Pi_{R}^{(3)}\right) m_{Q}^{2} \log \frac{m_{Q}^{2}}{M^{2}}\right. \\
& \left.+\pi \frac{m_{Q}}{M}\left[3 m_{Q}^{4}+2 m_{Q}^{2}\left(\Pi_{H}^{(2)}-3 \Pi_{R}^{(2)}\right)+3\left(\Pi_{R}^{(2)}-\Pi_{H}^{(2)}\right)^{2}\right]\right\} \\
& +\sum_{Q \in[8]} \sum_{R \in\left[0^{-}\right]}\left(\frac{G_{Q R}^{(H)}}{8 \pi f}\right)^{2} \frac{1}{3}\left\{\left[-\frac{3}{4}\left(\Pi_{H}^{(3)}-\Pi_{R}^{(3)}\right) m_{Q}^{2}+\frac{\Delta}{2 M}\left(m_{Q}^{4}+2 m_{Q}^{2}\left(2 \Pi_{H}^{(2)}-\Pi_{R}^{(2)}\right)+\left(\Pi_{H}-\Pi_{R}\right)^{2}\right)\right] \log \frac{m_{Q}^{2}}{M^{2}}\right. \\
& -\left(\Pi_{H}^{(3)}-\Pi_{R}^{(3)}\right) m_{Q}^{2}+\frac{\Delta}{8 M}\left[-6 m_{Q}^{4}+m_{Q}^{2}\left(3 \Delta^{2}-4 \Pi_{H}^{(2)}+24 \Pi_{R}^{(2)}\right)+10\left(\Pi_{H}^{(2)}-\Pi_{R}^{(2)}\right)^{2}\right] \\
& +\frac{\Delta_{Q}}{M}\left(\log \left(-\Delta-\Delta_{Q}\right)-\log \left(-\Delta+\Delta_{Q}\right)\right)\left[\frac{3}{2} \Delta M\left(\Pi_{H}^{(3)}-\Pi_{R}^{(3)}\right)+\frac{3}{4} \Delta_{Q}^{4}+\frac{1}{2} \Delta_{Q}^{2}\left(3 \Pi_{R}^{(2)}-5 \Pi_{H}^{(2)}\right)\right. \\
& \left.+\frac{3}{4}\left(\Pi_{H}^{(2)}-\Pi_{R}^{(2)}\right)^{2}+\frac{3}{8} m_{Q}^{2}\left(\Delta_{Q}^{2}+2\left(\Pi_{R}^{(2)}-3 \Pi_{H}^{(2)}\right)+\frac{\left(\Pi_{H}^{(2)}-\Pi_{R}^{(2)}\right)^{2}}{\Delta_{Q}^{2}}\right)\right] \\
& +\left[\frac{3}{2} \Delta^{2}\left(\Pi_{H}^{(3)}-\Pi_{R}^{(3)}\right)+\frac{\Delta}{4 M}\left(3 \Delta_{Q}^{4}-2 m_{Q}^{4}-2\left(\Delta^{2}+m_{Q}^{2}\right)\left(\Pi_{H}^{(2)}+\Pi_{R}^{(2)}\right)\right.\right. \\
& \left.\left.\left.+\left(8 \Delta^{2}+\Pi_{R}^{(2)}-\Pi_{H}^{(2)}\right)\left(\Pi_{R}^{(2)}-\Pi_{H}^{(2)}\right)\right)\right] \log \frac{m_{Q}^{2}}{4 \Delta^{2}}\right\} .
\end{aligned}
$$

It remains to specify the fifth-order term with respect to the novel counting ansatz (41). We find 


$$
\begin{aligned}
& \bar{\Pi}_{H \in\left[1^{-}\right]}^{\text {bubble-5 }}=\sum_{\substack{Q \in[8] \\
R \in\left[1^{-}\right]}}\left(\frac{m_{Q R}}{4 \pi f} G_{Q R}^{(H)}\right)^{2} \frac{1}{3}\left\{\frac{3 \pi}{16} \frac{m_{Q}^{3}}{M_{H}}-\frac{m_{Q}^{4}}{M_{H}^{2}}\left(\frac{1}{6}-\frac{1}{8} \log \frac{m_{Q}}{M_{R}}\right)+\left(M_{R}-M_{H}\right)^{2}\left(\frac{\pi}{4} \frac{M_{H}}{m_{Q}}+1+\frac{3}{2} \log \frac{m_{Q}}{M_{R}}\right)\right\} \\
& +\sum_{\substack{Q \in[8] \\
R \in\left[0^{-}\right]}}\left(\frac{m_{Q}}{4 \pi f} G_{Q R}^{(H)}\right)^{2}\left\{\frac{\tilde{\alpha}_{1}}{12} \frac{M^{2}}{(2 M+\Delta)} \frac{\Delta^{3}}{4(M+\Delta)^{2}} \log \frac{4 \Delta^{2}}{M^{2}}-\frac{\tilde{\alpha}_{1}}{24} \frac{\Delta^{2}}{m_{Q}^{2}}\left(M_{R}-M_{H}+\Delta_{H}\right)^{2} \frac{M+\Delta}{M} \frac{\partial}{\partial \Delta}\left(\tilde{\gamma}_{1} \Delta\right)\right. \\
& +\frac{M_{H}}{12} \frac{M}{M+\Delta}\left[-\left(\tilde{\alpha}_{1}-\tilde{\alpha}_{2}\right)\left(\frac{M(2 M+\Delta)}{2(M+\Delta)^{2}} \frac{m_{Q}^{2}}{\Delta_{H}^{2}}\left(M_{H}-M_{R}\right) \log \frac{m_{Q}^{2}}{M_{R}^{2}}+\left(\tilde{\delta}_{1}-\tilde{\gamma}_{1}\right) \frac{\Delta_{Q}^{2}}{\Delta_{H}}-\tilde{\delta}_{1} \frac{\Delta_{Q}^{2}}{\Delta_{H}^{2}}\left(M_{R}-M_{H}+\Delta_{H}\right)\right)\right. \\
& +\frac{\tilde{\beta}_{11}}{M_{H}^{2}}\left(\left(M_{H}-M_{R}\right)^{3} \log \frac{m_{Q}^{2}}{M_{R}^{2}}+\Delta_{Q}^{3}\left[\log \left(M_{R}-M_{H}-\Delta_{Q}\right)-\log \left(M_{R}-M_{H}+\Delta_{Q}\right)\right]\right) \\
& \left.+\frac{m_{Q}^{2} \Delta_{Q}^{2}}{\Delta_{H}^{3}}\left(\left(\tilde{\alpha}_{2}-\tilde{\alpha}_{1}\right)\left(\tilde{\delta}_{2}+\tilde{\delta}_{3} \log \frac{m_{Q}^{2}}{M_{R}^{2}}\right)-\tilde{\alpha}_{1}\left(\tilde{\delta}_{4}+\tilde{\delta}_{5} \log \frac{m_{Q}^{2}}{M_{R}^{2}}\right)\right)\right] \\
& +\frac{M_{H}}{24}\left(M_{R}-M_{H}+\Delta_{H}\right)^{2}\left[\tilde{\beta}_{9} \frac{\Delta_{Q}^{2}}{m_{Q}^{2} \Delta_{H}}-\tilde{\beta}_{10} \frac{\Delta_{Q}^{2}}{m_{Q}^{2} \Delta_{H}^{2}}\left(M_{H}-M_{R}\right)\right. \\
& +\frac{\tilde{\beta}_{6}}{m_{Q}^{2} \Delta_{H}^{2}}\left(\left(M_{H}-M_{R}\right)\left(\Delta_{Q}^{2}-\frac{m_{Q}^{2}}{2}\right) \log \frac{m_{Q}^{2}}{M_{R}^{2}}+\Delta_{Q}^{3}\left[\log \left(M_{R}-M_{H}-\Delta_{Q}\right)-\log \left(M_{R}-M_{H}+\Delta_{Q}\right)\right]\right) \\
& \left.\left.+\left(-\tilde{\beta}_{7} \frac{\Delta_{Q}^{2}}{\Delta_{H}^{3}}+\tilde{\beta}_{8} \frac{m_{Q}^{2}}{\Delta_{H}^{3}} \log \frac{m_{Q}^{2}}{M_{R}^{2}}\right)\right]\right\} \text {, with } \quad m_{Q R}^{2}=m_{Q}^{2}-\left(M_{R}-M_{H}\right)^{2} .
\end{aligned}
$$

\begin{tabular}{|c|c|c|c|c|c|c|c|}
\hline$a m_{\pi}$ & $a m_{K}$ & $a \mu_{c}$ & Discr. & $a m_{D}$ & $a m_{D_{s}}$ & $a m_{D^{*}}$ & $a m_{D_{s}^{*}}$ \\
\hline $0.0703(4)$ & $0.1697(3)$ & $\begin{array}{l}0.2230 \\
0.1919 \\
0.2230 \\
0.1919\end{array}$ & $\begin{array}{l}( \pm, \mp) \\
( \pm, \mp) \\
( \pm, \pm) \\
( \pm, \pm)\end{array}$ & $\begin{array}{l}0.6655(12) \\
0.6072(11) \\
0.6706(15) \\
0.6123(14)\end{array}$ & $\begin{array}{l}0.6981(4) \\
0.6402(3) \\
0.7035(5) \\
0.6460(4)\end{array}$ & $\begin{array}{l}0.7161(18) \\
0.6621(18) \\
0.7078(24) \\
0.6536(23)\end{array}$ & $\begin{array}{l}0.7456(10) \\
0.6923(10) \\
0.7430(10) \\
0.6898(10)\end{array}$ \\
\hline $0.0806(3)$ & $0.1738(5)$ & $\begin{array}{l}0.2227 \\
0.1727 \\
0.2227 \\
0.1727\end{array}$ & $\begin{array}{l}( \pm, \mp) \\
( \pm, \mp) \\
( \pm, \pm) \\
( \pm, \pm)\end{array}$ & $\begin{array}{l}0.6661(19) \\
0.5712(14) \\
0.6721(22) \\
0.5775(17)\end{array}$ & $\begin{array}{l}0.6983(4) \\
0.6041(4) \\
0.7037(5) \\
0.6102(4)\end{array}$ & $\begin{array}{l}0.7209(26) \\
0.6325(25) \\
0.7209(20) \\
0.6335(23)\end{array}$ & $\begin{array}{l}0.7452(12) \\
0.6586(11) \\
0.7452(10) \\
0.6587(10)\end{array}$ \\
\hline $0.0975(3)$ & $0.1768(3)$ & $\begin{array}{l}0.2230 \\
0.1727 \\
0.2230 \\
0.1727\end{array}$ & $\begin{array}{l}( \pm, \mp) \\
( \pm, \mp) \\
( \pm, \pm) \\
( \pm, \pm)\end{array}$ & $\begin{array}{l}0.6666(16) \\
0.5720(12) \\
0.6713(13) \\
0.5770(12)\end{array}$ & $\begin{array}{l}0.6980(5) \\
0.6036(4) \\
0.7033(5) \\
0.6098(4)\end{array}$ & $\begin{array}{l}0.7183(23) \\
0.6308(24) \\
0.7169(19) \\
0.6290(22)\end{array}$ & $\begin{array}{l}0.7458(13) \\
0.6587(13) \\
0.7451(8) \\
0.6579(11)\end{array}$ \\
\hline $0.1074(5)$ & $0.2133(4)$ & $\begin{array}{l}0.2230 \\
0.1727 \\
0.2230 \\
0.1727\end{array}$ & $\begin{array}{l}( \pm, \mp) \\
( \pm, \mp) \\
( \pm, \pm) \\
( \pm, \pm)\end{array}$ & $\begin{array}{l}0.8473(10) \\
0.7501(8) \\
0.8588(16) \\
0.7629(14)\end{array}$ & $\begin{array}{l}0.8780(5) \\
0.7827(4) \\
0.8922(7) \\
0.7978(6)\end{array}$ & $\begin{array}{l}0.9140(31) \\
0.8262(29) \\
0.9112(25) \\
0.8224(24)\end{array}$ & $\begin{array}{l}0.9474(10) \\
0.8601(9) \\
0.9443(10) \\
0.8566(10)\end{array}$ \\
\hline $0.1549(2)$ & $0.2279(2)$ & $\begin{array}{l}0.2230 \\
0.1727 \\
0.2230 \\
0.1727\end{array}$ & $\begin{array}{l}( \pm, \mp) \\
( \pm, \mp) \\
( \pm, \pm) \\
( \pm, \pm)\end{array}$ & $\begin{array}{l}0.8543(5) \\
0.7549(5) \\
0.8666(8) \\
0.7683(7)\end{array}$ & $\begin{array}{l}0.8824(3) \\
0.7841(3) \\
0.8961(4) \\
0.7991(3)\end{array}$ & $\begin{array}{l}0.9268(11) \\
0.8362(11) \\
0.9218(11) \\
0.8322(17)\end{array}$ & $\begin{array}{l}0.9536(7) \\
0.8637(8) \\
0.9500(6) \\
0.8597(7)\end{array}$ \\
\hline $0.1935(4)$ & $0.2430(4)$ & $\begin{array}{l}0.2230 \\
0.1727 \\
0.2230 \\
0.1727\end{array}$ & $\begin{array}{l}( \pm, \mp) \\
( \pm, \mp) \\
( \pm, \pm) \\
( \pm, \pm)\end{array}$ & $\begin{array}{l}0.8559(8) \\
0.7600(11) \\
0.8690(8) \\
0.7763(7)\end{array}$ & $\begin{array}{l}0.8784(5) \\
0.7850(4) \\
0.8928(5) \\
0.8007(5)\end{array}$ & $\begin{array}{l}0.9309(18) \\
0.8443(18) \\
0.9273(14) \\
0.8413(14)\end{array}$ & $\begin{array}{l}0.9521(13) \\
0.8669(13) \\
0.9484(11) \\
0.8629(11)\end{array}$ \\
\hline
\end{tabular}

TABLE XXI. Masses for the $D$ mesons in units of the lattice scale $a$. The values in the table were provided to us by the authors of Ref. [20]. "Discr." stands for the various choices of the discretization scheme (see, e.g., [20]). 
TABLE XXII. Masses for the $D$ mesons in units of the lattice scale $a$. The values in the table were provided to us by the authors of Ref. [20]. "Discr." stands for the various choices of the discretization scheme (see, e.g., [20]).

\begin{tabular}{|c|c|c|c|c|c|c|c|}
\hline$a m_{\pi}$ & $a m_{K}$ & $a \mu_{c}$ & Discr. & $a m_{D}$ & $a m_{D_{s}}$ & $a m_{D^{*}}$ & $a m_{D_{s}^{*}}$ \\
\hline \multirow[t]{4}{*}{$0.1240(4)$} & $0.2512(3)$ & 0.2772 & $( \pm, \mp)$ & $0.8979(9)$ & $0.9412(2)$ & $0.9782(16)$ & $1.0225(7)$ \\
\hline & & 0.2270 & $( \pm, \mp)$ & $0.7994(8)$ & $0.8441(2)$ & $0.8880(16)$ & $0.9338(7)$ \\
\hline & & 0.2772 & $( \pm, \pm)$ & $0.9154(14)$ & $0.9610(3)$ & $0.9759(15)$ & $1.0185(7)$ \\
\hline & & 0.2270 & $( \pm, \pm)$ & $0.8181(12)$ & $0.8655(3)$ & $0.8859(15)$ & $0.9289(8)$ \\
\hline \multirow[t]{4}{*}{$0.1412(3)$} & $0.2569(3)$ & 0.2768 & $( \pm, \mp)$ & $0.9002(10)$ & $0.9420(3)$ & $0.9776(20)$ & $1.0213(9)$ \\
\hline & & 0.2389 & $( \pm, \mp)$ & $0.8258(9)$ & $0.8692(3)$ & $0.9104(20)$ & $0.9545(9)$ \\
\hline & & 0.2768 & $( \pm, \pm)$ & $0.9162(13)$ & $0.9623(4)$ & $0.9743(19)$ & $1.0169(9)$ \\
\hline & & 0.2389 & $( \pm, \pm)$ & $0.8433(12)$ & $0.8904(4)$ & $0.9067(18)$ & $0.9501(9)$ \\
\hline \multirow[t]{4}{*}{$0.1440(6)$} & $0.2589(4)$ & 0.2768 & $( \pm, \mp)$ & $0.9006(8)$ & $0.9425(3)$ & $0.9801(23)$ & $1.0252(8)$ \\
\hline & & 0.2389 & $( \pm, \mp)$ & $0.8268(12)$ & $0.8697(2)$ & $0.9153(19)$ & $0.9589(8)$ \\
\hline & & 0.2768 & $( \pm, \pm)$ & $0.9160(13)$ & $0.9627(3)$ & $0.9813(16)$ & $1.0208(7)$ \\
\hline & & 0.2389 & $( \pm, \pm)$ & $0.8432(11)$ & $0.8911(3)$ & $0.9145(15)$ & $0.9544(7)$ \\
\hline \multirow[t]{4}{*}{$0.1988(3)$} & $0.2764(3)$ & 0.2929 & $( \pm, \mp)$ & $0.9327(8)$ & $0.9668(5)$ & $1.0148(17)$ & $1.0496(12)$ \\
\hline & & 0.2299 & $( \pm, \mp)$ & $0.8164(13)$ & $0.8520(4)$ & $0.9092(16)$ & $0.9449(11)$ \\
\hline & & 0.2929 & $( \pm, \pm)$ & $0.9500(12)$ & $0.9879(5)$ & $1.0098(44)$ & $1.0434(20)$ \\
\hline & & 0.2299 & $( \pm, \pm)$ & $0.8358(10)$ & $0.8746(5)$ & $0.9026(41)$ & $0.9381(18)$ \\
\hline
\end{tabular}

[1] T.-M. Yan, H.-Y. Cheng, C.-Y. Cheung, G.-L. Lin, Y. C. Lin, and H.-L. Yu, Phys. Rev. D 46, 1148 (1992); 55, 5851(E) (1997).

[2] R. Casalbuoni, A. Deandrea, N. Di Bartolomeo, R. Gatto, F. Feruglio, and G. Nardulli, Phys. Rep. 281, 145 (1997).

[3] M. F. M. Lutz et al., Nucl. Phys. A948, 93 (2016).

[4] H.-X. Chen, W. Chen, X. Liu, Y.-R. Liu, and S.-L. Zhu, Rep. Prog. Phys. 80, 076201 (2017).

[5] A. V. Manohar and M. B. Wise, Cambridge Monographs on Particle Physics, Nuclear Physics and Cosmology (Cambridge University Press, 2007), Vol. 10, ISBN: 9780521037570.

[6] M. F. M. Lutz and M. Soyeur, Nucl. Phys. A813, 14 (2008).

[7] M. F. M. Lutz, D. Samart, and Y. Yan, Phys. Rev. D 90, 056006 (2014).

[8] E. Kolomeitsev and M. F. M. Lutz, Phys. Lett. B 582, 39 (2004).

[9] J. Hofmann and M. F. M. Lutz, Nucl. Phys. A733, 142 (2004).

[10] L. Liu, K. Orginos, F.-K. Guo, C. Hanhart, and U.-G. Meißner, Phys. Rev. D 87, 014508 (2013).

[11] M. Altenbuchinger, L. S. Geng, and W. Weise, Phys. Rev. D 89, 014026 (2014).

[12] M. Cleven, H. W. Griehammer, F.-K. Guo, C. Hanhart, and U.-G. Meißner, Eur. Phys. J. A 50, 149 (2014).

[13] M.-L. Du, F.-K. Guo, U.-G. Meißner, and D.-L. Yao, Phys. Rev. D 94, 094037 (2016).

[14] F.-K. Guo, P.-N. Shen, H.-C. Chiang, R.-G. Ping, and B.-S. Zou, Phys. Lett. B 641, 278 (2006).

[15] F.-K. Guo, P.-N. Shen, and H.-C. Chiang, Phys. Lett. B 647, 133 (2007).
[16] M. Albaladejo, P. Fernandez-Soler, F.-K. Guo, and J. Nieves, Phys. Lett. B 767, 465 (2017).

[17] S. Aoki et al. (PACS-CS Collaboration), Phys. Rev. D 79, 034503 (2009).

[18] D. Mohler and R. Woloshyn, Phys. Rev. D 84, 054505 (2011).

[19] H. Na, C. T. Davies, E. Follana, G. P. Lepage, and J. Shigemitsu, Phys. Rev. D 86, 054510 (2012).

[20] M. Kalinowski and M. Wagner, Phys. Rev. D 92, 094508 (2015).

[21] K. Cichy, M. Kalinowski, and M. Wagner, Phys. Rev. D 94, 094503 (2016).

[22] G. K. C. Cheung, C. O’Hara, G. Moir, M. Peardon, S. M. Ryan, C.E. Thomas, and D. Tims (Hadron Spectrum Collaboration), J. High Energy Phys. 12 (2016) 089.

[23] G. Moir, M. Peardon, S. M. Ryan, C. E. Thomas, and D. J. Wilson, J. High Energy Phys. 10 (2016) 011.

[24] R. D. Young, D. B. Leinweber, and A. W. Thomas, Prog. Part. Nucl. Phys. 50, 399 (2003).

[25] D. B. Leinweber, A. W. Thomas, and R. D. Young, Phys. Rev. Lett. 92, 242002 (2004).

[26] S. R. Beane, Nucl. Phys. B695, 192 (2004).

[27] D. B. Leinweber, A. W. Thomas, and R. D. Young, Nucl. Phys. A755, 59 (2005).

[28] J. A. McGovern and M. C. Birse, Phys. Rev. D 74, 097501 (2006).

[29] D. Djukanovic, J. Gegelia, and S. Scherer, Eur. Phys. J. A 29, 337 (2006).

[30] M. R. Schindler, D. Djukanovic, J. Gegelia, and S. Scherer, Nucl. Phys. A803, 68 (2008).

[31] J. Hall, D. Leinweber, and R. Young, Phys. Rev. D 82, 034010 (2010). 
[32] A. Semke and M. F. M. Lutz, Nucl. Phys. A778, 153 (2006).

[33] A. Semke and M.F. M. Lutz, Phys. Rev. D 85, 034001 (2012).

[34] M. F. M. Lutz, R. Bavontaweepanya, C. Kobdaj, and K. Schwarz, Phys. Rev. D 90, 054505 (2014).

[35] M. F. M. Lutz, Y. Heo, and X.-Y. Guo, Nucl. Phys. A977, 146 (2018).

[36] F.-K. Guo, C. Hanhart, S. Krewald, and U.-G. Meißner, Phys. Lett. B 666, 251 (2008).

[37] P. J. Ellis and H.-B. Tang, Phys. Rev. C 57, 3356 (1998).

[38] T. Becher and H. Leutwyler, Eur. Phys. J. C 9, 643 (1999).

[39] J. Gegelia and G. Japaridze, Phys. Rev. D 60, 114038 (1999).

[40] M. Lutz, Nucl. Phys. A677, 241 (2000).

[41] M. F. M. Lutz and E. Kolomeitsev, Nucl. Phys. A700, 193 (2002).

[42] G. Passarino and M. J. G. Veltman, Nucl. Phys. B160, 151 (1979).

[43] K. Nakamura et al. (Particle Data Group Collaboration), J. Phys. G 37, 075021 (2010).

[44] M. Banerjee and J. Milana, Phys. Rev. D 52, 6451 (1995).

[45] M. Banerjee and J. Milana, Phys. Rev. D 54, 5804 (1996).

[46] C. Aubin et al., Phys. Rev. Lett. 95, 122002 (2005).

[47] H. Na, C. T. Davies, E. Follana, G. P. Lepage, and J. Shigemitsu, Phys. Rev. D 82, 114506 (2010).

[48] E. Follana, C. Davies, G. Lepage, and J. Shigemitsu (HPQCD and UKQCD Collaborations), Phys. Rev. Lett. 100, 062002 (2008).

[49] A. Bazavov et al. (Fermilab Lattice and MILC Collaborations), Phys. Rev. D 85, 114506 (2012).

[50] R. Dowdall, C. Davies, T. Hammant, and R. Horgan, Phys. Rev. D 86, 094510 (2012).

[51] D. Mohler, C. Lang, L. Leskovec, S. Prelovsek, and R. Woloshyn, Phys. Rev. Lett. 111, 222001 (2013).

[52] G. Moir, M. Peardon, S. M. Ryan, C. E. Thomas, and L. Liu, J. High Energy Phys. 05 (2013) 021.

[53] C. Lang, L. Leskovec, D. Mohler, S. Prelovsek, and R. Woloshyn, Phys. Rev. D 90, 034510 (2014).

[54] A. Bazavov et al. (Fermilab Lattice and MILC Collaborations), Phys. Rev. D 90, 074509 (2014).

[55] S. Aoki et al., Eur. Phys. J. C 77, 112 (2017).

[56] A. X. El-Khadra, A. S. Kronfeld, and P. B. Mackenzie, Phys. Rev. D 55, 3933 (1997).

[57] M. B. Oktay and A. S. Kronfeld, Phys. Rev. D 78, 014504 (2008).

[58] C. Alexandrou, M. Constantinou, S. Dinter, V. Drach, K. Jansen, C. Kallidonis, and G. Koutsou, Phys. Rev. D 88, 014509 (2013).

[59] N. Carrasco et al. (European Twisted Mass Collaboration), Nucl. Phys. B887, 19 (2014).
[60] A. Walker-Loud et al., Phys. Rev. D 79, 054502 (2009).

[61] K. Orginos, D. Toussaint, and R. L. Sugar (MILC Collaboration), Phys. Rev. D 60, 054503 (1999).

[62] K. Orginos and D. Toussaint (MILC Collaboration), Phys. Rev. D 59, 014501 (1998).

[63] C. W. Bernard, T. Burch, K. Orginos, D. Toussaint, T. A. DeGrand, C. DeTar, S. Datta, S. Gottlieb, U. M. Heller, and R. Sugar, Phys. Rev. D 64, 054506 (2001).

[64] C. Aubin, C. Bernard, C. DeTar, J. Osborn, S. Gottlieb, E. B. Gregory, D. Toussaint, U. M. Heller, J. E. Hetrick, and R. Sugar, Phys. Rev. D 70, 094505 (2004).

[65] A. Bazavov et al. (MILC Collaboration), Rev. Mod. Phys. 82, 1349 (2010).

[66] D. J. Wilson, R. A. Briceno, J. J. Dudek, R. G. Edwards, and C. E. Thomas, Phys. Rev. D 92, 094502 (2015).

[67] J. Gasser and H. Leutwyler, Nucl. Phys. B250, 465 (1985).

[68] G. Herdoiza, K. Jansen, C. Michael, K. Ottnad, and C. Urbach, J. High Energy Phys. 05 (2013) 038.

[69] R. Berlich, S. Gabriel, A. Garcia, and M. Kunze, Data Driven e-Science, Conference Proceedings of ISGC 2010 (Springer, New York, 2010), p. 303.

[70] Y.-R. Liu, X. Liu, and S.-L. Zhu, Phys. Rev. D 79, 094026 (2009).

[71] F.-K. Guo and U.-G. Meißner, Phys. Rev. D 84, 014013 (2011).

[72] M. F. M. Lutz and E. E. Kolomeitsev, Nucl. Phys. A730, 392 (2004).

[73] M. F. M. Lutz and I. Vidana, Eur. Phys. J. A 48, 124 (2012).

[74] M. F. M. Lutz, E. E. Kolomeitsev, and C. L. Korpa, Phys. Rev. D 92, 016003 (2015).

[75] M. Lüscher, Nucl. Phys. B354, 531 (1991).

[76] M. Lüscher, Nucl. Phys. B364, 237 (1991).

[77] D.-L. Yao, M.-L. Du, F.-K. Guo, and U.-G. Meißner, J. High Energy Phys. 11 (2015) 058.

[78] M.-L. Du, F.-K. Guo, U.-G. Meißner, and D.-L. Yao, Eur. Phys. J. C 77, 728 (2017).

[79] F.-K. Guo, C. Hanhart, U.-G. Meißner, Q. Wang, Q. Zhao, and B.-S. Zou, Rev. Mod. Phys. 90, 015004 (2018).

[80] R. Aaij et al. (LHCb Collaboration), Phys. Rev. D 90, 072003 (2014).

[81] R. Aaij et al. (LHCb Collaboration), Phys. Rev. D 94, 072001 (2016).

[82] A. Faessler, T. Gutsche, V. E. Lyubovitskij, and Y.-L. Ma, Phys. Rev. D 76, 014005 (2007).

[83] M.-L. Du, M. Albaladejo, P. Fernndez-Soler, F.-K. Guo, C. Hanhart, U.-G. Meißner, J. Nieves, and D.-L. Yao, arXiv: 1712.07957.

[84] P. Colangelo and F. De Fazio, Phys. Lett. B 570, 180 (2003).

[85] M. F. M. Lutz et al. (PANDA Collaboration), arXiv:0903 .3905 . 